\title{
Asymmetric Synthesis of 3,4-Dihydrocoumarins by Rhodium-Catalyzed Reaction of 3-(2-Hydroxyphenyl)cyclobutanones
}

\author{
Takanori Matsuda, Masanori Shigeno, and Masahiro Murakami* \\ Department of Synthetic Chemistry and Biological Chemistry, \\ Kyoto University, Katsura, Kyoto 615-8510, Japan
}

\section{Supporting Information}

General. All reactions were carried out with standard Schlenk techniques under an argon or nitrogen atmosphere. Column chromatography was carried out on Wakogel C-200 (75-150 $\mu \mathrm{m})$. Preparative thin-layer chromatography was performed on silica gel $60 \mathrm{PF}_{254}$ (Merck). ${ }^{1} \mathrm{H}$ and ${ }^{13} \mathrm{C}$ NMR data were taken in $\mathrm{CDCl}_{3}$ at 300.07 or $400.44 \mathrm{MHz}$ and $75.46 \mathrm{MHz}$, respectively. Proton chemical shifts were referenced to the residual proton signal of the solvent at $7.26 \mathrm{ppm}$. Carbon chemical shifts were referenced to the carbon signal of the solvent at $77.0 \mathrm{ppm}$.

Materials. Dimethyltitanocene ${ }^{1}$ and $[\mathrm{Rh}(\mathrm{OH})(\mathrm{cod})]_{2}{ }^{2}$ were prepared according to the literature procedures. $(R)$-SEGPHOS was obtained from Takasago International Corporation. All other commercially available chemical resources were used as received without further purification.

\section{Preparation of Cyclobutanones 1}

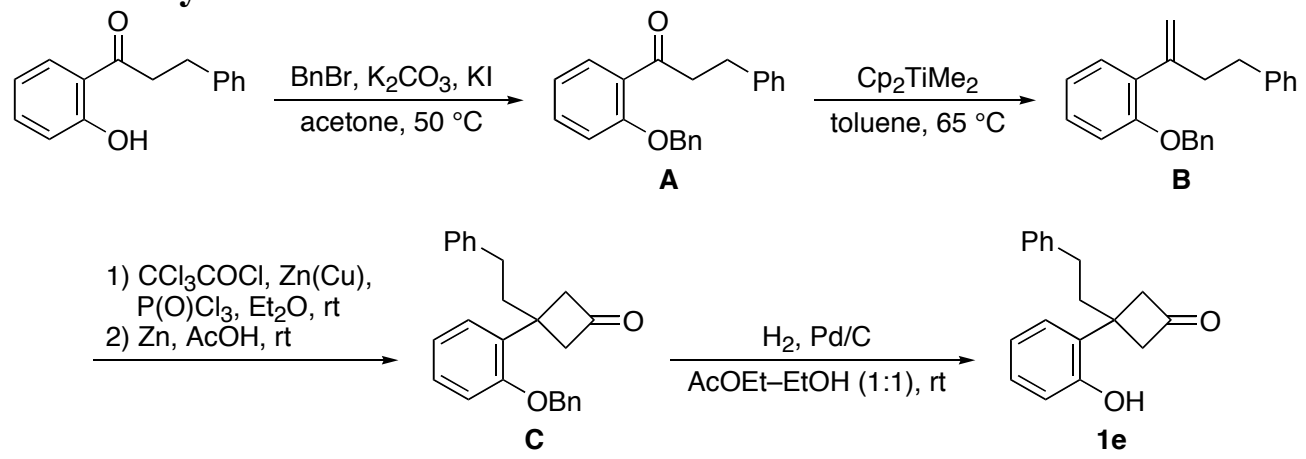

1-[2-(Benzyloxy)phenyl]-3-phenylpropan-1-one (A). To a suspension of 1-(2-hydroxyphenyl)-3-phenylpropan-1-one (11.3 g, $50.0 \mathrm{mmol}), \mathrm{K}_{2} \mathrm{CO}_{3}(13.8 \mathrm{~g}, 100 \mathrm{mmol})$ and $\mathrm{KI}(8.30 \mathrm{~g}, 50 \mathrm{mmol})$ in acetone $(200 \mathrm{~mL})$ was added benzyl bromide $(22.0 \mathrm{~g}, 129 \mathrm{mmol})$. After stirring at $50{ }^{\circ} \mathrm{C}$ for $11 \mathrm{~h}$, the reaction mixture filtered, and the filtrate was evaporated. The residue was purified by column chromatography on silica gel (hexane:AcOEt $=8: 1$ ) to afford $\mathbf{A}$ (12.6 g, $39.8 \mathrm{mmol}, 80 \%):{ }^{1} \mathrm{H}$ NMR (400 MHz) $\delta 2.96-3.01(\mathrm{~m}, 2 \mathrm{H}), 3.30-3.35(\mathrm{~m}, 2 \mathrm{H}), 5.15$ (s,

(1) Ndakala, A. J.; Hashemzadeh, M.; So, R. C.; Howell, A. R. Org. Lett. 2002, 4, 1719.

(2) Uson, R.; Oro, L. A.; Cabeza, J. A. Inorg. Synth. 1985, 23, 126. 
2H), 7.00-7.08 (m, 4H), 7.14-7.19 (m, 1H), 7.19-7.27 (m, 2H), 7.33-7.48 (m, 6H), $7.72(\mathrm{dd}, J=$ $5.7,1.5 \mathrm{~Hz}, 1 \mathrm{H}) ;{ }^{13} \mathrm{C}$ NMR $\delta 30.2,45.5,70.7,112.6,120.9,125.7,127.6,128.17,128.22,128.5$, $128.6,130.4,133.3,135.9,141.4,157.6,201.5$ [one $\mathrm{sp}^{2}$ carbon signal is missing due to overlapping]; HRMS (FAB) calcd for $\mathrm{C}_{22} \mathrm{H}_{21} \mathrm{O}_{2}\left(\mathrm{M}^{+}+\mathrm{H}\right) 317.1542$, found 317.1544.

1-(Benzyloxy)-2-(4-phenylbut-1-en-2-yl)benzene (B). To a solution of A $\mathbf{A} 6.32 \mathrm{~g}, 20.0 \mathrm{mmol})$ in toluene $(100 \mathrm{~mL})$ was added dropwise a solution of $\mathrm{Cp}_{2} \mathrm{TiMe}_{2}(5.41 \mathrm{~g}, 26.0 \mathrm{mmol})$ in toluene $(100 \mathrm{~mL})$. After stirring at $65^{\circ} \mathrm{C}$ for $42 \mathrm{~h}$ in the dark, the mixture was poured into hexane. The resulting orange precipitate was filtered off, and the filtrate was evaporated. The residue was purified by column chromatography on silica gel (hexane: $\left.\mathrm{Et}_{2} \mathrm{O}=40: 1\right)$ to give $\mathbf{B}(4.40 \mathrm{~g}, 14.0$ mmol, 70\%): ${ }^{1} \mathrm{H}$ NMR $(300 \mathrm{MHz}) \delta 2.68(\mathrm{dd}, J=10.1,6.2 \mathrm{~Hz}, 2 \mathrm{H}), 2.85(\mathrm{dd}, J=9.5,6.2 \mathrm{~Hz}$, $2 \mathrm{H}), 5.07(\mathrm{~d}, J=1.5 \mathrm{~Hz}, 1 \mathrm{H}), 5.10(\mathrm{~s}, 2 \mathrm{H}), 5.15(\mathrm{~d}, J=1.5 \mathrm{~Hz}, 1 \mathrm{H}), 6.92-6.99(\mathrm{~m}, 2 \mathrm{H})$, 7.07-7.14 (m, 2H), 7.14-7.28 (m, 5H), 7.28-7.45 (m, 5H); ${ }^{13} \mathrm{C}$ NMR $\delta 34.6,38.0,70.2,112.2$, 114.6, 120.8, 125.5, 127.2, 127.7, 128.1, 128.4 [overlapping], 128.5, 130.4, 132.3, 137.1, 142.2, 148.3, 155.6; HRMS (FAB) calcd for $\mathrm{C}_{23} \mathrm{H}_{22} \mathrm{O}\left(\mathrm{M}^{+}\right) 314.1671$, found 314.1667.

3-[2-(Benzyloxy)phenyl]-3-phenethylcyclobutanone (C). To a mixture of zinc-copper couple $(1.66 \mathrm{~g}, 25.4 \mathrm{mmol})$ and $\mathbf{B}(4.40 \mathrm{~g}, 14.0 \mathrm{mmol})$ in $\mathrm{Et}_{2} \mathrm{O}(20 \mathrm{~mL})$ was added dropwise a solution of $\mathrm{CCl}_{3} \mathrm{COCl}(4.07 \mathrm{~g}, 22.4 \mathrm{mmol})$ and $\mathrm{P}(\mathrm{O}) \mathrm{Cl}_{3}(3.43 \mathrm{~g}, 22.4 \mathrm{mmol})$ in $\mathrm{Et}_{2} \mathrm{O}(20 \mathrm{~mL})$ over $15 \mathrm{~min}$. After stirring at room temperature for $21 \mathrm{~h}$, the reaction mixture was filtered $\left(\right.$ Celite $\left.{ }^{\circledR}, \mathrm{Et}_{2} \mathrm{O}\right)$. The filtrate was washed with saturated $\mathrm{NaHCO}_{3}$ aqueous solution and brine, dried over $\mathrm{MgSO}_{4}$, and concentrated to afford dichlorocyclobutanone. To a suspension of zinc $(7.32 \mathrm{~g}, 112 \mathrm{mmol})$ in $\mathrm{AcOH}(20 \mathrm{~mL})$ was added dropwise the crude dichlorocyclobutanone in $\mathrm{AcOH}(20 \mathrm{~mL})$. After stirring at room temperature for $6 \mathrm{~h}$, the reaction mixture was filtered $\left(\right.$ Celite ${ }^{\circledR}, \mathrm{Et}_{2} \mathrm{O}$ ). The filtrate was washed with water, saturated $\mathrm{NaHCO}_{3}$ aqueous solution, and brine. The organic phase was dried over $\mathrm{MgSO}_{4}$ and concentrated. The residue was purified by column chromatography on silica gel (hexane:AcOEt $=4: 1)$ to afford cyclobutanone $\mathbf{C}(4.10 \mathrm{~g}$, $11.5 \mathrm{mmol}, 82 \%):{ }^{1} \mathrm{H}$ NMR $(300 \mathrm{MHz}) \delta 2.15-2.24(\mathrm{~m}, 2 \mathrm{H}), 2.33-2.42(\mathrm{~m}, 2 \mathrm{H}), 3.08-3.18(\mathrm{~m}$, $2 \mathrm{H}), 3.41-3.52(\mathrm{~m}, 2 \mathrm{H}), 5.10(\mathrm{~s}, 2 \mathrm{H}), 6.98-7.07(\mathrm{~m}, 4 \mathrm{H}), 7.11-7.41(\mathrm{~m}, 10 \mathrm{H}) ;{ }^{13} \mathrm{C}$ NMR $\delta 32.3$, $36.5,41.4,57.7,70.0,111.9,120.3,125.7,127.4,128.0,128.2$ [overlapping], 128.3, 128.6, 128.8, 132.8, 136.6, 141.7, 156.4, 208.6; HRMS (FAB) calcd for $\mathrm{C}_{25} \mathrm{H}_{25} \mathrm{O}_{2}\left(\mathrm{M}^{+}+\mathrm{H}\right) 357.1855$, found 357.1861 .

3-(2-Hydroxyphenyl)-3-phenethylcyclobutanone (1e). A mixture of $\mathbf{C}(4.00 \mathrm{~g}, 11.2 \mathrm{mmol})$ and $\mathrm{Pd} / \mathrm{C}(5 \%, 2.00 \mathrm{~g})$ in $\mathrm{MeOH}-\mathrm{AcOEt}(1: 1,30 \mathrm{~mL})$ was stirred under hydrogen atmosphere at room temperature for $17 \mathrm{~h}$. The reaction mixture was filtered $\left(\mathrm{Celite}{ }^{\circledR}, \mathrm{AcOEt}\right)$ and concentrated. The residue was purified by column chromatography on silica gel (hexane:AcOEt $=3: 1$ ) to afford 1e (2.96 g, $11.1 \mathrm{mmol}$, 99\%), which exists as an equilibrium mixture with the corresponding hemiketal (cyclobutanone:hemiketal $=89: 11$ by ${ }^{1} \mathrm{H}$ NMR). ${ }^{1} \mathrm{H}$ NMR $(300 \mathrm{MHz}) \delta$ 2.16-2.27 (m, 2H), 2.35-2.45 (m, 2H), 3.13-3.24 (m, 2H), 3.43-3.55 (m, 2H), $4.81(\mathrm{~s}, 1 \mathrm{H}), 6.76$ (dd, $J=7.8,0.6 \mathrm{~Hz}, 1 \mathrm{H}), 6.97(\mathrm{dt}, J=1.2,7.5 \mathrm{~Hz}, 1 \mathrm{H}), 7.03-7.09(\mathrm{~m}, 2 \mathrm{H}), 7.11-7.26(\mathrm{~m}, 5 \mathrm{H})$; ${ }^{13}$ C NMR $\delta 32.2,36.3,41.4,57.4,70.0,115.9,120.4,125.7,128.2,128.3,129.0,130.7,141.7$, 153.6, 209.6 [one $\mathrm{sp}^{2}$ carbon signal is missing due to overlapping]; HRMS (EI) calcd for 
$\mathrm{C}_{18} \mathrm{H}_{18} \mathrm{O}_{2}\left(\mathrm{M}^{+}\right)$266.1307, found 266.1299.

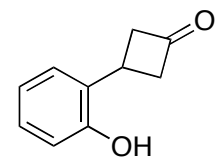

3-(2-Hydroxyphenyl)cyclobutanone (1a). ${ }^{3}{ }^{1} \mathrm{H}$ NMR $(300 \mathrm{MHz}) \delta 3.31-3.52(\mathrm{~m}, 4 \mathrm{H}), 3.75$ (quint, $J=8.3 \mathrm{~Hz}, 1 \mathrm{H}), 5.12(\mathrm{~s}, 1 \mathrm{H}), 6.77(\mathrm{dd}, J=7.7,1.1 \mathrm{~Hz}, 1 \mathrm{H}), 6.94(\mathrm{dt}, J=1.1,7.5 \mathrm{~Hz}, 1 \mathrm{H})$, $7.15(\mathrm{dt}, J=1.6,7.6 \mathrm{~Hz}, 1 \mathrm{H}), 7.22(\mathrm{dd}, J=7.8,1.5 \mathrm{~Hz}, 1 \mathrm{H}) ;{ }^{13} \mathrm{C}$ NMR $\delta 25.2,52.8,115.4,120.3$, 127.8 [overlapping], 128.8, 154.2, 211.1; HRMS (EI) calcd for $\mathrm{C}_{10} \mathrm{H}_{10} \mathrm{O}_{2}\left(\mathrm{M}^{+}\right)$162.0681, found 162.0685 .

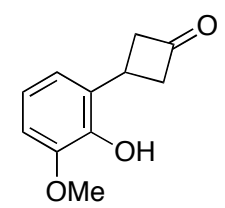

3-(2-Hydroxy-3-methoxyphenyl)cyclobutanone (1b). ${ }^{1} \mathrm{H}$ NMR (300 MHz) $\delta 3.31-3.50(\mathrm{~m}$, $4 \mathrm{H}$ ), 3.77 (quint, $J=8.2 \mathrm{~Hz}, 1 \mathrm{H}), 3.90(\mathrm{~s}, 3 \mathrm{H}), 5.83(\mathrm{~s}, 1 \mathrm{H}), 6.77-6.88(\mathrm{~m}, 3 \mathrm{H}) ;{ }^{13} \mathrm{C}$ NMR $\delta 24.6$, 53.0, 56.0, 109.1, 119.4, 119.6, 128.5, 143.6, 146.4, 208.3; HRMS (EI) calcd for $\mathrm{C}_{11} \mathrm{H}_{12} \mathrm{O}_{3}\left(\mathrm{M}^{+}\right)$ 192.0786, found 192.0785 .<smiles>O=C1CC(c2cc(Cl)ccc2O)C1</smiles>

3-(5-Chloro-2-hydroxyphenyl)cyclobutanone (1c). ${ }^{4}{ }^{1} \mathrm{H}$ NMR $(300 \mathrm{MHz}) \delta 3.28-3.51$ (m, $4 \mathrm{H}$ ), 3.69 (quint, $J=8.2 \mathrm{~Hz}, 1 \mathrm{H}), 6.63(\mathrm{~s}, 1 \mathrm{H}), 6.75(\mathrm{~d}, J=8.5 \mathrm{~Hz}, 1 \mathrm{H}), 7.07$ (dd, $J=8.5,2.6 \mathrm{~Hz}$, $1 \mathrm{H}), 7.15(\mathrm{~d}, J=2.6 \mathrm{~Hz}, 1 \mathrm{H}) ;{ }^{13} \mathrm{C}$ NMR $\delta 25.3,52.7,116.6,124.9,127.5,127.9,130.5,152.8$, 210.6; HRMS (EI) calcd for $\mathrm{C}_{10} \mathrm{H}_{9} \mathrm{ClO}_{2}\left(\mathrm{M}^{+}\right)$196.0291, found 196.0293 .

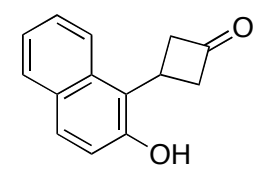

3-(2-Hydroxynaphthalen-1-yl)cyclobutanone (1d). The title compound was isolated as a mixture with the corresponding hemiketal (cyclobutanone:hemiketal = 86:14). ${ }^{1} \mathrm{H}$ NMR $(300$ MHz) $\delta 3.46-3.60(\mathrm{~m}, 2 \mathrm{H}), 3.72-3.84(\mathrm{~m}, 2 \mathrm{H}), 4.78(\mathrm{tt}, J=9.8,6.6 \mathrm{~Hz}, 1 \mathrm{H}), 5.77(\mathrm{~s}, 1 \mathrm{H}), 7.08(\mathrm{~d}$, $J=8.7 \mathrm{~Hz}, 1 \mathrm{H}), 7.36(\mathrm{t}, J=7.5 \mathrm{~Hz}, 1 \mathrm{H}), 7.48-7.55(\mathrm{~m}, 1 \mathrm{H}), 7.68(\mathrm{~d}, J=8.7 \mathrm{~Hz}, 1 \mathrm{H}), 7.80(\mathrm{~d}, J=$ $8.1 \mathrm{~Hz}, 1 \mathrm{H}), 8.11(\mathrm{~d}, J=8.4 \mathrm{~Hz}, 1 \mathrm{H}) ;{ }^{13} \mathrm{C}$ NMR $\delta 21.7,53.3,118.5,120.8,122.6,123.4,127.0$, 128.8, 129.1, 129.5, 133.4, 151.8, 211.3; HRMS (EI) calcd for $\mathrm{C}_{14} \mathrm{H}_{12} \mathrm{O}_{2}\left(\mathrm{M}^{+}\right)$212.0837, found 212.0842 .

(3) [294669-29-7].

(4) [294669-31-1]. 
<smiles>COc1ccc(O)c(C2(CCc3ccccc3)CC(=O)C2)c1</smiles>

3-(2-Hydroxy-5-methoxyphenyl)-3-phenethylcyclobutanone (1f). The title compound was isolated as a mixture with the corresponding hemiketal (cyclobutanone:hemiketal $=90: 10$ ). ${ }^{1} \mathrm{H}$ NMR $(300 \mathrm{MHz}) \delta 2.16-2.25(\mathrm{~m}, 2 \mathrm{H}), 2.38-2.46(\mathrm{~m}, 2 \mathrm{H}), 3.12-3.23(\mathrm{~m}, 2 \mathrm{H}), 3.42-3.53(\mathrm{~m}, 2 \mathrm{H})$, 3.79 (s, 3H), 4.56 (br s, 1H), 6.69-6.73 (m, 3H), 7.05-7.18 (m, 3H), 7.19-7.27 (m, 2H); ${ }^{13} \mathrm{C} \mathrm{NMR}$ $\delta 32.2,36.4,41.4,55.8,57.4,112.0,115.6,116.4,125.8,128.2,128.3,132.1,141.6,147.5,153.2$, 209.0; HRMS (EI) calcd for $\mathrm{C}_{19} \mathrm{H}_{20} \mathrm{O}_{3}\left(\mathrm{M}^{+}\right)$296.1412, found 296.1418.<smiles>CCC1(c2ccccc2O)CC(=O)C1</smiles>

3-Ethyl-3-(2-hydroxyphenyl)cyclobutanone (1g). The title compound was isolated as a mixture with the corresponding hemiketal (cyclobutanone:hemiketal $=89: 11)$. ${ }^{1} \mathrm{H}$ NMR $(300$ MHz) $\delta 0.75(\mathrm{t}, J=7.4 \mathrm{~Hz}, 3 \mathrm{H}), 1.92(\mathrm{q}, J=7.3 \mathrm{~Hz}, 2 \mathrm{H}), 3.07-3.19(\mathrm{~m}, 2 \mathrm{H}), 3.37-3.49(\mathrm{~m}, 2 \mathrm{H})$, $5.25(\mathrm{~s}, 1 \mathrm{H}), 6.75(\mathrm{~d}, J=8.1 \mathrm{~Hz}, 1 \mathrm{H}), 6.92(\mathrm{dt}, J=1.0,7.5 \mathrm{~Hz}, 1 \mathrm{H}), 7.07(\mathrm{dd}, J=7.5,1.5 \mathrm{~Hz}$, $1 \mathrm{H}), 7.14$ (dt, $J=1.7,7.5 \mathrm{~Hz}, 1 \mathrm{H}) ;{ }^{13} \mathrm{C} \mathrm{NMR} \delta 10.0,32.3,36.7,56.9,115.8,120.1,127.9,129.1$, 130.9, 153.7, 210.9; HRMS (EI) calcd for $\mathrm{C}_{12} \mathrm{H}_{14} \mathrm{O}_{2}\left(\mathrm{M}^{+}\right)$190.0994, found 190.0997.<smiles>O=C1CCCC1c1ccccc1O</smiles>

3-(2-Hydroxyphenyl)-3-isopropylcyclobutanone (1h). The title compound was isolated as a mixture with the corresponding hemiketal (cyclobutanone:hemiketal $=84: 16) .{ }^{1} \mathrm{H}$ NMR $(300$ MHz) $\delta 0.88(\mathrm{~d}, J=6.6 \mathrm{~Hz}, 6 \mathrm{H}), 2.17(\mathrm{sext}, J=6.7 \mathrm{~Hz}, 1 \mathrm{H}), 3.23-3.33(\mathrm{~m}, 2 \mathrm{H}), 3.34-3.45(\mathrm{~m}$, $2 \mathrm{H}), 4.88(\mathrm{~s}, 1 \mathrm{H}), 6.73(\mathrm{~d}, J=7.8 \mathrm{~Hz}, 1 \mathrm{H}), 6.92(\mathrm{dt}, J=1.2,7.5 \mathrm{~Hz}, 1 \mathrm{H}), 7.05(\mathrm{dd}, J=7.8,1.5$ $\mathrm{Hz}, 1 \mathrm{H}), 7.14(\mathrm{dt}, J=1.4,7.4 \mathrm{~Hz}, 1 \mathrm{H}) ;{ }^{13} \mathrm{C}$ NMR $\delta 18.2,35.5,40.0,55.3,116.1,119.7,127.9$, 130.2, 130.4, 154.1, 212.2; HRMS (EI) calcd for $\mathrm{C}_{13} \mathrm{H}_{16} \mathrm{O}_{2}\left(\mathrm{M}^{+}\right)$204.1150, found 204.1149.

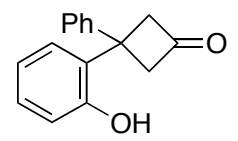

3-(2-Hydroxyphenyl)-3-phenylcyclobutanone (1i). The title compound was isolated as a mixture with the corresponding hemiketal (cyclobutanone:hemiketal = 29:71). ${ }^{1} \mathrm{H}$ NMR $(300$ MHz) $\delta 3.67-3.95(\mathrm{~m}, 4 \mathrm{H}), 5.02(\mathrm{~s}, 1 \mathrm{H}), 6.76(\mathrm{dd}, J=8.1,0.9 \mathrm{~Hz}, 1 \mathrm{H}), 6.99(\mathrm{~d}, J=7.8 \mathrm{~Hz}, 1 \mathrm{H})$, 7.09-7.48 (m, 7H); ${ }^{13} \mathrm{C}$ NMR $\delta 39.8,42.1,42.6,59.7,99.8,114.3,116.5,119.8,120.8,124.2$, 126.4, 127.0, 127.5, 128.4, 128.48, 128.52, 128.6, 132.3, 135.6, 141.4, 146.0, 153.3, 153.5, 208.0 [The spectra of 1i was indistinguishable from that of the hemiketal]; HRMS (EI) calcd for $\mathrm{C}_{16} \mathrm{H}_{14} \mathrm{O}_{2}\left(\mathrm{M}^{+}\right)$238.0994, found 238.0997. 
<smiles>Cc1ccc(O)c(C2(CCCO)CC(=O)C2)c1</smiles>

3-(2-Hydroxy-5-methylphenyl)-3-(3-hydroxypropyl)cyclobutanone $\quad(\mathbf{1 j}) . \quad$ The title compound was isolated as a mixture with the corresponding hemiketal (cyclobutanone:hemiketal $=79: 21) .{ }^{1} \mathrm{H}$ NMR $(300 \mathrm{MHz}) \delta 1.32-1.45(\mathrm{~m}, 2 \mathrm{H}+1 \mathrm{H}), 1.90-1.97(\mathrm{~m}, 2 \mathrm{H}), 2.28(\mathrm{~s}, 3 \mathrm{H})$, 3.07-3.18 (m, 2H), 3.39-3.49 (m, 2H), $3.56(\mathrm{t}, J=6.5 \mathrm{~Hz}, 2 \mathrm{H}), 5.09(\mathrm{~s}, 1 \mathrm{H}), 6.62(\mathrm{~d}, J=7.8 \mathrm{~Hz}$, $1 \mathrm{H}), 6.86(\mathrm{~d}, J=1.8 \mathrm{~Hz}, 1 \mathrm{H}), 6.89-6.95(\mathrm{~m}, 1 \mathrm{H}) ;{ }^{13} \mathrm{C}$ NMR $\delta 20.6,29.0,35.8,36.1,57.4,62.9$, $115.9,128.4,129.4,130.8,151.4,209.2$ [one $\mathrm{sp}^{2}$ carbon signal could not be assigned due to overlap with signals of the hemiketal]; HRMS (CI) calcd for $\mathrm{C}_{14} \mathrm{H}_{18} \mathrm{O}_{3}\left(\mathrm{M}^{+}\right)$234.1256, found 234.1256.

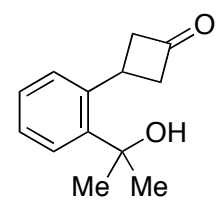

3-[2-(2-Hydroxypropan-2-yl)phenyl]cyclobutanone (7). ${ }^{1} \mathrm{H}$ NMR (300 MHz) $\delta 1.70(\mathrm{~s}, 6 \mathrm{H})$, $2.03(\mathrm{~s}, 1 \mathrm{H}), 3.13-3.26(\mathrm{~m}, 2 \mathrm{H}), 3.41-3.54(\mathrm{~m}, 2 \mathrm{H}), 4.81(\mathrm{ddd}, J=16.5,9.2,7.5 \mathrm{~Hz}, 1 \mathrm{H})$, 7.15-7.23 (m, 1H), 7.29-7.36 (m, 1H), $7.39(\mathrm{dd}, J=7.8,1.2 \mathrm{~Hz}, 1 \mathrm{H}), 7.49(\mathrm{~d}, J=7.8 \mathrm{~Hz}, 1 \mathrm{H})$; ${ }^{13} \mathrm{C}$ NMR $\delta 24.8,31.8,56.6,125.2,126.1,126.4,128.0,143.1,145.3,208.5$; HRMS (CI) calcd for $\mathrm{C}_{13} \mathrm{H}_{17} \mathrm{O}_{2}\left(\mathrm{M}^{+}+\mathrm{H}\right)$ 205.1229, found 205.1229.

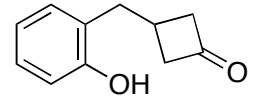

3-(2-Hydroxybenzyl)cyclobutanone (9). ${ }^{1} \mathrm{H}$ NMR $(300 \mathrm{MHz}) \delta$ 2.74-2.95 (m, 5H), 3.06-3.20 $(\mathrm{m}, 2 \mathrm{H}), 5.07(\mathrm{~s}, 1 \mathrm{H}), 6.73-6.78(\mathrm{~m}, 1 \mathrm{H}), 6.89(\mathrm{dt}, J=1.1,7.4 \mathrm{~Hz}, 1 \mathrm{H}), 7.07-7.15(\mathrm{~m}, 2 \mathrm{H}) ;{ }^{13} \mathrm{C}$ NMR $\delta 23.9,35.8,52.1,115.2,120.5,126.2,127.6,130.4,153.8,210.9$; HRMS (EI) calcd for $\mathrm{C}_{11} \mathrm{H}_{12} \mathrm{O}_{2}\left(\mathrm{M}^{+}\right)$176.0837, found 176.0838.

General Procedure for Rhodium-Catalyzed Enantioselective Addition/Ring-Opening of Cyclobutanones 1 (Scheme 1, Chart 1, and Table 1)

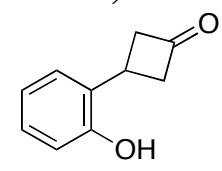

$1 a$

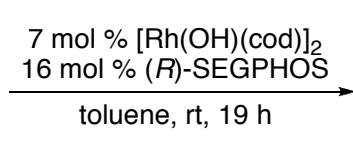
toluene, rt, $19 \mathrm{~h}$

2

(S)-4-Methylchroman-2-one (2a). ${ }^{5}$ To a mixture of $[\mathrm{Rh}(\mathrm{OH})(\mathrm{cod})]_{2}(6.39 \mathrm{mg}, 14.0 \mu \mathrm{mol}, 7.0$ mol \%) and $(R)$-SEGPHOS (19.6 mg, $32.1 \mu \mathrm{mol}, 16 \mathrm{~mol} \%)$ in toluene (1.6 mL) was added 1a $(32.4 \mathrm{mg}, 0.200 \mathrm{mmol})$ in toluene $(0.4 \mathrm{~mL})$, and the reaction mixture was stirred for $19 \mathrm{~h}$ at room

(5) $[30825-54-8]$. 
temperature. The reaction mixture was filtered through a pad of Florisil ${ }^{\circledR}\left(\mathrm{Et}_{2} \mathrm{O}-\mathrm{AcOEt}\right)$ and concentrated. The residue was purified by preparative thin-layer chromatography of silica gel (hexane:AcOEt $=8: 1)$ to afford $2 \mathbf{a}(25.1 \mathrm{mg}, 0.155 \mathrm{mmol}, 77 \%)$ : $[\alpha]_{\mathrm{D}}^{23}-22.1\left(c 0.86, \mathrm{CHCl}_{3}\right)$; ${ }^{1} \mathrm{H}$ NMR $(300 \mathrm{MHz}) \delta 1.34(\mathrm{~d}, J=7.2 \mathrm{~Hz}, 3 \mathrm{H}), 2.58(\mathrm{dd}, J=15.7,7.1 \mathrm{~Hz}, 1 \mathrm{H}), 2.85(\mathrm{dd}, J=$ 15.7, $5.4 \mathrm{~Hz}, 1 \mathrm{H}), 3.18(\mathrm{sext}, J=6.7 \mathrm{~Hz}, 1 \mathrm{H}), 7.06(\mathrm{~d}, J=8.1 \mathrm{~Hz}, 1 \mathrm{H}), 7.13(\mathrm{dt}, J=1.1,7.4 \mathrm{~Hz}$, $1 \mathrm{H}), 7.20-7.30(\mathrm{~m}, 2 \mathrm{H}) ;{ }^{13} \mathrm{C}$ NMR $\delta 19.9,29.5,36.8,116.9,124.5,126.4,127.8,128.2,151.1$, 168.3; IR (neat) 1786, 1717, 1605, 1462, 1287, 1237, $764 \mathrm{~cm}^{-1}$; HRMS (EI) calcd for $\mathrm{C}_{10} \mathrm{H}_{10} \mathrm{O}_{2}$ $\left(\mathrm{M}^{+}\right)$162.0681, found 162.0682.

The enantiomeric excess was determined to be $>99 \%$ by chiral HPLC [Daicel CHIRALCEL ${ }^{\circledR}$ OD-H column, hexane: $i-\mathrm{PrOH}=98: 2,0.6 \mathrm{~mL} / \mathrm{min}$, retention times: $t_{1}=18.5 \mathrm{~min}(S) ; t_{2}=19.5$ $\min (R)$.].

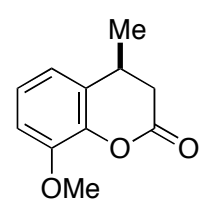

8-Methoxy-4-methylchroman-2-one (2b). According to the general procedure, but using 3.5 mol \% $[\mathrm{Rh}(\mathrm{OH})(\mathrm{cod})]_{2}$ and $8.0 \mathrm{~mol} \%(R)-\mathrm{SEGPHOS}, \mathbf{2 b}(30.2 \mathrm{mg}, 0.157 \mathrm{mmol}$, 79\%) was obtained from $1 \mathrm{~b}(38.4 \mathrm{mg}, 0.200 \mathrm{mmol}):[\alpha]^{23}{ }_{\mathrm{D}}-21.4\left(c 1.5, \mathrm{CHCl}_{3}\right) ;{ }^{1} \mathrm{H} \mathrm{NMR}(300 \mathrm{MHz}) \delta$ $1.31(\mathrm{~d}, J=6.9 \mathrm{~Hz}, 3 \mathrm{H}), 2.58(\mathrm{dd}, J=15.9,6.6 \mathrm{~Hz}, 1 \mathrm{H}), 2.82(\mathrm{dd}, J=15.8,5.3 \mathrm{~Hz}, 1 \mathrm{H}), 3.16$ (sext, $J=6.7 \mathrm{~Hz}, 1 \mathrm{H}), 3.87(\mathrm{~s}, 3 \mathrm{H}), 6.80(\mathrm{dt}, J=7.5,0.6 \mathrm{~Hz}, 1 \mathrm{H}), 6.85(\mathrm{~d}, J=8.1 \mathrm{~Hz}, 1 \mathrm{H}), 7.06$ $(\mathrm{t}, J=8.1 \mathrm{~Hz}, 1 \mathrm{H}) ;{ }^{13} \mathrm{C}$ NMR $\delta 19.9,29.8,36.6,56.0,110.9,117.9,124.4,128.9,140.3,147.5$, 167.6; HRMS (EI) calcd for $\mathrm{C}_{11} \mathrm{H}_{12} \mathrm{O}_{3}\left(\mathrm{M}^{+}\right)$192.0786, found 192.0782.

The enantiomeric excess was determined to be $99 \%$ by chiral HPLC [Daicel CHIRALCEL ${ }^{\circledR}$ OD-H column, hexane: $i-\mathrm{PrOH}=97: 3,0.6 \mathrm{~mL} / \mathrm{min}$, retention times: $t_{1}=35.0 \mathrm{~min}$ (major); $t_{2}=$ 37.7 min (minor)].<smiles>[X]c1cc(Cl)ccc1OC(=O)CO</smiles>

6-Chloro-4-methylchroman-2-one (2c). According to the general procedure, but using 3.5 $\mathrm{mol} \%[\mathrm{Rh}(\mathrm{OH})(\mathrm{cod})]_{2}$ and $8.0 \mathrm{~mol} \%(R)-\mathrm{SEGPHOS}$ in toluene-THF (4:1), 2c (33.5 mg, 0.171 mmol, 85\%) was obtained from 1c $(39.3 \mathrm{mg}, 0.200 \mathrm{mmol}) .[\alpha]_{\mathrm{D}}^{21}-4.8\left(c 1.3, \mathrm{CHCl}_{3}\right) ;{ }^{1} \mathrm{H} \mathrm{NMR}$ $(300 \mathrm{MHz}) \delta 1.33(\mathrm{~d}, J=6.9 \mathrm{~Hz}, 3 \mathrm{H}), 2.56(\mathrm{dd}, J=15.9,7.5 \mathrm{~Hz}, 1 \mathrm{H}), 2.83(\mathrm{dd}, J=15.9,5.4 \mathrm{~Hz}$, $1 \mathrm{H}), 3.16$ (sext, $J=6.8 \mathrm{~Hz}, 1 \mathrm{H}), 6.98(\mathrm{dd}, J=5.6,2.6 \mathrm{~Hz}, 1 \mathrm{H}), 7.18-7.24(\mathrm{~m}, 2 \mathrm{H}) ;{ }^{13} \mathrm{C} \mathrm{NMR} \delta$ 19.6, 29.4, 36.3 118.3 , 126.4, 128.2, 129.4, 129.6, 149.7, 167.5; HRMS (EI) calcd for $\mathrm{C}_{10} \mathrm{H}_{9} \mathrm{ClO}_{2}$ $\left(\mathrm{M}^{+}\right)$196.0291, found 196.0299.

The enantiomeric excess was determined to be $98 \%$ by chiral HPLC [Daicel CHIRALCEL ${ }^{\circledR}$ AS-H column, hexane: $i-\mathrm{PrOH}=95: 5,0.6 \mathrm{~mL} / \mathrm{min}$, retention times: $t_{1}=25.2 \mathrm{~min}$ (minor); $t_{2}=$ 29.9 min (major)]. 


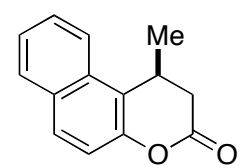

1,2-Dihydro-1-methylnaphtho[2,1-b]pyran-3-one (2d). According to the general procedure, but using $3.5 \mathrm{~mol} \%[\mathrm{Rh}(\mathrm{OH})(\mathrm{cod})]_{2}$ and $8.0 \mathrm{~mol} \%(R)-S E G P H O S$ in toluene-THF $(4: 1), 2 d$ (38.6 mg, $0.182 \mathrm{mmol}, 91 \%)$ was obtained from $1 \mathrm{~d}(42.4 \mathrm{mg}, 0.200 \mathrm{mmol}) .[\alpha]_{\mathrm{D}}^{21}+9.2(c 1.3$, $\left.\mathrm{CHCl}_{3}\right) ;{ }^{1} \mathrm{H}$ NMR $(300 \mathrm{MHz}) \delta 1.38(\mathrm{~d}, J=7.2 \mathrm{~Hz}, 3 \mathrm{H}), 2.87(\mathrm{dd}, J=15.8,2.4 \mathrm{~Hz}, 1 \mathrm{H}), 2.94(\mathrm{dd}$, $J=15.8,5.6 \mathrm{~Hz}, 1 \mathrm{H}), 3.78-3.90(\mathrm{~m}, 1 \mathrm{H}), 7.24(\mathrm{~d}, J=9.0 \mathrm{~Hz}, 1 \mathrm{H}), 7.48(\mathrm{ddd}, J=8.1,6.9,1.2 \mathrm{~Hz}$, $1 \mathrm{H}), 7.59$ (ddd, $J=8.5,6.8,1.4 \mathrm{~Hz}, 1 \mathrm{H}), 7.77(\mathrm{~d}, J=9.0 \mathrm{~Hz}, 1 \mathrm{H}), 7.86(\mathrm{dd}, J=7.8,0.6 \mathrm{~Hz}, 1 \mathrm{H})$, $7.93(\mathrm{~d}, J=8.4 \mathrm{~Hz}, 1 \mathrm{H}) ;{ }^{13} \mathrm{C}$ NMR $\delta 20.1,26.6,36.2,117.5,120.7,122.3,125.0,127.2,128.8$, 128.9, 130.3, 131.0, 148.4, 168.1; HRMS (EI) calcd for $\mathrm{C}_{14} \mathrm{H}_{12} \mathrm{O}_{2}\left(\mathrm{M}^{+}\right)$212.0837, found 212.0835 .

The enantiomeric excess was determined to be $98 \%$ by chiral HPLC [Daicel CHIRALCEL ${ }^{\circledR}$ AS-H column, hexane: $i-\mathrm{PrOH}=95: 5,0.6 \mathrm{~mL} / \mathrm{min}$, retention times: $t_{1}=24.8 \mathrm{~min}$ (minor); $t_{2}=$ $33.0 \mathrm{~min}$ (major)].

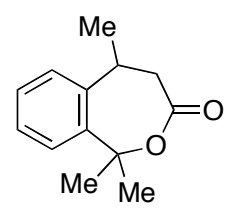

1,1,5-Trimethyl-4,5-dihydrobenzo[c]oxepin-3(1H)-one (8). To a mixture of $[\mathrm{Rh}(\mathrm{OH})(\mathrm{cod})]_{2}$ (6.39 mg, $14.0 \mu \mathrm{mol}, 7.0 \mathrm{~mol} \%$ ) and $(R)$-Tol-BINAP $(21.7 \mathrm{mg}, 32.0 \mu \mathrm{mol}, 16.0 \mathrm{~mol} \%)$ in toluene $(1.6 \mathrm{~mL})$ was added $7(40.9 \mathrm{mg}, 0.200 \mathrm{mmol})$ in toluene $(0.4 \mathrm{~mL})$, and the mixture was stirred for $12 \mathrm{~h}$ at $135{ }^{\circ} \mathrm{C}$. The reaction mixture was filtered through a pad of Florisil ${ }^{\circledR}$ $\left(\mathrm{Et}_{2} \mathrm{O}-\mathrm{AcOEt}\right)$, and the filtrate was concentrated. The residue was purified by preparative thin-layer chromatography of silica gel (hexane:AcOEt $=4: 1)$ to afford $\mathbf{8}(31.1 \mathrm{mg}, 0.152 \mathrm{mmol}$, 76\%): $[\alpha]^{20}{ }_{\mathrm{D}}-69.0\left(c 1.2, \mathrm{CHCl}_{3}\right) ;{ }^{1} \mathrm{H}$ NMR $(300 \mathrm{MHz}) \delta 1.41(\mathrm{~d}, J=6.9 \mathrm{~Hz}, 3 \mathrm{H}), 1.82(\mathrm{~s}, 3 \mathrm{H})$, $1.85(\mathrm{~s}, 3 \mathrm{H}), 2.84(\mathrm{dd}, J=15.6,6.3 \mathrm{~Hz}, 1 \mathrm{H}), 3.14-3.26(\mathrm{~m}, 2 \mathrm{H}), 7.13-7.24(\mathrm{~m}, 4 \mathrm{H}) ;{ }^{13} \mathrm{C}$ NMR $\delta$ 22.1, 32.3, 33.5, 35.1, 42.0, 84.6, 126.6, 127.1, 127.5, 130.4, 141.48, 141.53, 173.7; HRMS (EI) calcd for $\mathrm{C}_{13} \mathrm{H}_{16} \mathrm{O}_{2}\left(\mathrm{M}^{+}\right)$204.1150, found 204.1148.

The enantiomeric excess was determined to be $34 \%$ by chiral HPLC [Daicel CHIRALCEL ${ }^{\circledR}$ OD-H column, hexane: $i$-PrOH $=98: 2,0.6 \mathrm{~mL} / \mathrm{min}$, retention times: $t_{1}=19.4 \mathrm{~min}($ minor $) ; t_{2}=$ 23.0 min (major)].<smiles>CC1CC(=O)Oc2ccccc2C1</smiles>

4-Methyl-4,5-dihydrobenzo $[\boldsymbol{b}]$ oxepin-2(3H)-one (10). To a mixture of $[\mathrm{Rh}(\mathrm{OH})(\operatorname{cod})]_{2}(3.19$ $\mathrm{mg}, 7.0 \mu \mathrm{mol}, 3.5 \mathrm{~mol} \%$ ) and $(R)$-Tol-BINAP (10.9 mg, $16.1 \mu \mathrm{mol}, 8.1 \mathrm{~mol} \%)$ in toluene (1.6 $\mathrm{mL})$ was added $9(35.2 \mathrm{mg}, 0.200 \mathrm{mmol})$ in toluene $(0.4 \mathrm{~mL})$, and the mixture was stirred for 21 $\mathrm{h}$ at $110{ }^{\circ} \mathrm{C}$. The reaction mixture was filtered through a pad of Florisil ${ }^{\circledR}\left(\mathrm{Et}_{2} \mathrm{O}-\mathrm{AcOEt}\right)$, and the filtrate was concentrated. The residue was purified by preparative thin-layer chromatography of silica gel (hexane:AcOEt $=10: 1)$ to afford $10(21.5 \mathrm{mg}, 0.122 \mathrm{mmol}, 61 \%):[\alpha]^{21}{ }_{\mathrm{D}}-4.0(c 0.50$, 
$\left.\mathrm{CHCl}_{3}\right) ;{ }^{1} \mathrm{H}$ NMR $(400 \mathrm{MHz}) \delta 1.10(\mathrm{~d}, J=6.4 \mathrm{~Hz}, 3 \mathrm{H}), 2.09-2.17(\mathrm{~m}, 1 \mathrm{H}), 2.48(\mathrm{dd}, J=13.6$, $6.4 \mathrm{~Hz}, 1 \mathrm{H}), 2.52-2.64(\mathrm{~m}, 2 \mathrm{H}), 2.94-3.02(\mathrm{~m}, 1 \mathrm{H}), 7.09(\mathrm{dd}, J=8.0,0.8 \mathrm{~Hz}, 1 \mathrm{H}), 7.13-7.21(\mathrm{~m}$, 2H), 7.26-7.31 (m, 1H); ${ }^{13} \mathrm{C}$ NMR $\delta$ 20.4, 33.7, 36.1, 38.5, 119.2, 125.6, 128.3, 128.9, 130.2, 151.7, 170.7; HRMS (EI) calcd for $\mathrm{C}_{11} \mathrm{H}_{12} \mathrm{O}_{2}\left(\mathrm{M}^{+}\right)$176.0837, found 176.0840.

The enantiomeric excess was determined to be $28 \%$ by chiral HPLC [Daicel CHIRALCEL ${ }^{\circledR}$ OD-H column, hexane: $i$ PrOH $=98: 2,0.6 \mathrm{~mL} / \mathrm{min}$, retention times: $t_{1}=13.8$ min (major); $t_{2}=$ $18.2 \min ($ minor)].

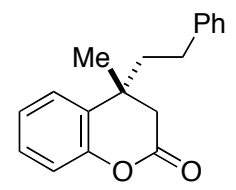

4-Methyl-4-phenethylchroman-2-one (2e). According to the general procedure, but using 3.5 mol \% $[\mathrm{Rh}(\mathrm{OH})(\mathrm{cod})]_{2}$ and $8.0 \mathrm{~mol} \%(R)$-Tol-BINAP, 2e (43.3 $\left.\mathrm{mg}, 0.163 \mathrm{mmol}, 81 \%\right)$ was prepared from 1e $(53.3 \mathrm{mg}, 0.200 \mathrm{mmol}) .[\alpha]_{\mathrm{D}}^{28}+39.0\left(c 1.7, \mathrm{CHCl}_{3}\right) ;{ }^{1} \mathrm{H} \mathrm{NMR}(300 \mathrm{MHz}) \delta$ $1.45(\mathrm{~s}, 3 \mathrm{H}), 1.85-1.97(\mathrm{~m}, 2 \mathrm{H}), 2.41-2.53(\mathrm{~m}, 1 \mathrm{H}), 2.55-2.70(\mathrm{~m}, 1 \mathrm{H}), 2.65(\mathrm{~d}, J=15.8 \mathrm{~Hz}, 1 \mathrm{H})$, $2.81(\mathrm{~d}, J=15.8 \mathrm{~Hz}, 1 \mathrm{H}), 7.06-7.13(\mathrm{~m}, 3 \mathrm{H}), 7.14-7.22(\mathrm{~m}, 2 \mathrm{H}), 7.22-7.36(\mathrm{~m}, 4 \mathrm{H}) ;{ }^{13} \mathrm{C} \mathrm{NMR} \delta$ 25.3, 30.6, 36.5, 41.7, 42.2, 117.3, 124.6, 125.3, 126.0, 128.1, 128.38, 128.43, 130.0, 141.2, 150.9, 168.0; IR(neat) 1765, 1586, 1483, 1198, 1157, $756 \mathrm{~cm}^{-1}$; HRMS (EI) calcd for $\mathrm{C}_{18} \mathrm{H}_{18} \mathrm{O}_{2}$ $\left(\mathrm{M}^{+}\right)$266.1307, found 266.1304. Anal. Calcd for $\mathrm{C}_{18} \mathrm{H}_{18} \mathrm{O}_{2}: \mathrm{C}, 81.17 ; \mathrm{H}, 6.81$. Found: C, 81.18; H, 6.90 .

The enantiomeric excess was determined to be $94 \%$ by chiral HPLC [Daicel CHIRALCEL ${ }^{\circledR}$ OD-H column, hexane $: i-\mathrm{PrOH}=98: 2,0.6 \mathrm{~mL} / \mathrm{min}$, retention times: $t_{1}=35.9 \mathrm{~min}\left(\right.$ minor); $t_{2}=$ $42.9 \min$ (major)].<smiles>COc1ccc2c(c1)[C@](C)(CCc1ccccc1)CC(=O)O2</smiles>

6-Methoxy-4-methyl-4-phenethylchroman-2-one (2f). According to the general procedure, but using $3.5 \mathrm{~mol} \%[\mathrm{Rh}(\mathrm{OH})(\mathrm{cod})]_{2}$ and $8.0 \mathrm{~mol} \%(R)$-Tol-BINAP in toluene-THF $(4: 1), 2 \mathbf{2 f}$ $(54.8 \mathrm{mg}, 0.185 \mathrm{mmol}, 92 \%)$ was prepared from $1 f(59.3 \mathrm{mg}, 0.20 \mathrm{mmol}) .[\alpha]^{22}{ }_{\mathrm{D}}-10.3(c 2.3$, $\left.\mathrm{CHCl}_{3}\right) ;{ }^{1} \mathrm{H}$ NMR $(300 \mathrm{MHz}) \delta 1.43(\mathrm{~s}, 3 \mathrm{H}), 1.84-1.96(\mathrm{~m}, 2 \mathrm{H}), 2.43-2.55(\mathrm{~m}, 1 \mathrm{H}), 2.56-2.68(\mathrm{~m}$, $1 \mathrm{H}), 2.62(\mathrm{~d}, J=15.6 \mathrm{~Hz}, 1 \mathrm{H}), 2.78(\mathrm{~d}, J=15.6 \mathrm{~Hz}, 1 \mathrm{H}), 3.82(\mathrm{~s}, 3 \mathrm{H}), 6.78-6.86(\mathrm{~m}, 2 \mathrm{H}), 7.02$ $(\mathrm{d}, J=8.4 \mathrm{~Hz}, 1 \mathrm{H}), 7.08-7.29(\mathrm{~m}, 5 \mathrm{H}) ;{ }^{13} \mathrm{C}$ NMR $\delta 25.1,30.5,36.6,41.6,42.0,55.7,111.2$, 112.6, 117.9, 126.0, 128.1, 128.4, 131.1, 141.2, 144.8, 156.3, 168.2; HRMS (EI) calcd for $\mathrm{C}_{19} \mathrm{H}_{20} \mathrm{O}_{3}\left(\mathrm{M}^{+}\right)$296.1412, found 296.1415. Anal. Calcd for $\mathrm{C}_{19} \mathrm{H}_{20} \mathrm{O}_{3}: \mathrm{C}, 77.00 ; \mathrm{H}, 6.80$. Found: $\mathrm{C}$, 77.10; H, 6.57.

The enantiomeric excess was determined to be $95 \%$ by chiral HPLC [Daicel CHIRALCEL ${ }^{\circledR}$ OD-H column, hexane $: i-\mathrm{PrOH}=95: 5,0.6 \mathrm{~mL} / \mathrm{min}$, retention times: $t_{1}=41.2 \mathrm{~min}($ minor$) ; t_{2}=$ 48.5 min (major)]. 
<smiles>CCC1(C)CC(=O)Oc2ccccc21</smiles>

(R)-4-Ethyl-4-methylchroman-2-one (2g). According to the general procedure, $\mathbf{2 g}$ (30.4 $\mathrm{mg}$, $0.160 \mathrm{mmol}, 80 \%)$ was prepared from $1 \mathrm{~g}(38.0 \mathrm{mg}, 0.200 \mathrm{mmol}) .{ }^{1} \mathrm{H} \mathrm{NMR}(300 \mathrm{MHz}) \delta 0.84(\mathrm{t}$, $J=7.5 \mathrm{~Hz}, 3 \mathrm{H}), 1.33(\mathrm{~s}, 3 \mathrm{H}), 1.54-1.73(\mathrm{~m}, 2 \mathrm{H}), 2.56(\mathrm{~d}, J=15.8 \mathrm{~Hz}, 1 \mathrm{H}), 2.70(\mathrm{~d}, J=15.8 \mathrm{~Hz}$, $1 \mathrm{H})$, 7.02-7.08 $(\mathrm{m}, 1 \mathrm{H}), 7.10-7.18(\mathrm{~m}, 1 \mathrm{H}), 7.21-7.29(\mathrm{~m}, 2 \mathrm{H}) ;{ }^{13} \mathrm{C}$ NMR $\delta 8.4,24.7,32.7,36.5$, 41.3, 117.1, 124.4, 125.4, 128.1, 130.2, 150.9, 168.4; HRMS (EI) calcd for $\mathrm{C}_{12} \mathrm{H}_{14} \mathrm{O}_{2}\left(\mathrm{M}^{+}\right)$ 190.0994, found 190.0994. Anal. Calcd for $\mathrm{C}_{12} \mathrm{H}_{14} \mathrm{O}_{2}$ : C, 75.76; H, 7.42. Found: C, 76.00; H, 7.50 .

The product was transformed to ring-opened ester $\mathbf{D}$ to determine enantiomeric excess.

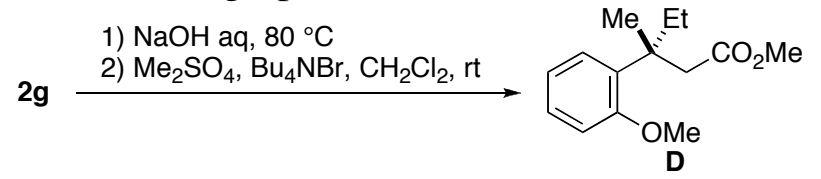

The enantiomeric excess was determined to be $94 \%$ by chiral HPLC [Daicel CHIRALCEL ${ }^{\circledR}$ OD-H column, hexane: $i$-PrOH $=98: 2,0.6 \mathrm{~mL} / \mathrm{min}$, retention times: $t_{1}=10.3 \mathrm{~min}(S) ; t_{2}=17.9$ $\min (R)]$.

The absolute configuration was assigned to be $R$ on the basis of the following relationship.
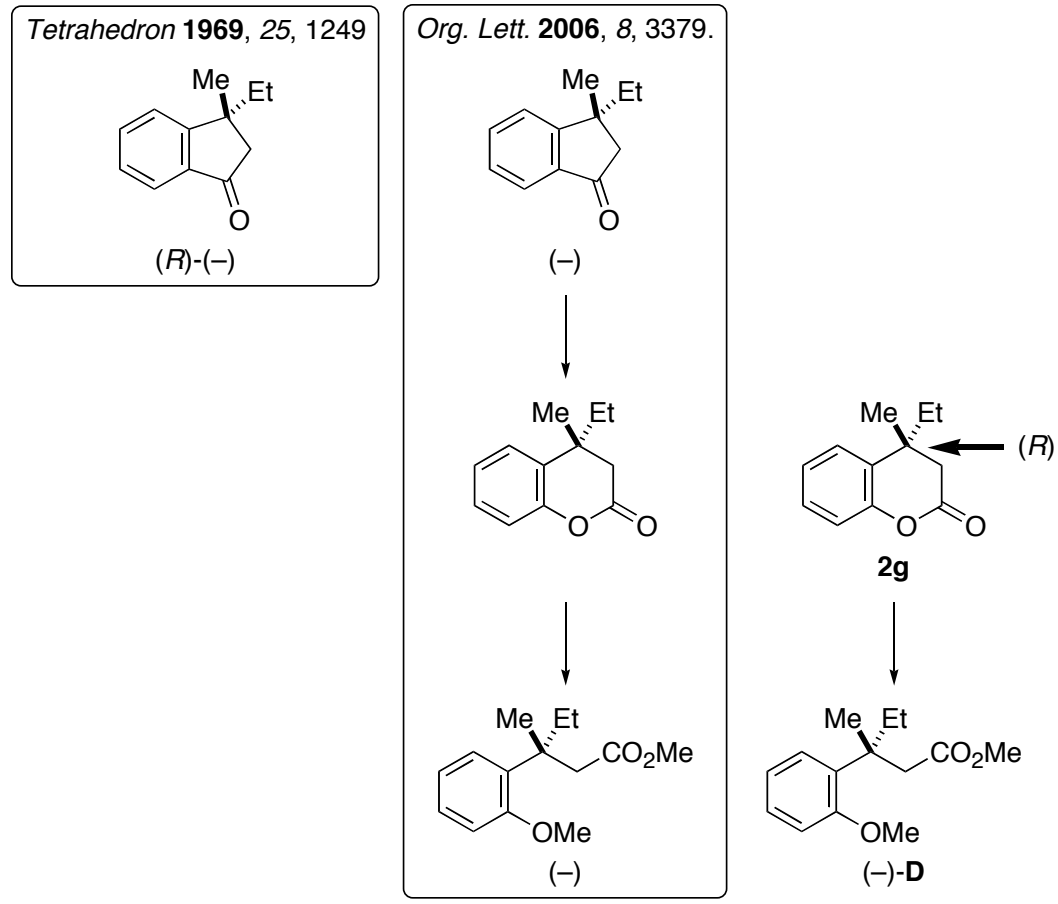<smiles>CCCC1(C)CC(=O)Oc2ccccc21</smiles>

4-Isopropyl-4-methylchroman-2-one (2h). According to the general procedure, $\mathbf{2 h} \mathbf{h} 35.6 \mathrm{mg}$, $0.174 \mathrm{mmol}, 87 \%)$ was prepared from $1 \mathrm{~h}(40.9 \mathrm{mg}, 0.200 \mathrm{mmol}) \cdot[\alpha]^{27}{ }_{\mathrm{D}}+18.1\left(c 1.8, \mathrm{CHCl}_{3}\right) ;{ }^{1} \mathrm{H}$ 
NMR $(300 \mathrm{MHz}) \delta 0.82(\mathrm{~d}, J=6.6 \mathrm{~Hz}, 3 \mathrm{H}), 0.92(\mathrm{~d}, J=6.9 \mathrm{~Hz}, 3 \mathrm{H}), 1.29(\mathrm{~s}, 3 \mathrm{H}), 1.85$ (sept, $J$ $=6.8 \mathrm{~Hz}, 1 \mathrm{H}), 2.49(\mathrm{~d}, J=15.9 \mathrm{~Hz}, 1 \mathrm{H}), 2.85(\mathrm{~d}, J=15.9 \mathrm{~Hz}, 1 \mathrm{H}), 7.01-7.07(\mathrm{~m}, 1 \mathrm{H}), 7.13(\mathrm{dt}, J$ $=1.0,7.5 \mathrm{~Hz}, 1 \mathrm{H}), 7.20-7.28(\mathrm{~m}, 2 \mathrm{H}) ;{ }^{13} \mathrm{C} \mathrm{NMR} \delta 17.1,17.5,20.9,34.6,39.4,39.6,117.0,124.1$, 126.3, 128.0, 130.5, 150.9, 168.5; HRMS (EI) calcd for $\mathrm{C}_{13} \mathrm{H}_{16} \mathrm{O}_{2}\left(\mathrm{M}^{+}\right)$204.1150, found 204.1153.

The enantiomeric excess was determined to be $93 \%$ by chiral HPLC [Daicel CHIRALCEL ${ }^{\circledR}$ OD-H column, hexane: $i-\mathrm{PrOH}=99: 1,0.6 \mathrm{~mL} / \mathrm{min}$, retention times: $t_{1}=15.0 \mathrm{~min}$ (minor); $t_{2}=$ 16.1 min (major)].

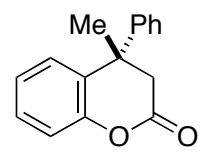

4-Methyl-4-phenylchroman-2-one (2i). According to the general procedure, but using 3.5 $\mathrm{mol} \%[\mathrm{Rh}(\mathrm{OH})(\mathrm{cod})]_{2}$ and $8.0 \mathrm{~mol} \%(R)$-Tol-BINAP in THF, $2 \mathbf{i}(32.3 \mathrm{mg}, 0.136 \mathrm{mmol}, 68 \%)$ was prepared from $1 \mathrm{i}(47.7 \mathrm{mg}, 0.200 \mathrm{mmol}) .[\alpha]^{28}{ }_{\mathrm{D}}-58.0\left(c 1.6, \mathrm{CHCl}_{3}\right) ;{ }^{1} \mathrm{H}$ NMR $(300 \mathrm{MHz}) \delta$ $1.75(\mathrm{~s}, 3 \mathrm{H}), 2.83(\mathrm{~d}, J=15.8 \mathrm{~Hz}, 1 \mathrm{H}), 3.29(\mathrm{~d}, J=15.8 \mathrm{~Hz}, 1 \mathrm{H}), 7.11(\mathrm{~d}, J=8.1 \mathrm{~Hz}, 1 \mathrm{H})$, 7.14-7.37 (m, 8H); ${ }^{13} \mathrm{C}$ NMR $\delta 27.6,41.1,43.8,99.9,117.3,124.7,126.1,126.6,127.1,128.7$, 130.8, 143.9, 151.1, 167.4; HRMS (EI) calcd for $\mathrm{C}_{16} \mathrm{H}_{14} \mathrm{O}_{2}\left(\mathrm{M}^{+}\right)$238.0994, found 238.0991. Anal. Calcd for $\mathrm{C}_{16} \mathrm{H}_{14} \mathrm{O}_{2}$ : C, 80.65; H, 5.92. Found: C, 80.45; H, 5.81.

The enantiomeric excess was determined to be $92 \%$ by chiral HPLC [Daicel CHIRALCEL ${ }^{\circledR}$ OD-H, hexane: $i$ PrOH $=98: 2,0.6 \mathrm{~mL} / \mathrm{min}$, retention times: $t_{1}=27.9$ min (major); $t_{2}=30.5 \mathrm{~min}$ (minor)].

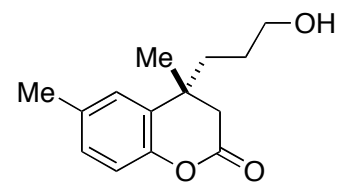

(R)-4-(3-Hydroxypropyl)-4,6-dimethylchroman-2-one (2j). According to the general procedure, but in toluene-THF (4:1), $\mathbf{2} \mathbf{j}(36.2 \mathrm{mg}, 0.155 \mathrm{mmol}, 77 \%)$ was prepared from $\mathbf{1 i}(46.9$ $\mathrm{mg}, 0.200 \mathrm{mmol}) .[\alpha]_{\mathrm{D}}^{23}+1.1\left(c 1.4, \mathrm{CHCl}_{3}\right) ;{ }^{1} \mathrm{H}$ NMR $(300 \mathrm{MHz}) \delta 1.36(\mathrm{~s}, 3 \mathrm{H}), 1.38-1.70(\mathrm{~m}$, $4 \mathrm{H}), 2.34(\mathrm{~s}, 3 \mathrm{H}), 2.57(\mathrm{~d}, J=15.6 \mathrm{~Hz}, 1 \mathrm{H}), 2.69(\mathrm{~d}, J=15.6 \mathrm{~Hz}, 1 \mathrm{H}), 3.58(\mathrm{t}, J=6.3 \mathrm{~Hz}, 2 \mathrm{H})$, 6.92-6.96 (m, 1H), 7.01-7.08 (m, 2H); ${ }^{13} \mathrm{C}$ NMR $\delta$ 21.0, 25.1, 27.3, 36.0, 36.4, 41.9, 62.8, 116.9, 125.7, 128.8, 129.7, 134.1, 148.7, 168.5; HRMS (EI) calcd for $\mathrm{C}_{14} \mathrm{H}_{18} \mathrm{O}_{3}\left(\mathrm{M}^{+}\right)$234.1256, found 234.1266.

The enantiomeric excess was determined to be $77 \%$ by chiral HPLC [Daicel CHIRALCEL ${ }^{\circledR}$ OD-H, hexane: $i-\mathrm{PrOH}=95: 5,0.6 \mathrm{~mL} / \mathrm{min}$, retention times: $t_{1}=45.6 \mathrm{~min}$ (major); $t_{2}=49.6 \mathrm{~min}$ (minor)]. 
Rhodium-Catalyzed Cascade Reaction of 1 with Electron-Deficient Alkenes 12 (Table 2)

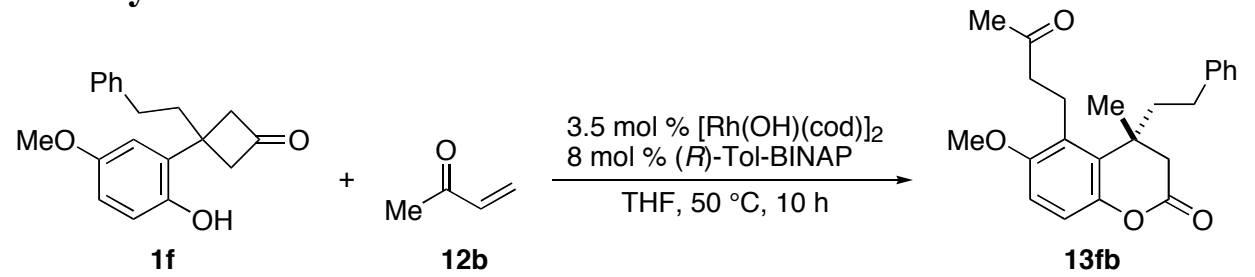

6-Methoxy-4-methyl-5-(3-oxobutyl)-4-phenethylchroman-2-one (13fb). To a mixture of $[\mathrm{RhOH}(\mathrm{cod})]_{2}(3.20 \mathrm{mg}, 7.0 \mu \mathrm{mol}, 3.5 \mathrm{~mol} \%)$ and $(R)$-Tol-BINAP (10.9 $\mathrm{mg}, 16.1 \mu \mathrm{mol}, 8.1$ mol \%) in THF (1.4 mL) was added 3-buten-2-one (12b, $140 \mathrm{mg}, 2.00 \mathrm{mmol})$. Cyclobutanone $\mathbf{1 f}$ $(59.3 \mathrm{mg}, 0.200 \mathrm{mmol})$ in THF $(0.6 \mathrm{~mL})$ was then added dropwise to the reaction mixture at $50{ }^{\circ} \mathrm{C}$ over $1 \mathrm{~h}$ using syringe pump. After being stirred for $10 \mathrm{~h}$ at $50{ }^{\circ} \mathrm{C}$, the reaction mixture was filtered through a pad of Florisil ${ }^{\circledR}\left(\mathrm{Et}_{2} \mathrm{O}-\mathrm{AcOEt}\right)$. The filtrate was concentrated, and the residue was purified by preparative thin-layer chromatography of silica gel (hexane:AcOEt $=3: 1$ ) to afford 13fb (47.4 mg, $0.129 \mathrm{mmol}, 65 \%):[\alpha]^{19}{ }_{\mathrm{D}}-25.4\left(c\right.$ 2.2, $\left.\mathrm{CHCl}_{3}\right) ;{ }^{1} \mathrm{H} \mathrm{NMR}(300 \mathrm{MHz}) \delta$ $1.58(\mathrm{~s}, 3 \mathrm{H}), 1.86-2.08(\mathrm{~m}, 2 \mathrm{H}), 2.16(\mathrm{~s}, 3 \mathrm{H}), 2.47-2.67(\mathrm{~m}, 3 \mathrm{H}), 2.62(\mathrm{~d}, J=15.8 \mathrm{~Hz}, 1 \mathrm{H}), 2.75$ $(\mathrm{d}, J=15.8 \mathrm{~Hz}, 1 \mathrm{H}), 2.85-3.10(\mathrm{~m}, 3 \mathrm{H}), 3.81(\mathrm{~s}, 3 \mathrm{H}), 6.78(\mathrm{~d}, J=9.0 \mathrm{~Hz}, 1 \mathrm{H}), 6.95(\mathrm{~d}, J=8.7$ $\mathrm{Hz}, 1 \mathrm{H}), 7.07-7.19(\mathrm{~m}, 3 \mathrm{H}), 7.20-7.25(\mathrm{~m}, 2 \mathrm{H}) ;{ }^{13} \mathrm{C}$ NMR $\delta 22.4,27.5,29.8,30.6,39.1,42.6$, 43.3, 43.4, 55.7, 109.6, 116.0, 126.0, 128.1, 128.3, 128.4, 129.9, 141.0, 145.7, 155.1, 168.3, 208.0; HRMS (EI) calcd for $\mathrm{C}_{23} \mathrm{H}_{26} \mathrm{O}_{4}\left(\mathrm{M}^{+}\right) 366.1831$, found 366.1823.

The enantiomeric excess was determined to be $96 \%$ by chiral HPLC [Daicel CHIRALCEL ${ }^{\circledR}$ OD-H column, hexane: $i-\mathrm{PrOH}=80: 20,0.6 \mathrm{~mL} / \mathrm{min}$, retention times: $t_{1}=21.3 \mathrm{~min}\left(\right.$ minor); $t_{2}=$ $28.6 \min$ (major)].<smiles>COc1ccc2c(c1CCC#N)C(C)(CCc1ccccc1)CC(=O)O2</smiles>

5-(Cyanoethyl)-6-methoxy-4-methyl-4-phenethylchroman-2-one (13fa). According to the procedure analogous to that described for $\mathbf{1 3 f b}, \mathbf{1 3 f a}(65.0 \mathrm{mg}, 0.186 \mathrm{mmol}, 93 \%)$ was prepared from 1 f $(59.3 \mathrm{mg}, 0.200 \mathrm{mmol})$ and acrylonitrile (12a, $108 \mathrm{mg}, 2.04 \mathrm{mmol}) .[\alpha]_{\mathrm{D}}^{22}-32.4(c) 3.0$, $\left.\mathrm{CHCl}_{3}\right) ;{ }^{1} \mathrm{H}$ NMR $(300 \mathrm{MHz}) \delta 1.66(\mathrm{~s}, 3 \mathrm{H}), 1.87-2.06(\mathrm{~m}, 2 \mathrm{H}), 2.47-2.62(\mathrm{~m}, 3 \mathrm{H}), 2.66(\mathrm{~d}, J=$ $15.9 \mathrm{~Hz}, 1 \mathrm{H}), 2.77(\mathrm{~d}, J=15.9 \mathrm{~Hz}, 1 \mathrm{H}), 2.81(\mathrm{ddd}, J=16.6,10.3,6.1 \mathrm{~Hz}, 1 \mathrm{H}), 3.10-3.30(\mathrm{~m}$, $2 \mathrm{H}), 3.85(\mathrm{~s}, 3 \mathrm{H}), 6.81(\mathrm{~d}, J=8.9 \mathrm{~Hz}, 1 \mathrm{H}), 7.01(\mathrm{~d}, J=8.9 \mathrm{~Hz}, 1 \mathrm{H}), 7.06-7.13(\mathrm{~m}, 2 \mathrm{H})$, 7.13-7.20 (m, 1H), 7.21-7.29 (m, 2H); ${ }^{13} \mathrm{C}$ NMR $\delta 16.7,24.7,27.7,30.6,39.1,42.7,43.1,55.8$, $109.9,117.3,119.4,125.1,126.0,128.1,128.4,130.3,140.6,145.6,154.9,167.8$; HRMS (CI) calcd for $\mathrm{C}_{22} \mathrm{H}_{23} \mathrm{NO}_{3}\left(\mathrm{M}^{+}\right) 349.1678$, found 349.1677.

The enantiomeric excess was determined to be $95 \%$ by chiral HPLC [Daicel CHIRALCEL ${ }^{\circledR}$ OD-H column, hexane: $i-\mathrm{PrOH}=80: 20,0.6 \mathrm{~mL} / \mathrm{min}$, retention times: $t_{1}=32.1 \mathrm{~min}(\mathrm{minor}) ; t_{2}=$ 38.1 min (major)]. 


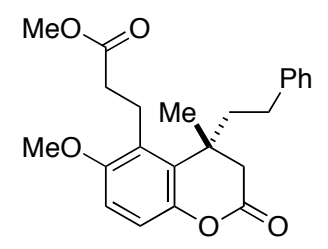

Methyl 3-(6-methoxy-4-methyl-2-oxo-4-phenethylchroman-5-yl)propanoate (13fc). According to the procedure analogous to that described for 13fb, 13fc $(58.0 \mathrm{mg}, 0.152 \mathrm{mmol}$, $76 \%$ ) was prepared from 1 f $(59.3 \mathrm{mg}, 0.20 \mathrm{mmol})$ and methyl acrylate (12c, $172 \mathrm{mg}, 2.00$ mmol). $[\alpha]^{23}-32.6\left(c 2.5, \mathrm{CHCl}_{3}\right) ;{ }^{1} \mathrm{H}$ NMR $(300 \mathrm{MHz}) \delta 1.64(\mathrm{~s}, 3 \mathrm{H}), 1.88-2.10(\mathrm{~m}, 2 \mathrm{H})$, 2.40-2.64 (m, 3H), $2.64(\mathrm{~d}, J=15.9 \mathrm{~Hz}, 1 \mathrm{H}), 2.75(\mathrm{~d}, J=15.9 \mathrm{~Hz}, 1 \mathrm{H}), 2.75-2.88(\mathrm{~m}, 1 \mathrm{H})$, 3.02-3.22 (m, 2H), $3.72(\mathrm{~s}, 3 \mathrm{H}), 3.82(\mathrm{~s}, 3 \mathrm{H}), 6.78(\mathrm{~d}, J=8.7 \mathrm{~Hz}, 1 \mathrm{H}), 6.96(\mathrm{~d}, J=8.7 \mathrm{~Hz}, 1 \mathrm{H})$, 7.07-7.27 (m, 5H); ${ }^{13} \mathrm{C}$ NMR $\delta 23.8,27.6,30.7,33.6,39.1,42.6,43.3,51.7,55.7,109.6,116.2$, $125.9,127.6,128.1,128.3,130.0,141.0,145.6,155.1,168.2,173.5$; HRMS (CI) calcd for $\mathrm{C}_{23} \mathrm{H}_{26} \mathrm{O}_{5}\left(\mathrm{M}^{+}\right)$382.1780, found 382.1776.

The enantiomeric excess was determined to be $95 \%$ by chiral HPLC [Daicel CHIRALCEL ${ }^{\circledR}$ OD-H column, hexane: $i-\mathrm{PrOH}=85: 15,0.6 \mathrm{~mL} / \mathrm{min}$, retention times: $t_{1}=19.7 \mathrm{~min}(\mathrm{minor}) ; t_{2}=$ 23.8 min (major)].<smiles>CCC1(C)CC(=O)Oc2cccc(CCC(C)=O)c21</smiles>

4-Ethyl-4-methyl-5-(3-oxobutyl)chroman-2-one (13gb). According to the procedure analogous to that described for 13fb, 13gb $(39.1 \mathrm{mg}, 0.150 \mathrm{mmol}, 75 \%)$ was prepared from $\mathbf{1 g}$ (38.0 mg, $0.200 \mathrm{mmol})$ and $12 \mathrm{~b}(142 \mathrm{mg}, 2.03 \mathrm{mmol}) .[\alpha]_{\mathrm{D}}^{22}-0.85\left(c 0.71, \mathrm{CHCl}_{3}\right) ;{ }^{1} \mathrm{H} \mathrm{NMR}$ $(300 \mathrm{MHz}) \delta 0.89(\mathrm{t}, J=7.2 \mathrm{~Hz}, 3 \mathrm{H}), 1.49(\mathrm{~s}, 3 \mathrm{H}), 1.68(\mathrm{sext}, J=7.4 \mathrm{~Hz}, 1 \mathrm{H}), 1.84(\mathrm{sext}, J=7.4$ $\mathrm{Hz}, 1 \mathrm{H}), 2.20(\mathrm{~s}, 3 \mathrm{H}), 2.54(\mathrm{~d}, J=15.9 \mathrm{~Hz}, 1 \mathrm{H}), 2.68(\mathrm{~d}, J=15.9 \mathrm{~Hz}, 1 \mathrm{H}), 2.82(\mathrm{t}, J=8.0 \mathrm{~Hz}$, $2 \mathrm{H}), 2.94-3.18(\mathrm{~m}, 2 \mathrm{H}), 6.92(\mathrm{~d}, J=8.1 \mathrm{~Hz}, 1 \mathrm{H}+1 \mathrm{H}), 7.11(\mathrm{~s}, 1 \mathrm{H}), 7.17(\mathrm{t}, J=7.8 \mathrm{~Hz}, 1 \mathrm{H}) ;{ }^{13} \mathrm{C}$ NMR $\delta 8.8,26.9,27.8,30.1,32.9,38.9,42.5,45.3,116.0,126.7,127.8,128.8,140.0,152.1$, 168.3, 207.1; HRMS (EI) calcd for $\mathrm{C}_{16} \mathrm{H}_{20} \mathrm{O}_{3}\left(\mathrm{M}^{+}\right)$260.1412, found 260.1412.

The enantiomeric excess was determined to be $97 \%$ by chiral HPLC [Daicel CHIRALCEL ${ }^{\circledR}$ AS-H column, hexane: $i-\mathrm{PrOH}=80: 20,0.6 \mathrm{~mL} / \mathrm{min}$, retention times: $t_{1}=27.9 \mathrm{~min}$ (major); $t_{2}=$ 38.6 min (minor)].

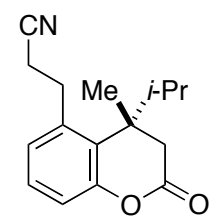

5-(Cyanoethyl)-4-isopropyl-4-methylchroman-2-one (13ha). According to the procedure analogous to that described for $\mathbf{1 3 f b}$, 13ha $(45.7 \mathrm{mg}, 0.178 \mathrm{mmol}, 89 \%)$ was prepared from $\mathbf{1 h}$ (40.9 mg, $0.202 \mathrm{mmol})$ and 12a $(106 \mathrm{mg}, 2.0 \mathrm{mmol}) .[\alpha]_{\mathrm{D}}^{20}-0.70\left(c\right.$ 2.3, $\left.\mathrm{CHCl}_{3}\right) ;{ }^{1} \mathrm{H} \mathrm{NMR}(300$ MHz) $\delta 0.88(\mathrm{~d}, J=6.9 \mathrm{~Hz}, 3 \mathrm{H}), 0.97(\mathrm{~d}, J=6.9 \mathrm{~Hz}, 3 \mathrm{H}), 1.49(\mathrm{~s}, 3 \mathrm{H}), 2.01$ (sept, $J=6.9 \mathrm{~Hz}$, 
$1 \mathrm{H}), 2.49(\mathrm{~d}, J=15.9 \mathrm{~Hz}, 1 \mathrm{H}), 2.69(\mathrm{dd}, J=8.6,6.2 \mathrm{~Hz}, 1 \mathrm{H}), 2.71(\mathrm{dd}, J=8.6,4.4 \mathrm{~Hz}, 1 \mathrm{H}), 2.78$ $(\mathrm{d}, J=15.9 \mathrm{~Hz}, 1 \mathrm{H}), 3.02-3.15(\mathrm{~m}, 1 \mathrm{H}), 3.24-3.36(\mathrm{~m}, 1 \mathrm{H}), 6.96-7.04(\mathrm{~m}, 2 \mathrm{H}), 7.25(\mathrm{t}, J=7.8$ $\mathrm{Hz}, 1 \mathrm{H}) ;{ }^{13} \mathrm{C}$ NMR $\delta 17.3,17.5,19.2,25.0,30.0,35.6,39.9,41.9,117.2,118.8,126.8,128.3$, 129.9, 137.2, 152.2, 168.0; HRMS (CI) calcd for $\mathrm{C}_{16} \mathrm{H}_{19} \mathrm{NO}_{2}\left(\mathrm{M}^{+}\right)$257.1416, found 257.1419.

The enantiomeric excess was determined to be $91 \%$ by chiral HPLC [Shimadzu IB column, hexane: $\mathrm{ClCH}_{2} \mathrm{CH}_{2} \mathrm{Cl}: i-\mathrm{PrOH}=82.5: 16.5: 1,0.6 \mathrm{~mL} / \mathrm{min}$, retention times: $t_{1}=19.3 \mathrm{~min}$ (major); $t_{2}$ $=21.5 \mathrm{~min}$ (minor)].

\section{Deuterium Labeling Experiment with 1a (Scheme 2)}

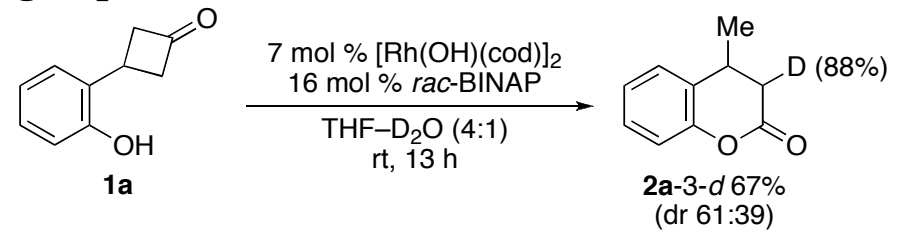

A mixture of $[\mathrm{Rh}(\mathrm{OH})(\mathrm{cod})]_{2}(6.38 \mathrm{mg}, 14.0 \mu \mathrm{mol}, 7.0 \mathrm{~mol} \%)$, rac-BINAP $(20.0 \mathrm{mg}, 32.1$ $\mu \mathrm{mol}, 16.1 \mathrm{~mol} \%)$, and $1 \mathrm{a}(32.4 \mathrm{mg}, 0.200 \mathrm{mmol})$ in $\mathrm{THF}-\mathrm{D}_{2} \mathrm{O}(4: 1,2.0 \mathrm{~mL})$ was stirred at room temperature for $13 \mathrm{~h}$. The reaction mixture was filtered through a pad of Florisil ${ }^{\circledR}$ $\left(\mathrm{Et}_{2} \mathrm{O}-\mathrm{AcOEt}\right)$ and concentrated. The residue was purified by preparative thin-layer chromatography of silica gel (hexane:AcOEt $=8: 1)$ to afford 2a-3-d $(21.8 \mathrm{mg}, 0.134 \mathrm{mmol}$, $67 \%$; $88 \%$ D, dr 61:39 by ${ }^{1} \mathrm{H}$ NMR). ${ }^{1} \mathrm{H}$ NMR $(300 \mathrm{MHz}) \delta 1.34(\mathrm{~d}, J=7.2 \mathrm{~Hz}$, minor $3 \mathrm{H}), 1.34$ (d, $J=7.2 \mathrm{~Hz}$, major $3 \mathrm{H}), 2.56(\mathrm{dt}, J=7.4,2.1 \mathrm{~Hz}$, major $1 \mathrm{H}), 2.83(\mathrm{dt}, J=5.0,2.3 \mathrm{~Hz}$, minor $1 \mathrm{H}$ ), 3.18 (quint, $J=6.9 \mathrm{~Hz}, 1 \mathrm{H}), 7.06(\mathrm{dd}, J=7.7,0.9 \mathrm{~Hz}, 1 \mathrm{H}), 7.13(\mathrm{dt}, J=1.2,7.5 \mathrm{~Hz}, 1 \mathrm{H})$, 7.21-7.30 (m, 2H); ${ }^{13} \mathrm{C}$ NMR $\delta 19.8$ (major), 19.9 (minor), 29.3 (major), 29.4 (minor), $36.4\left({ }^{1} J_{\mathrm{C}-\mathrm{D}}\right.$ $=20.3 \mathrm{~Hz}$, minor), $36.5\left({ }^{1} J_{\mathrm{C}-\mathrm{D}}=20.3 \mathrm{~Hz}\right.$, major), 116.9, 124.5, 126.4 (major), 126.5 (minor), 127.8, 128.2, 151.1, 168.3; HRMS (EI) calcd for $\mathrm{C}_{10} \mathrm{H}_{9} \mathrm{DO}_{2}\left(\mathrm{M}^{+}\right)$163.0743, found 163.0744.

\section{Deuterium Labeling Experiment with 1f (Scheme 3)}

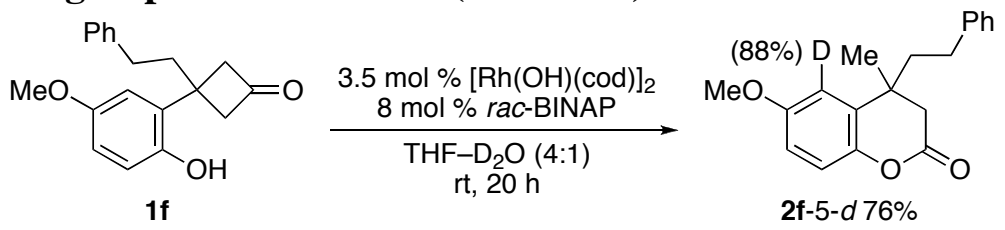

A mixture of $[\mathrm{Rh}(\mathrm{OH})(\mathrm{cod})]_{2}(6.36 \mathrm{mg}, 13.9 \mu \mathrm{mol}, 3.5 \mathrm{~mol} \%), \mathrm{rac}$-BINAP $(19.9 \mathrm{mg}, 32.0$ $\mu \mathrm{mol}, 8.0 \mathrm{~mol} \%)$, and $\mathbf{1 f}(119 \mathrm{mg}, 0.402 \mathrm{mmol})$ in THF- $\mathrm{D}_{2} \mathrm{O}(4: 1,4.0 \mathrm{~mL})$ was stirred at room temperature for $20 \mathrm{~h}$. The reaction mixture was filtered through a pad of Florisil ${ }^{\circledR}\left(\mathrm{Et}_{2} \mathrm{O}-\mathrm{AcOEt}\right)$ and concentrated. The residue was purified by preparative thin-layer chromatography of silica gel (hexane:AcOEt $=6: 1)$ to afford $\mathbf{2 f}-5-d\left(90.5 \mathrm{mg}, 0.304 \mathrm{mmol}, 76 \% ; 88 \%\right.$ D by $\left.{ }^{1} \mathrm{H} \mathrm{NMR}\right) .{ }^{1} \mathrm{H}$ NMR (300 MHz) $\delta 1.42(\mathrm{~s}, 3 \mathrm{H}), 1.82-1.94(\mathrm{~m}, 2 \mathrm{H}), 2.41-2.54(\mathrm{~m}, 1 \mathrm{H}), 2.55-2.67(\mathrm{~m}, 1 \mathrm{H}), 2.62$ $(\mathrm{d}, J=15.5 \mathrm{~Hz}, 1 \mathrm{H}), 2.78(\mathrm{~d}, J=15.5 \mathrm{~Hz}, 1 \mathrm{H}), 3.82(\mathrm{~s}, 3 \mathrm{H}), 6.80(\mathrm{~d}, J=8.7 \mathrm{~Hz}, 1 \mathrm{H}), 6.84(\mathrm{~d}, J$ $=2.4 \mathrm{~Hz}, 0.12 \mathrm{H}), 7.02(\mathrm{~d}, J=8.7 \mathrm{~Hz}, 1 \mathrm{H}), 7.07-7.13(\mathrm{~m}, 2 \mathrm{H}), 7.13-7.20(\mathrm{~m}, 1 \mathrm{H}), 7.22-7.29(\mathrm{~m}$, $2 \mathrm{H}) ;{ }^{13} \mathrm{C}$ NMR $\delta 25.1,30.5,36.6,41.6,42.0,55.7,110.9\left({ }^{1} J_{\mathrm{C}-\mathrm{D}}=23.8 \mathrm{~Hz}\right), 112.5,117.8,126.0$, 128.1, 128.4, 131.0, 141.2, 144.7, 156.2, 168.2; HRMS (EI) calcd for $\mathrm{C}_{19} \mathrm{H}_{19} \mathrm{DO}_{3}\left(\mathrm{M}^{+}\right)$297.1474, 
found 297.1483. 


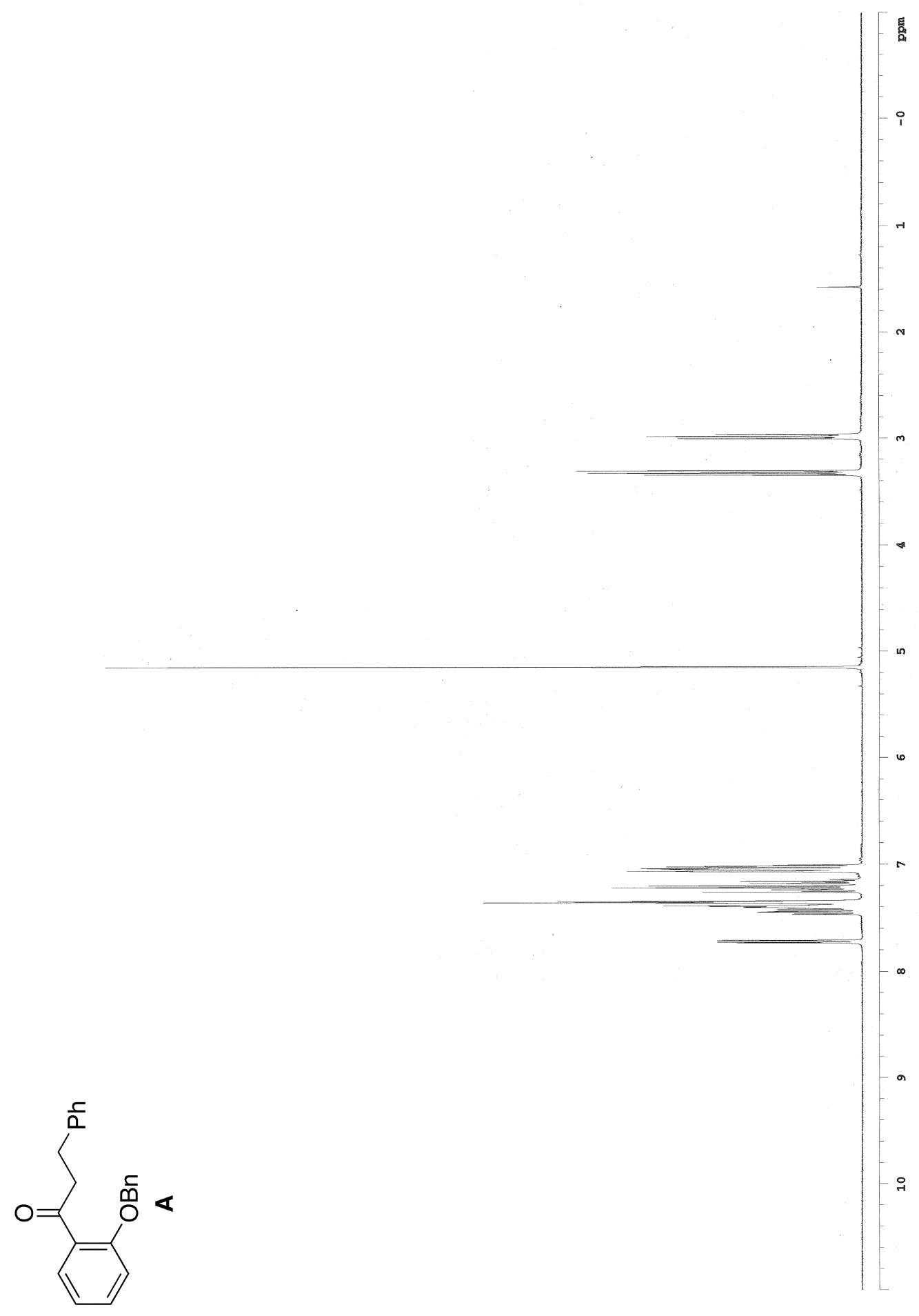




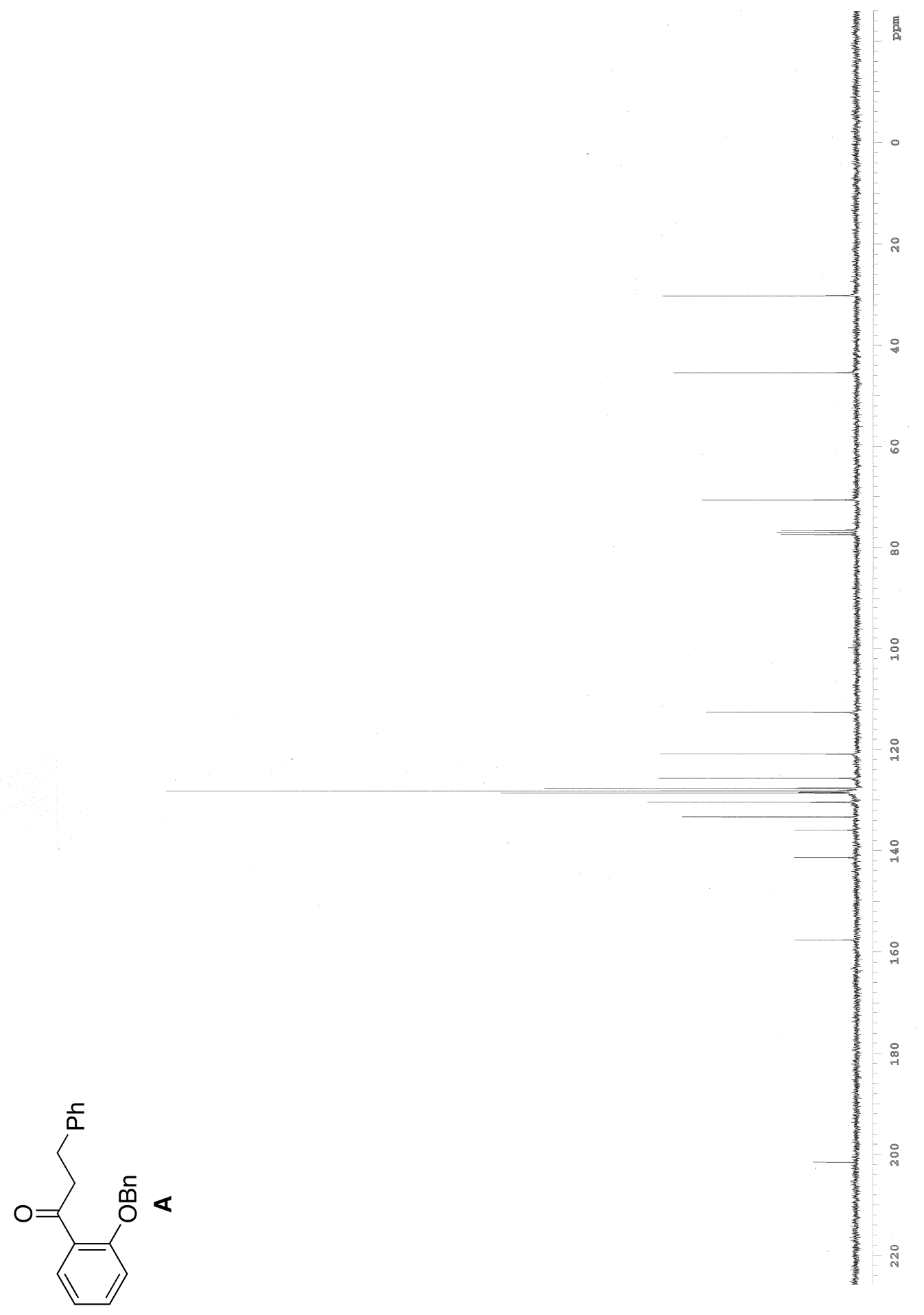




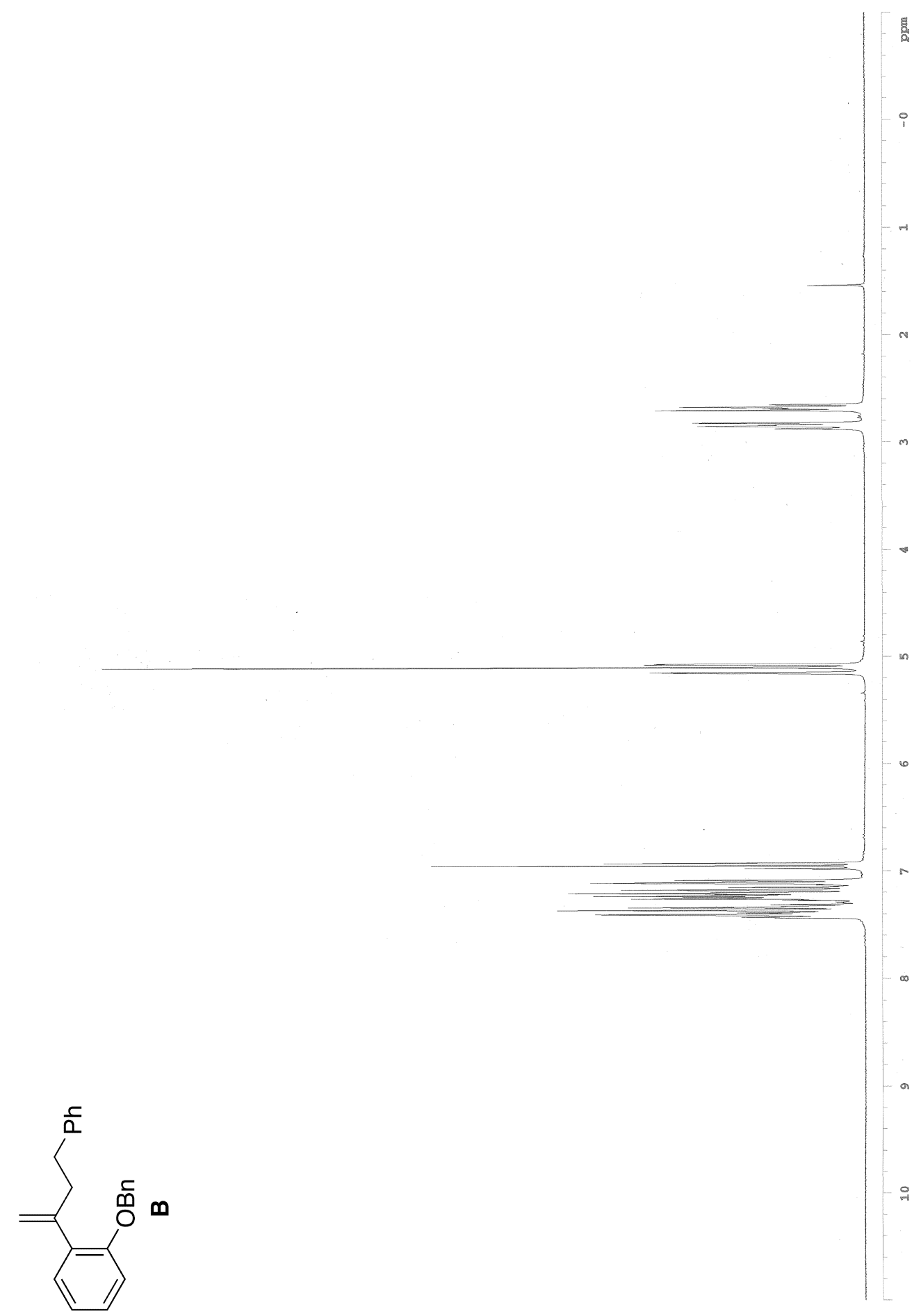




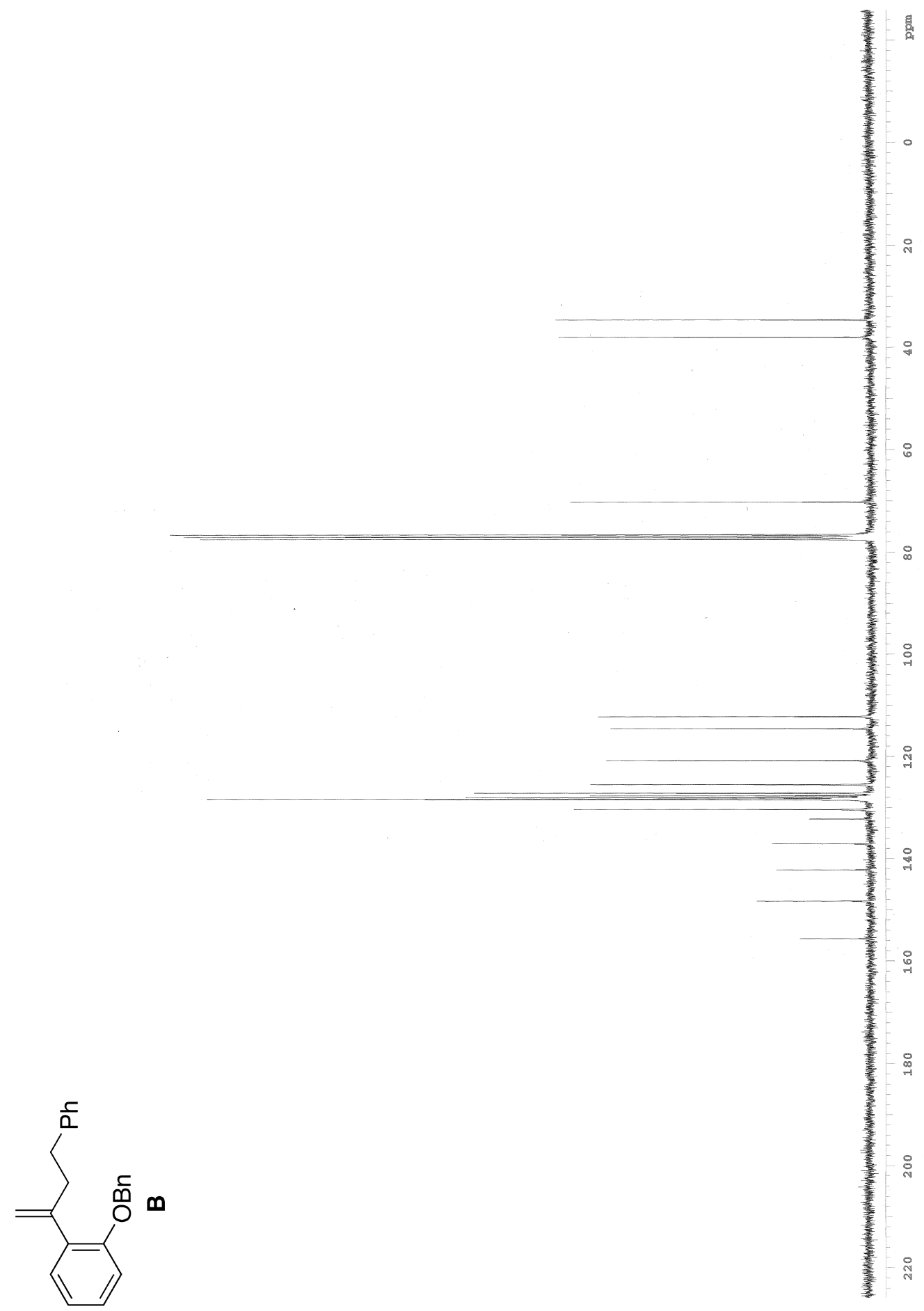




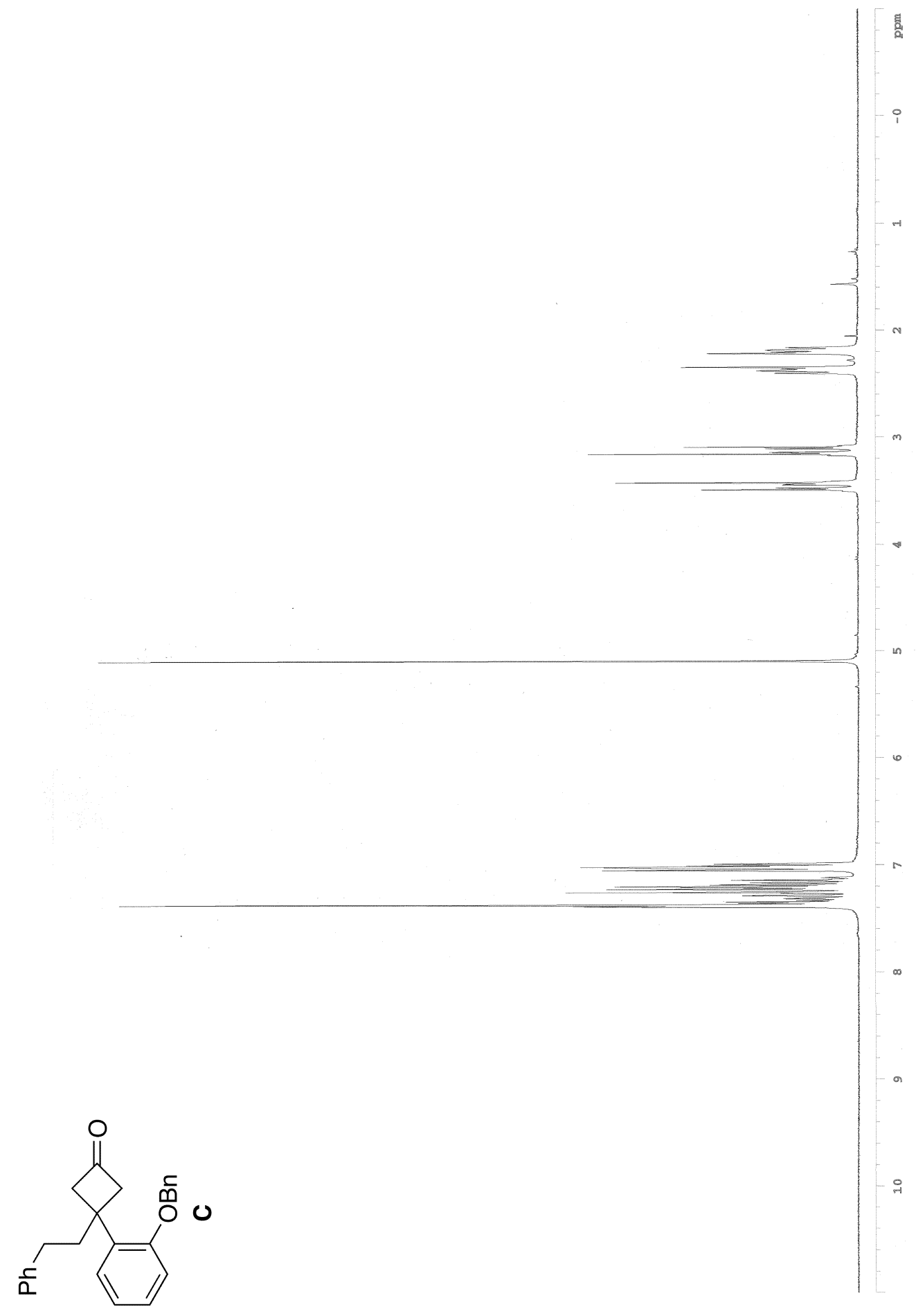




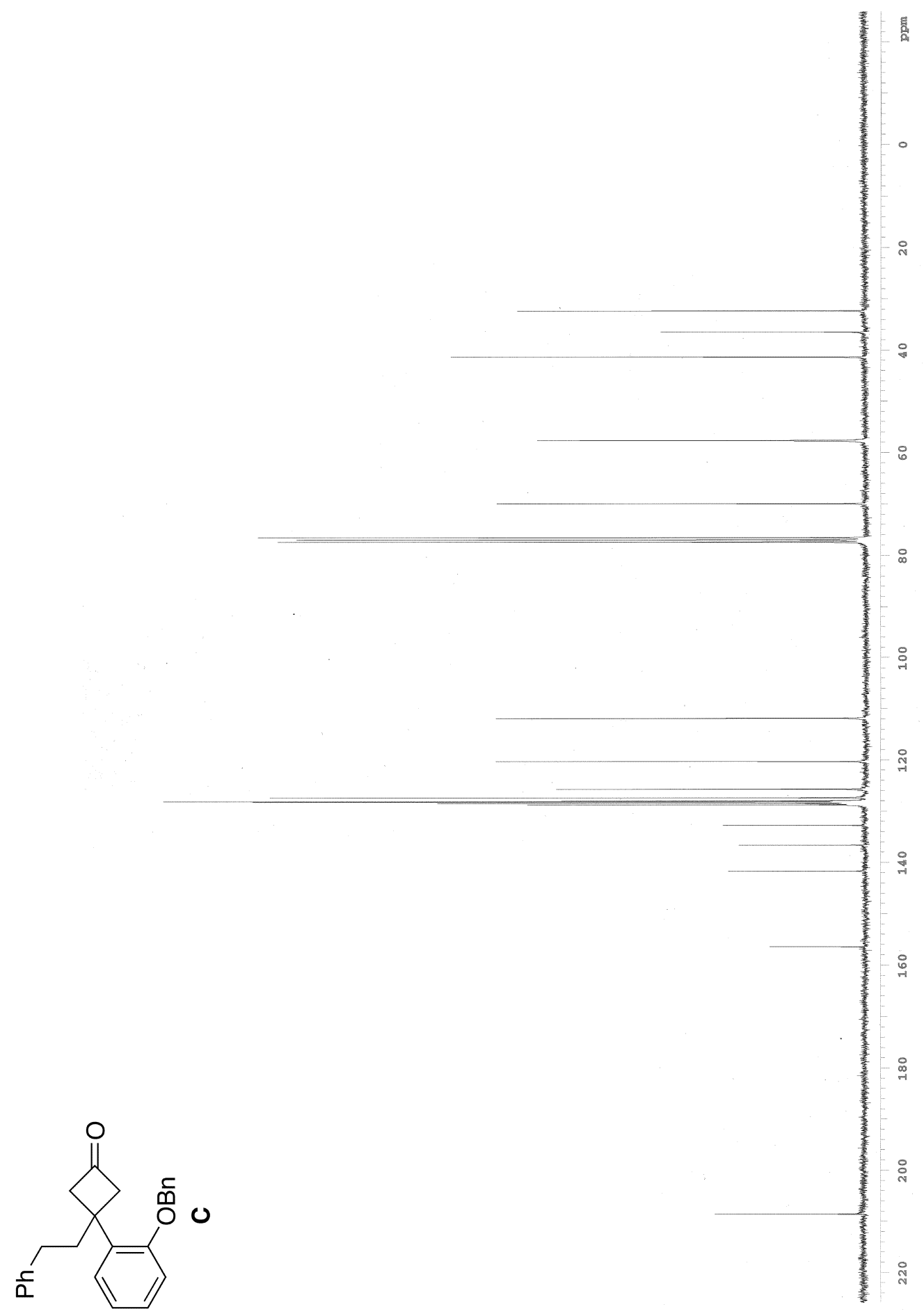




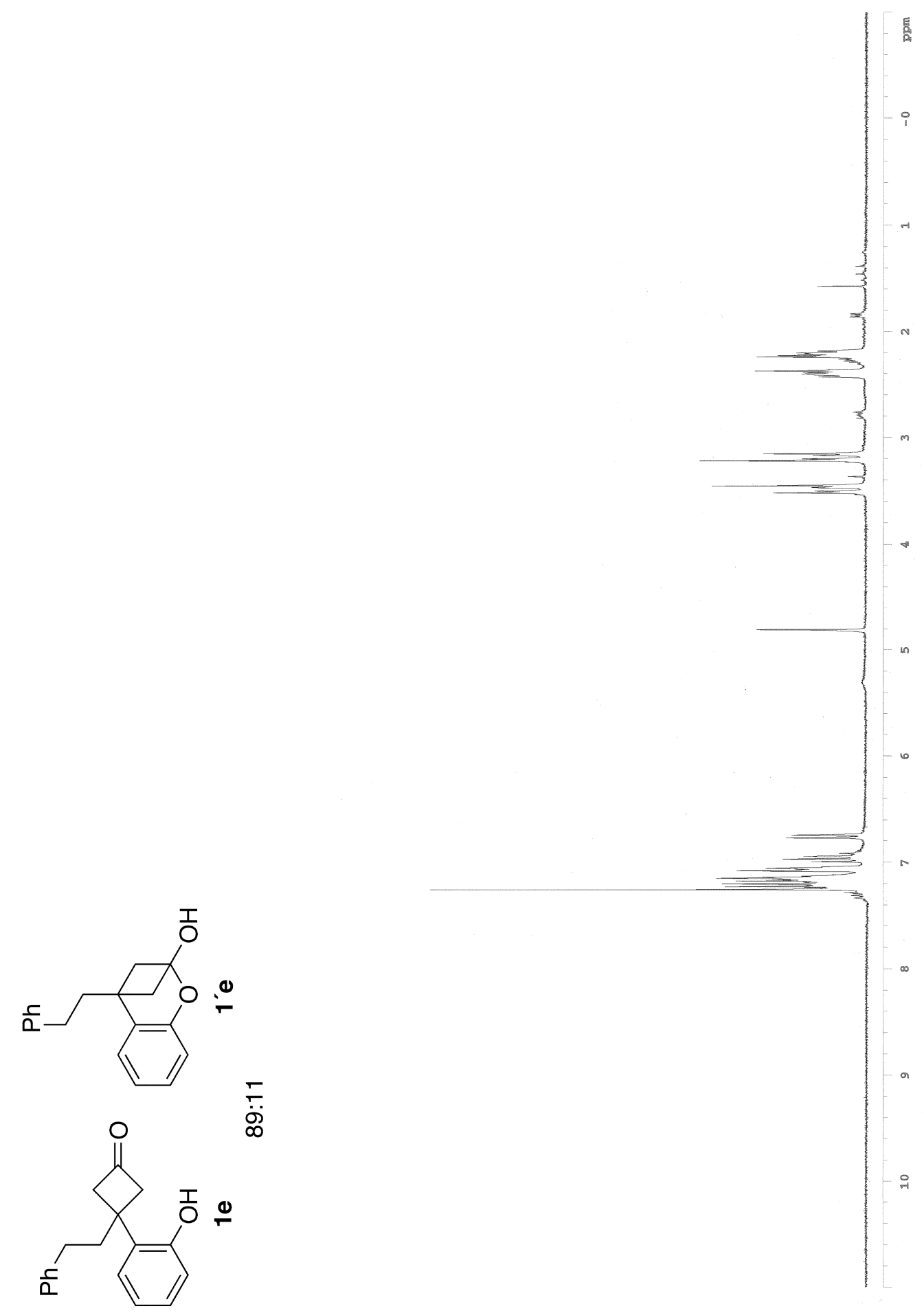




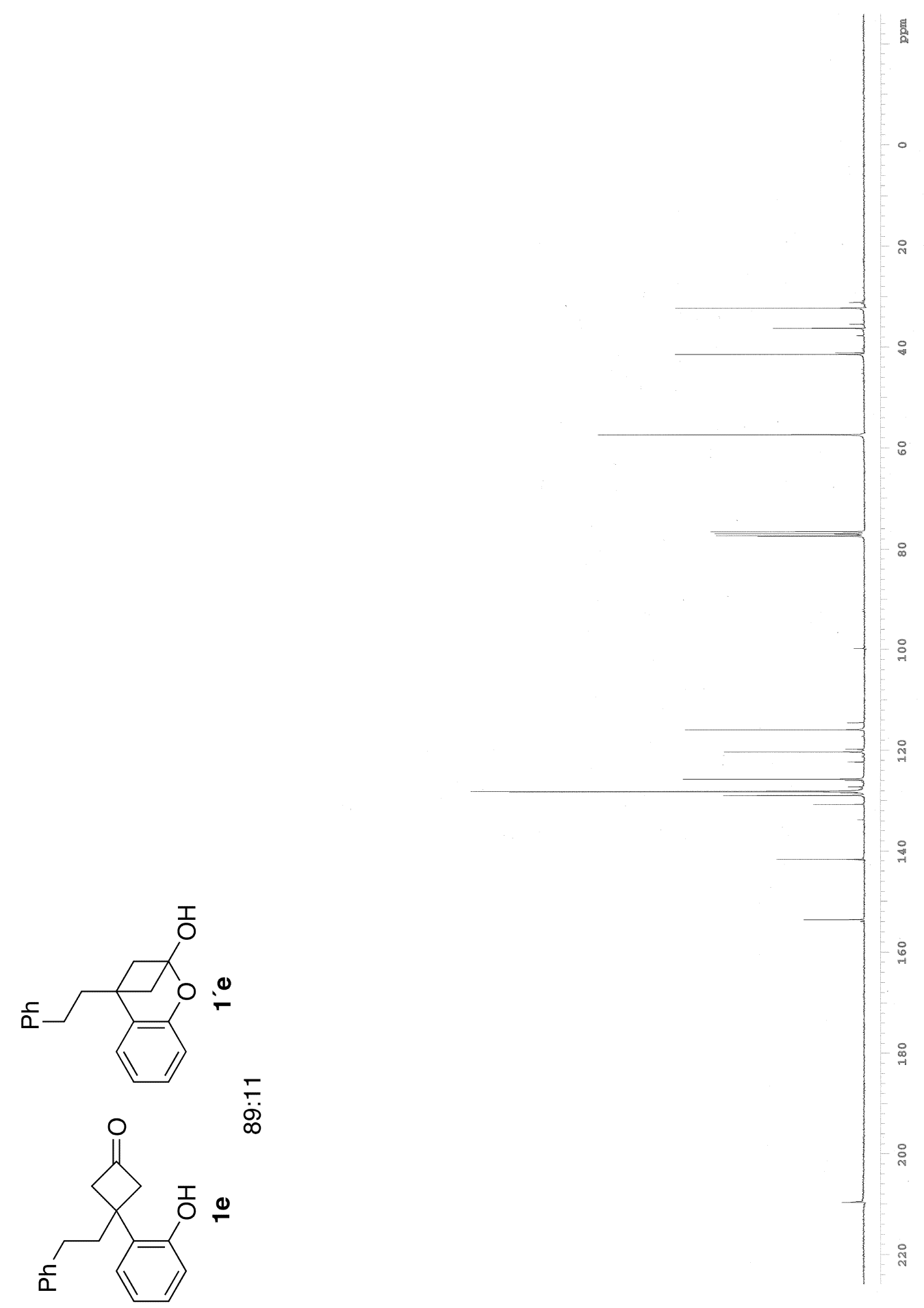




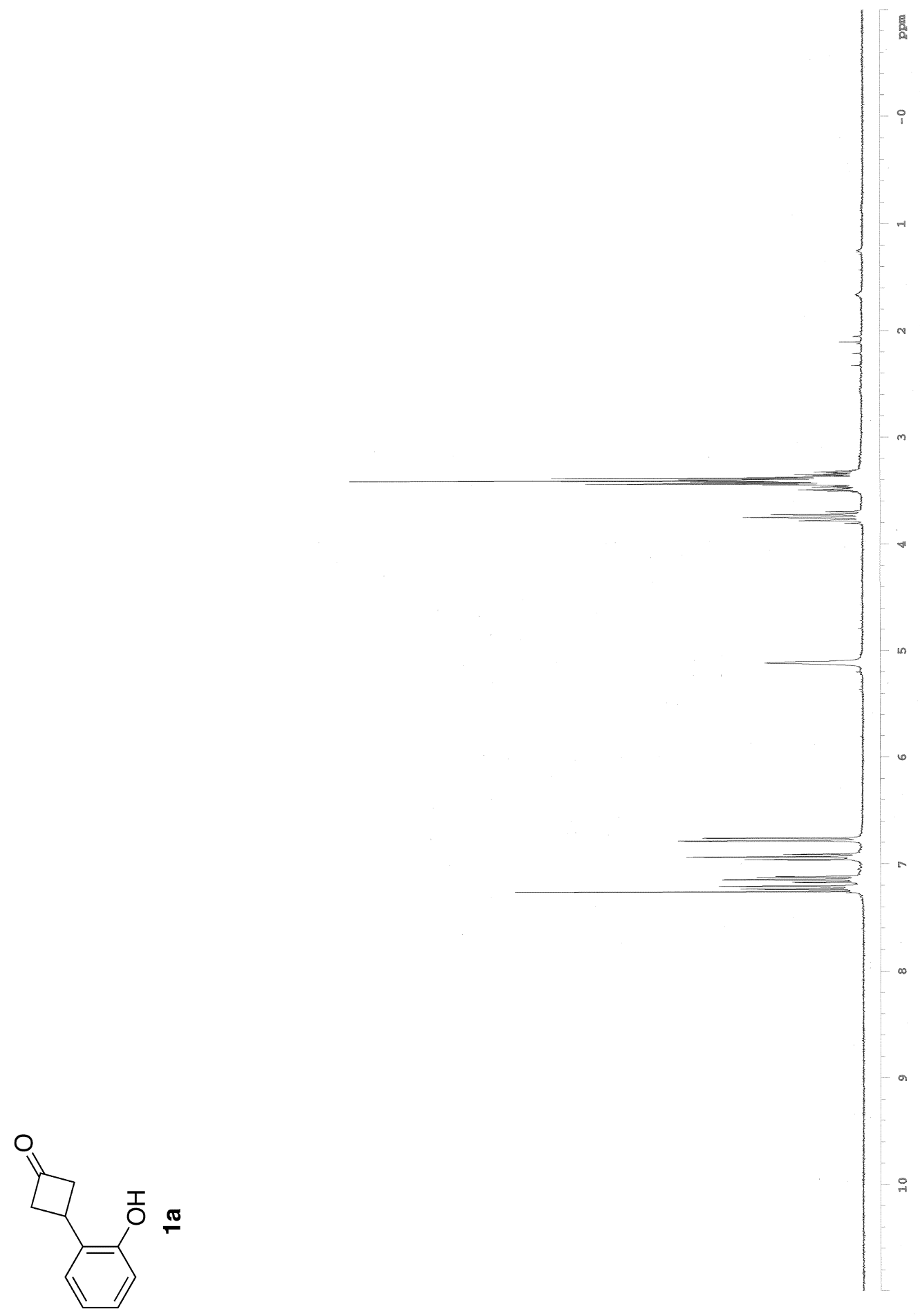




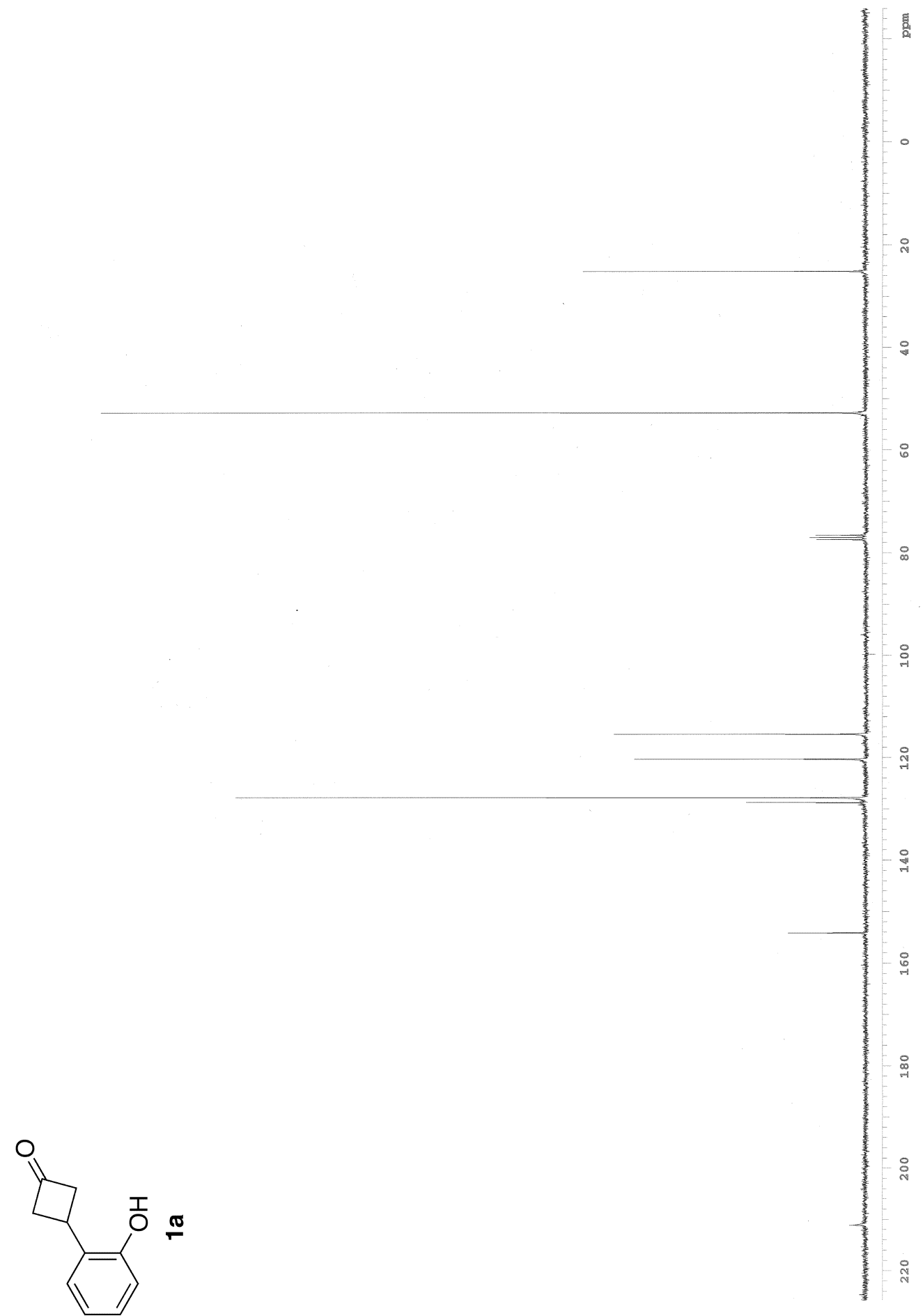




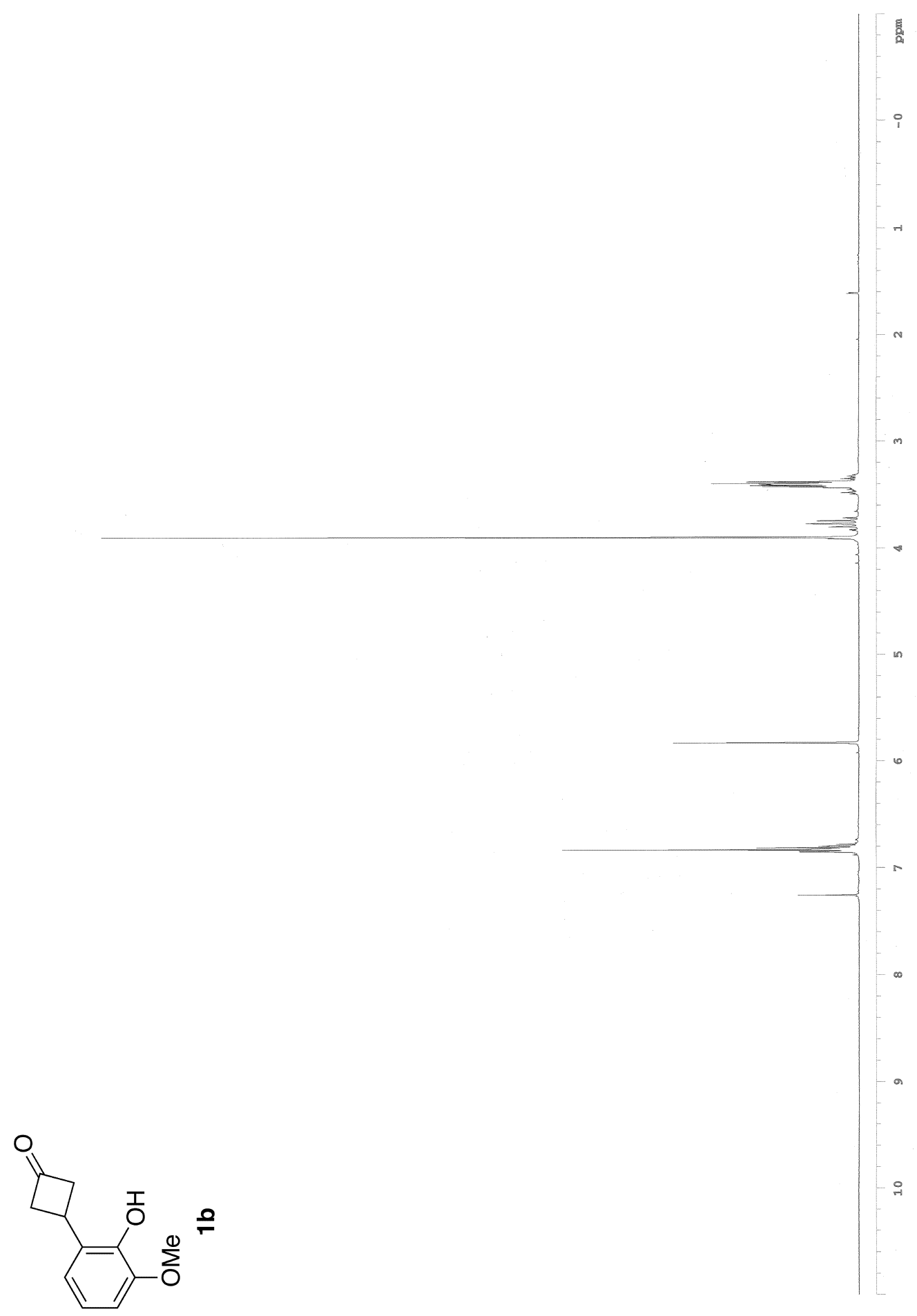




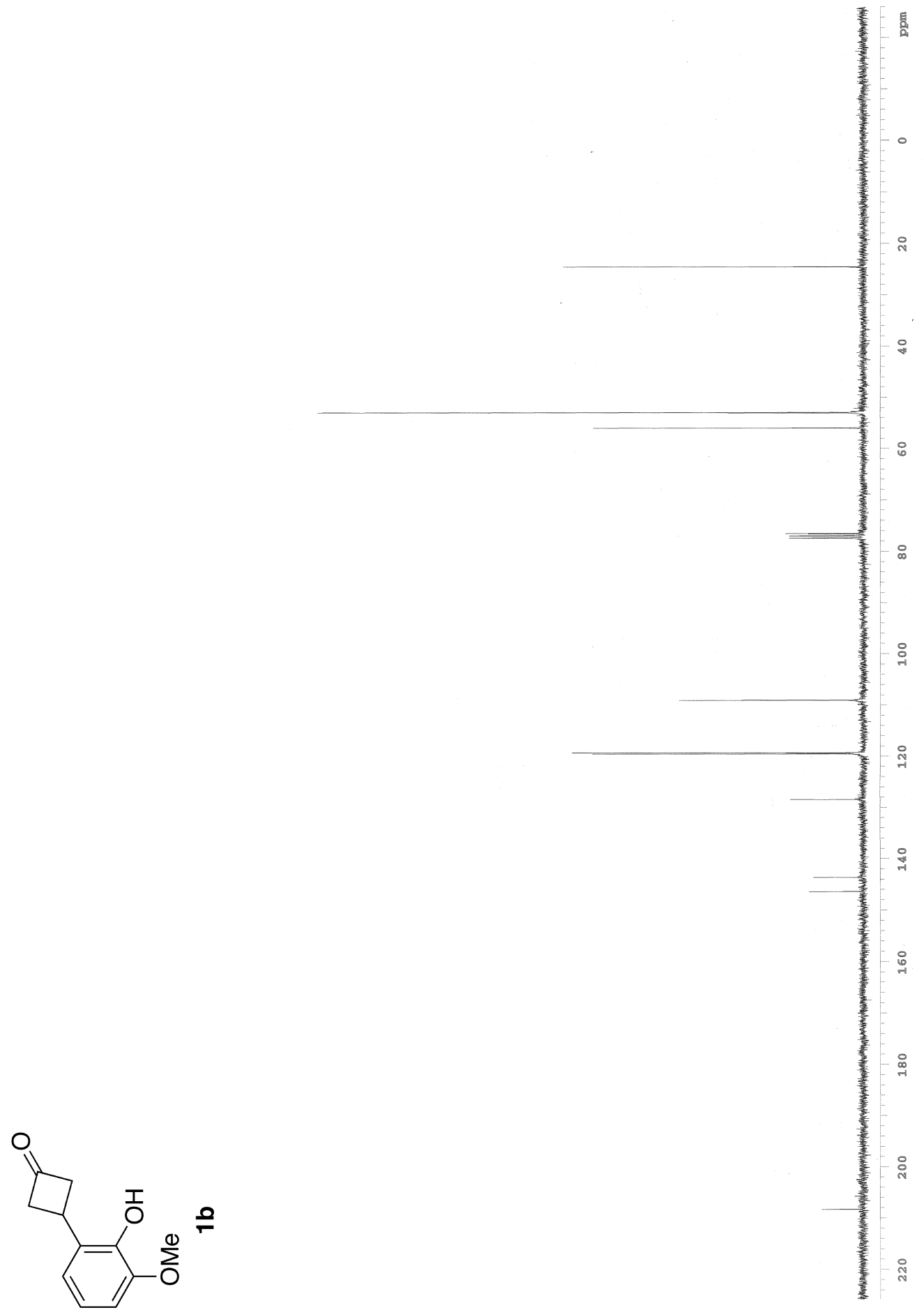




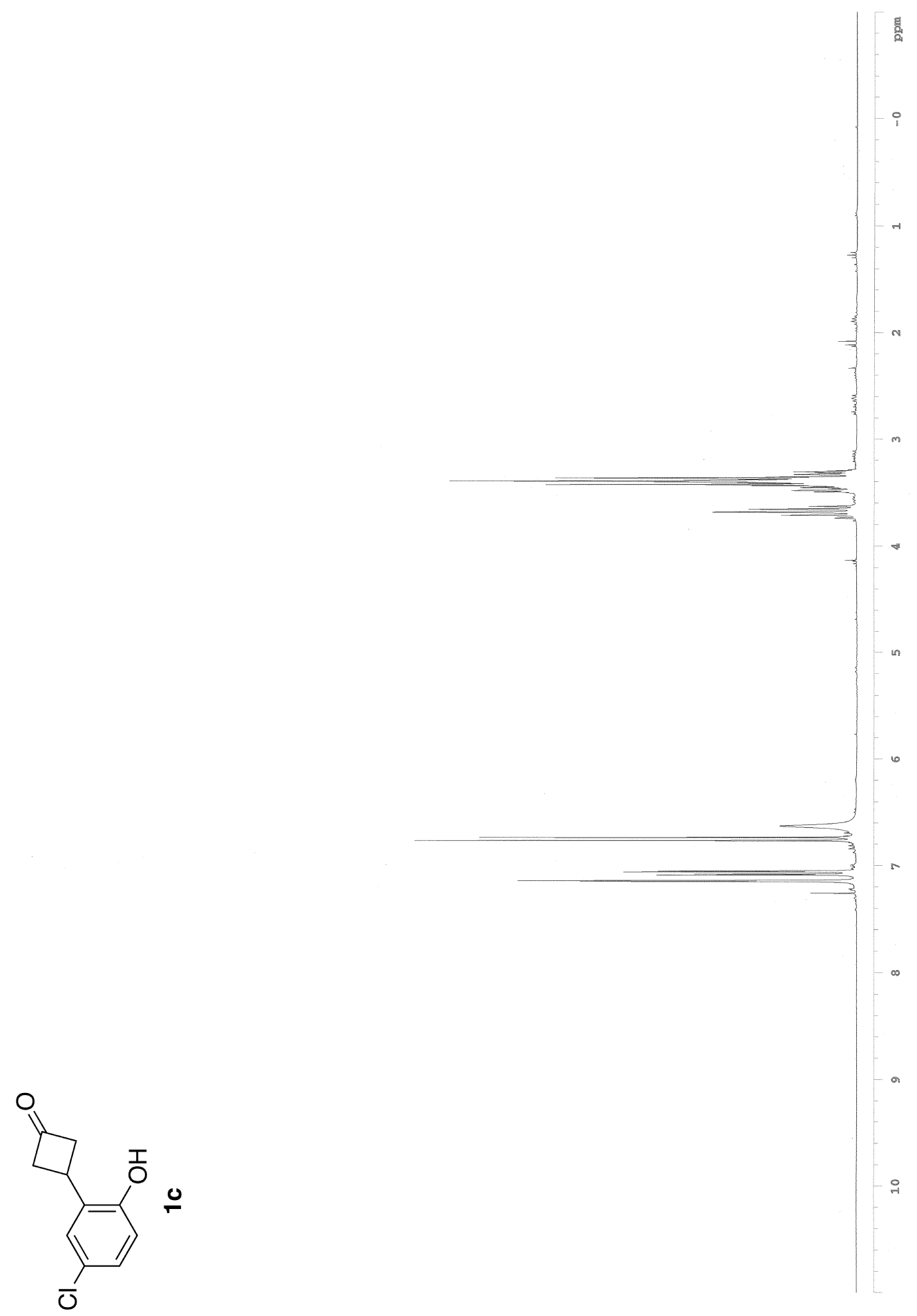




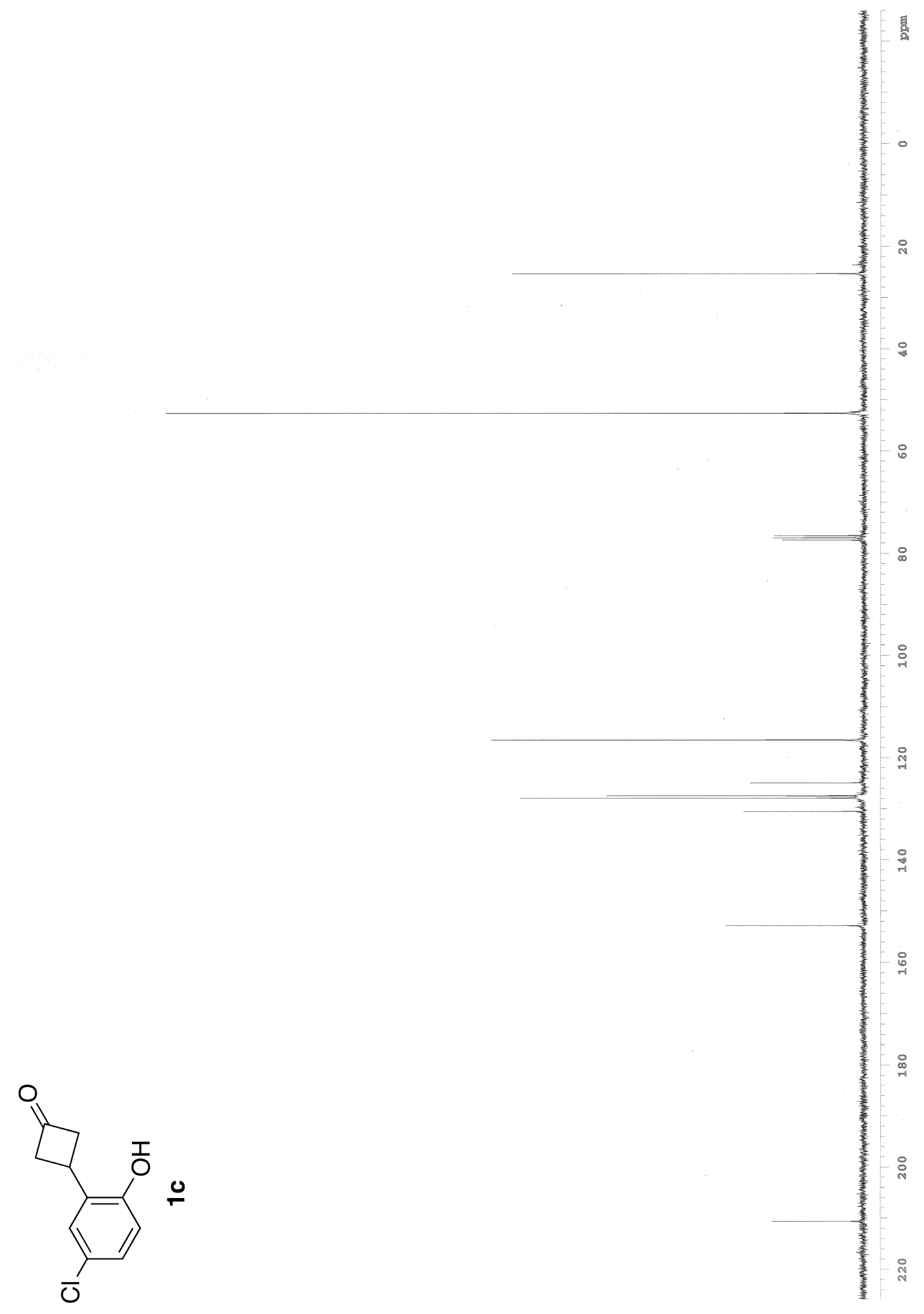




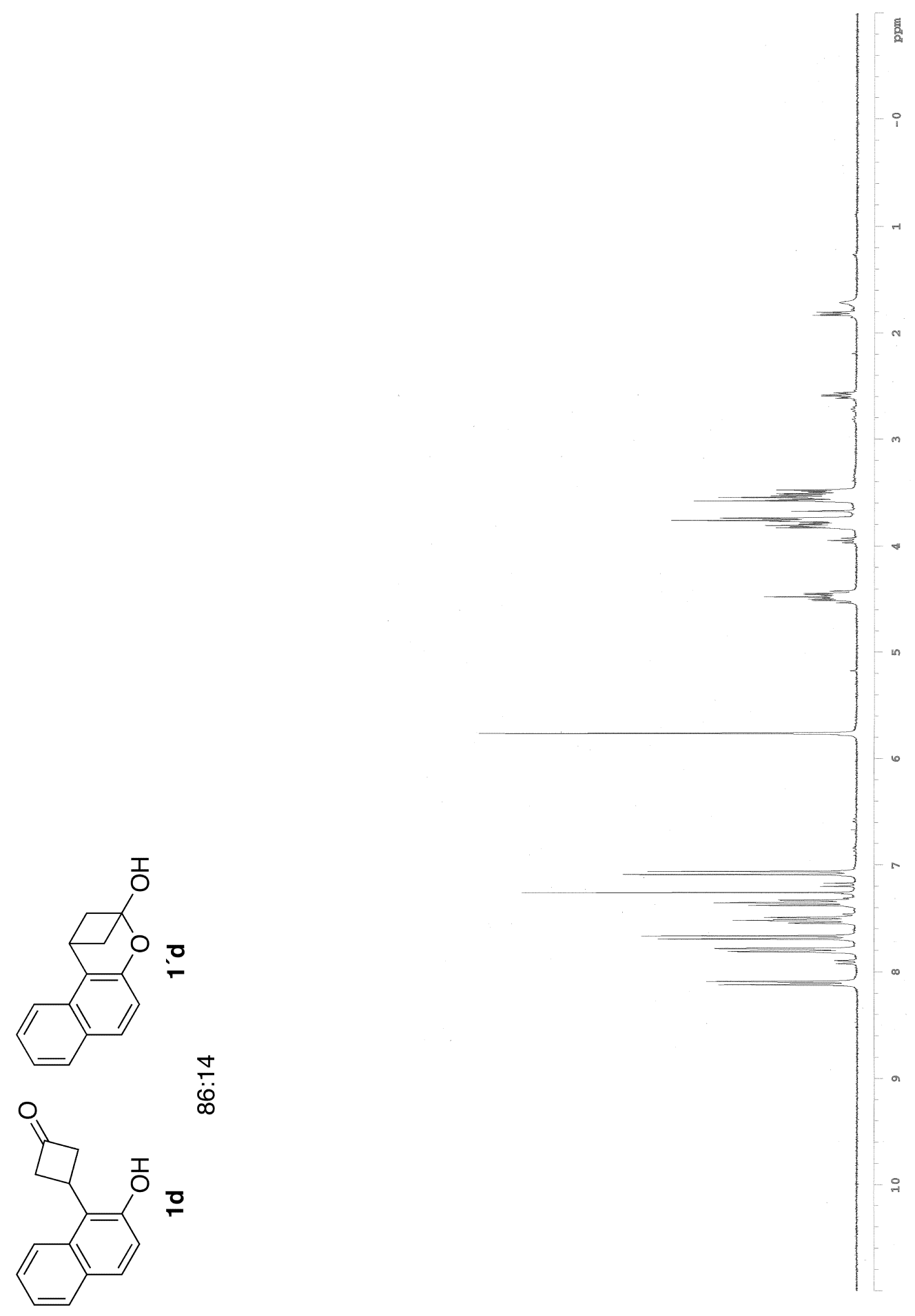




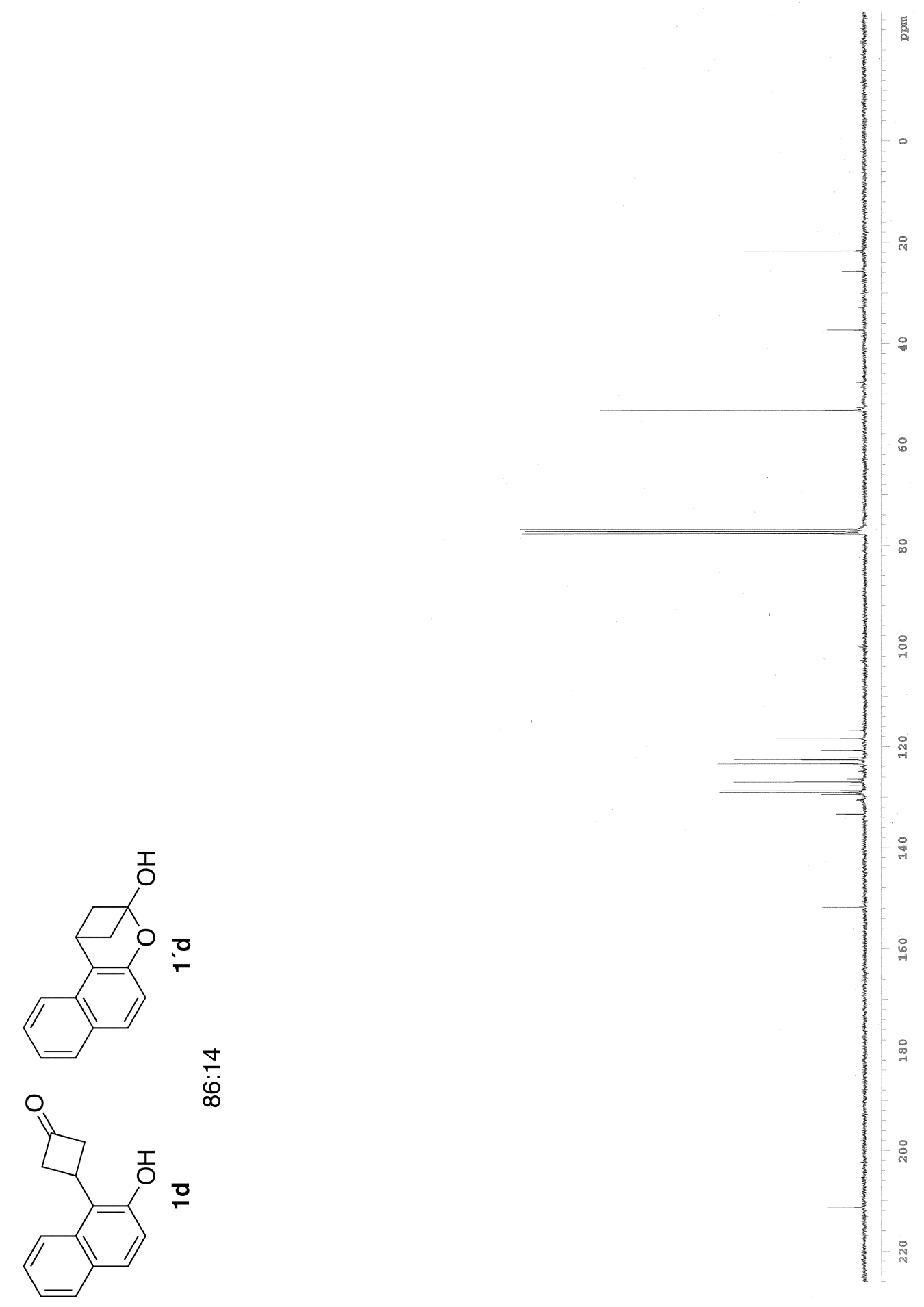




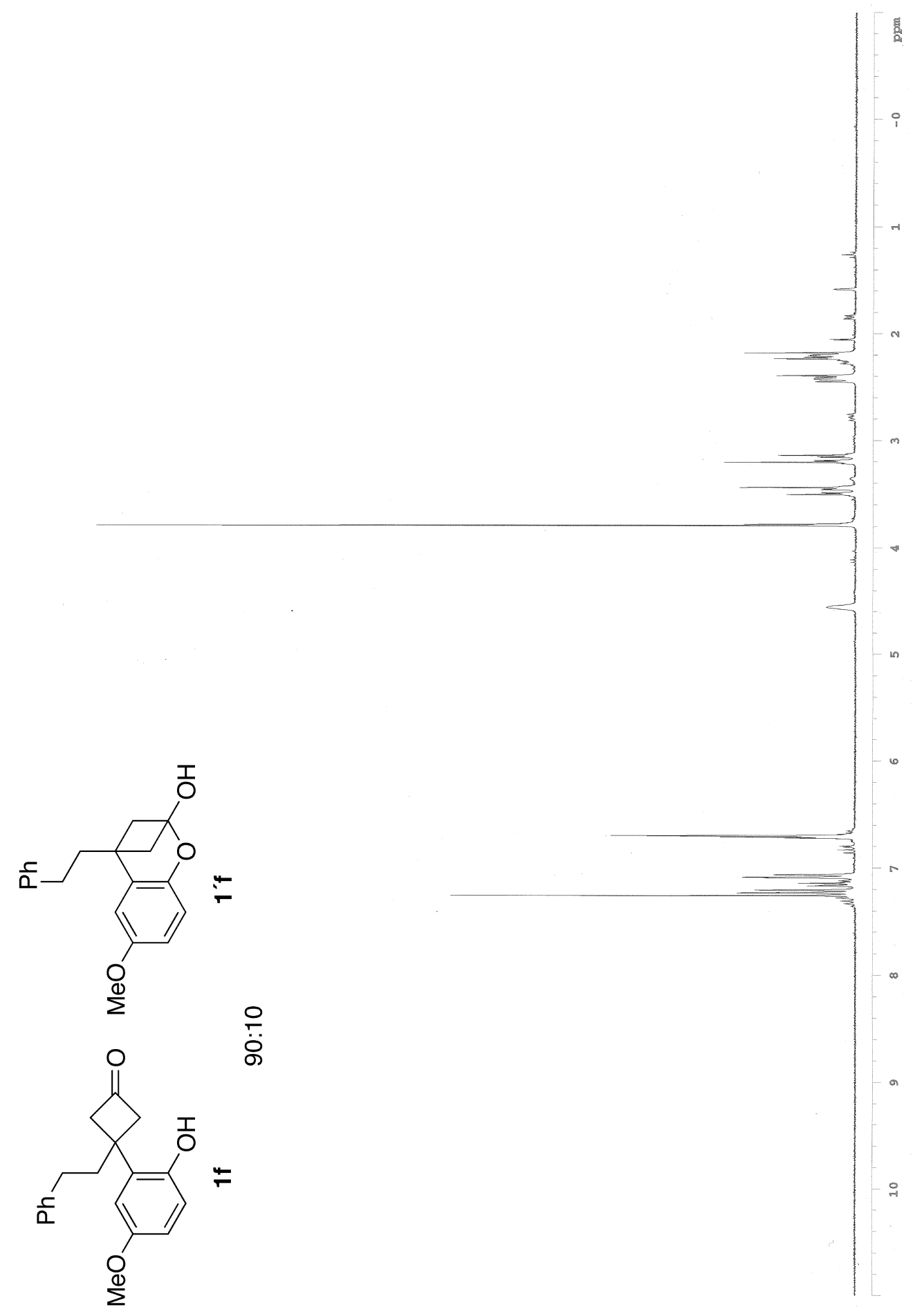



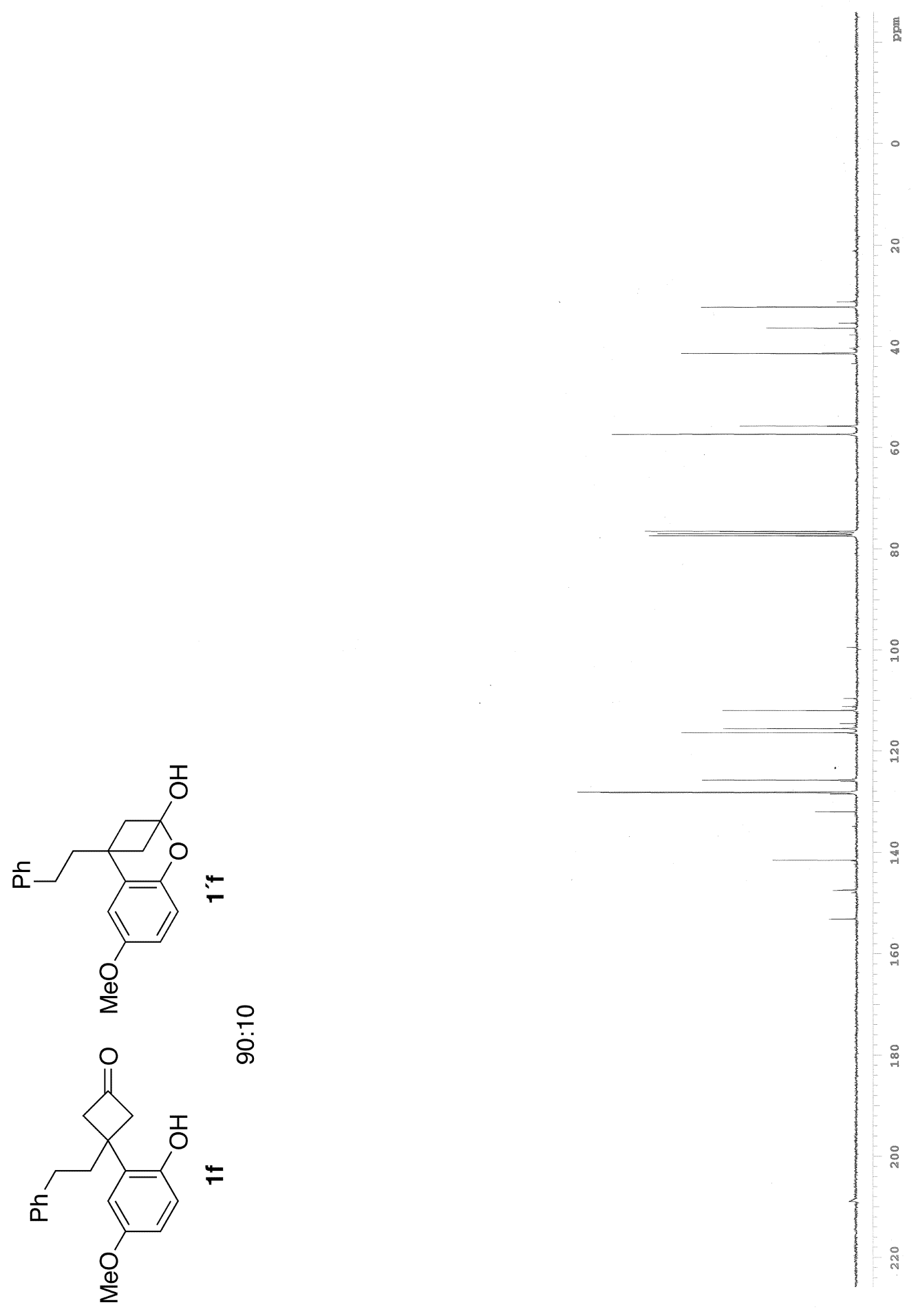


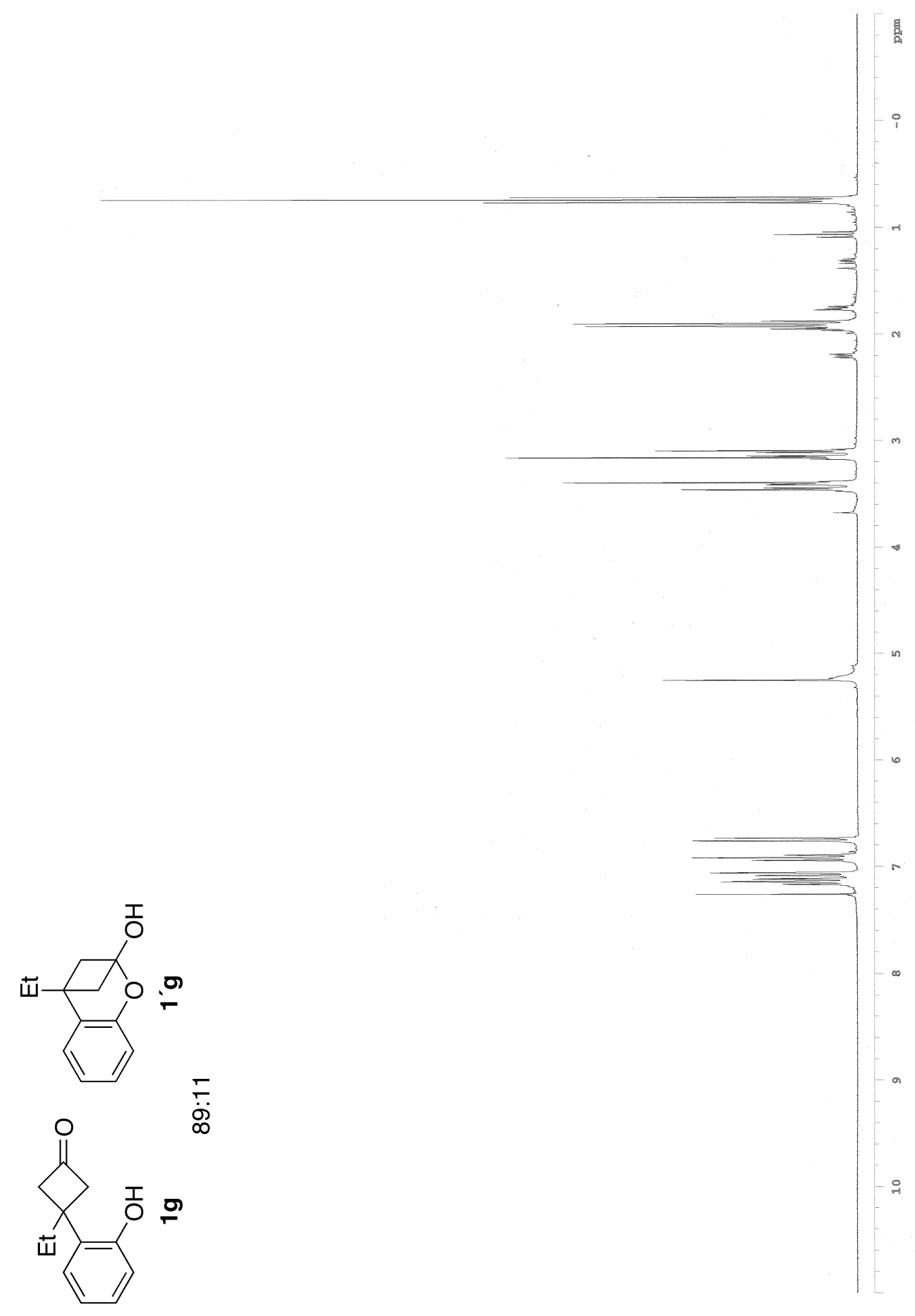




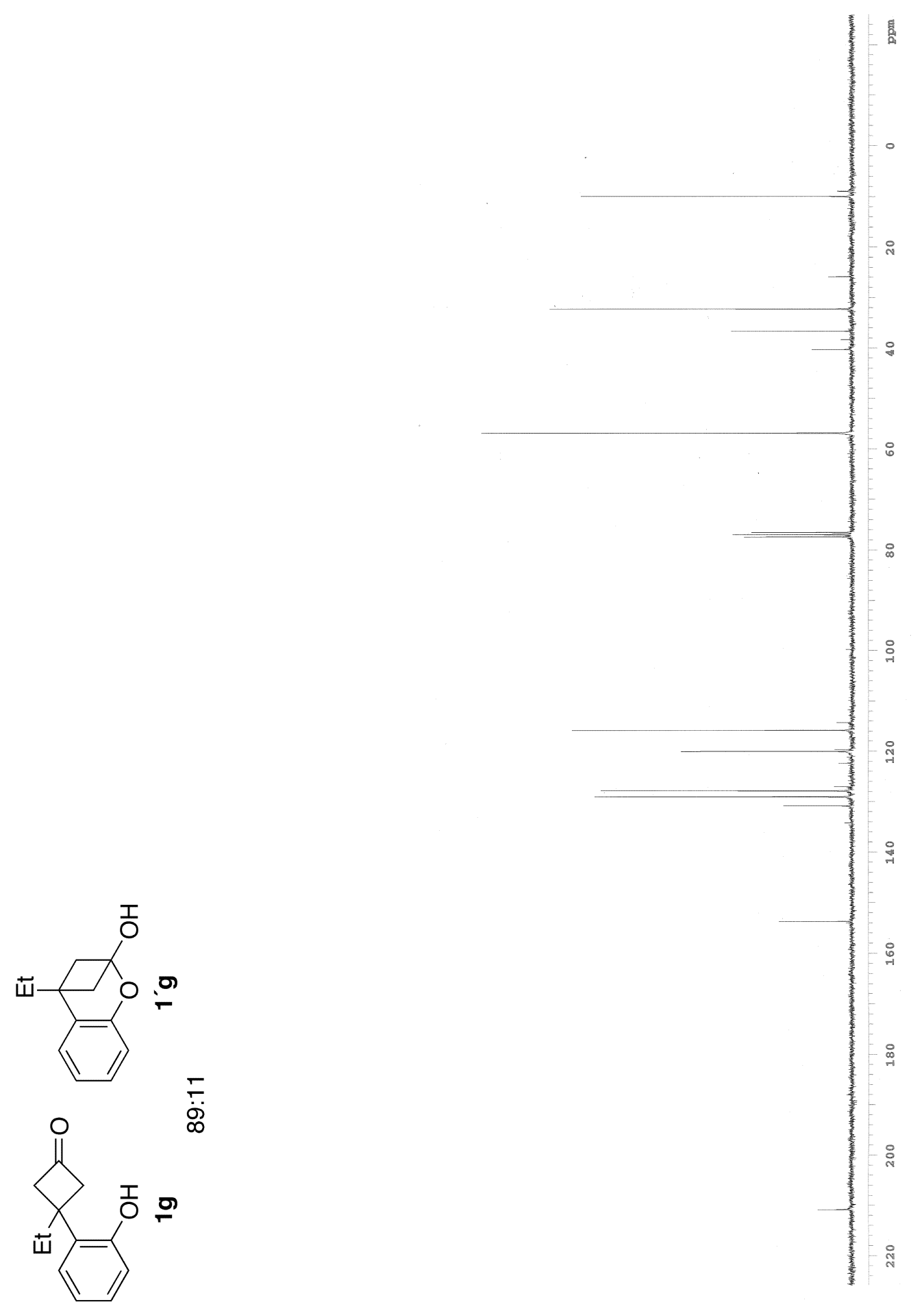




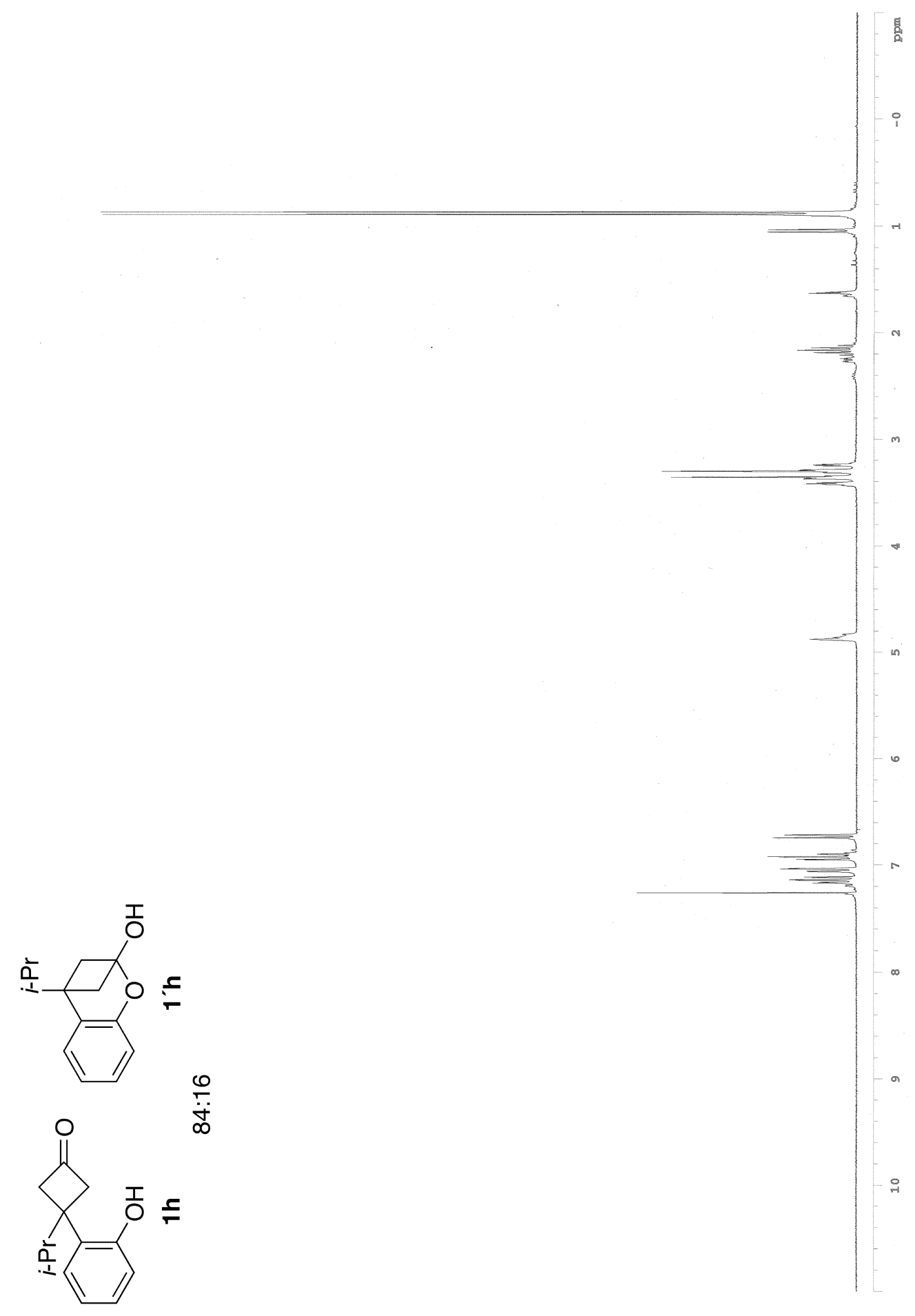




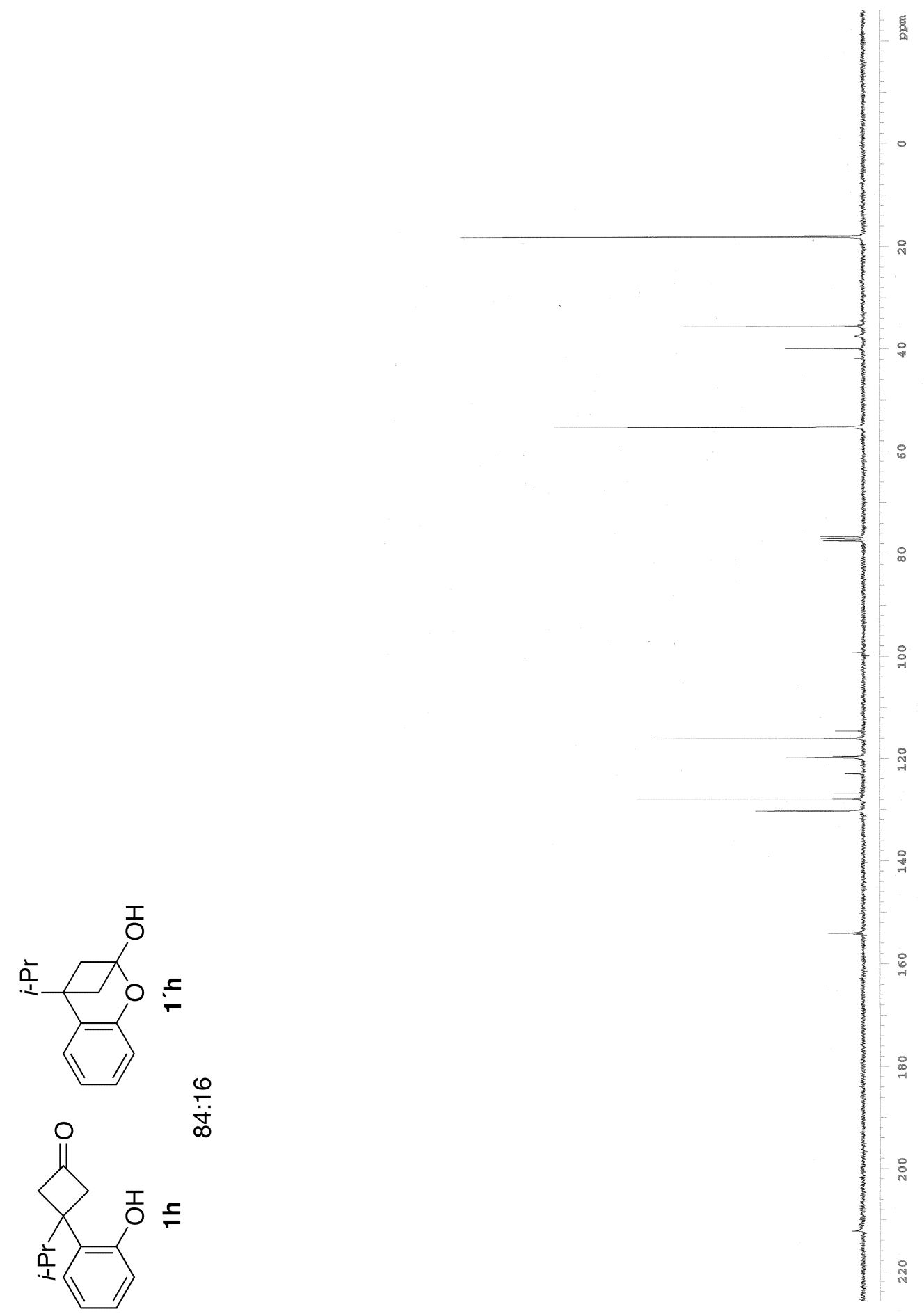




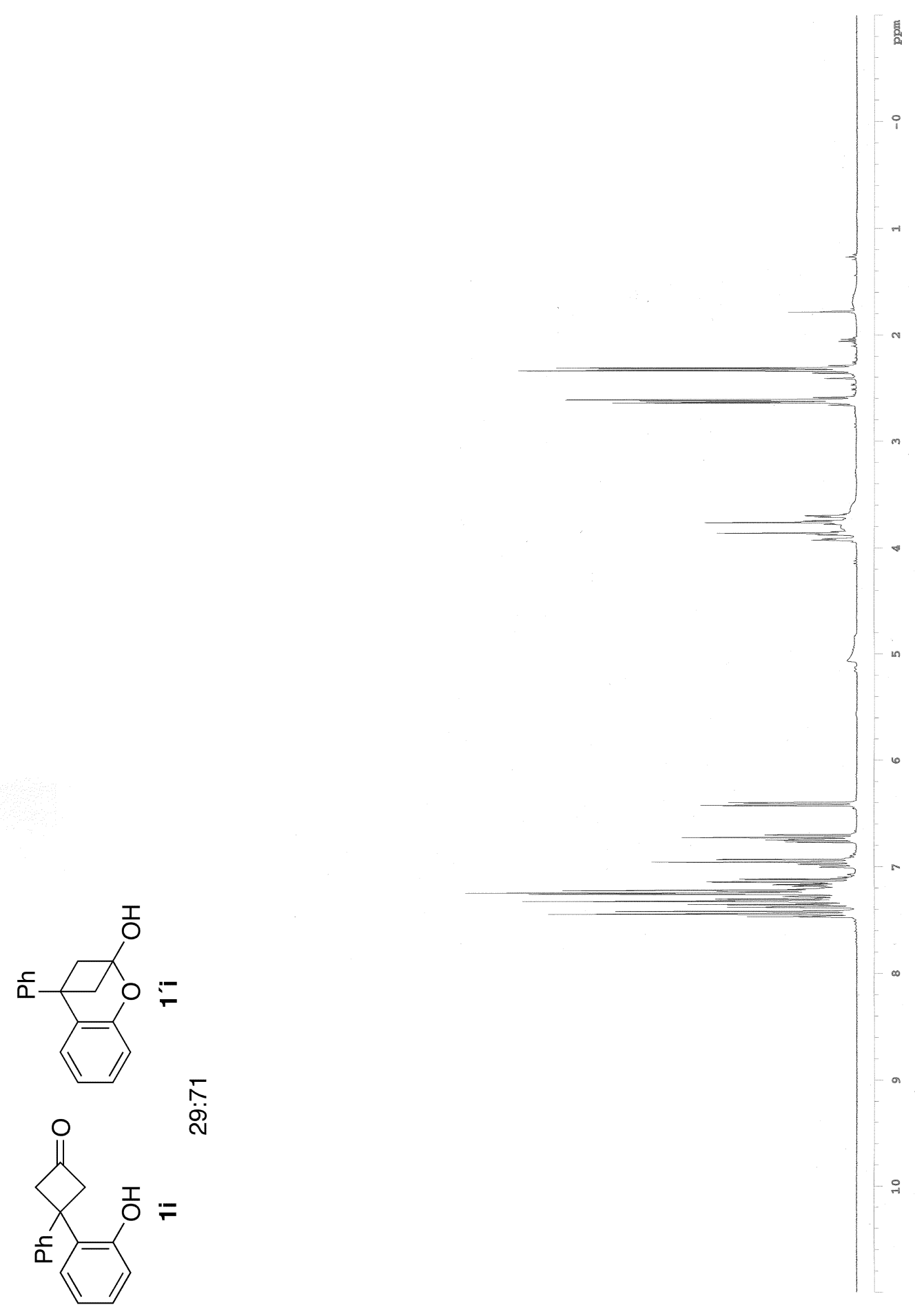




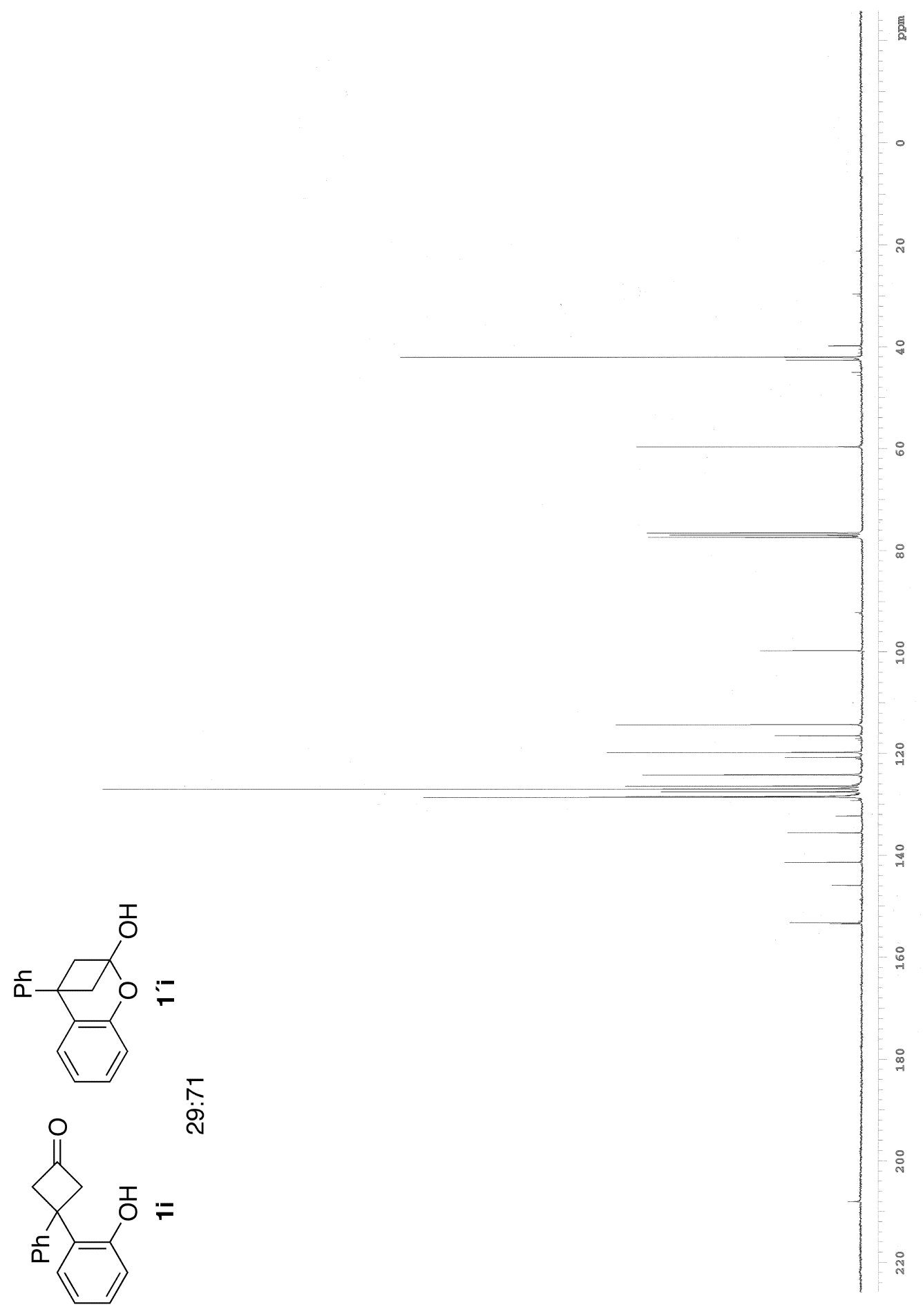




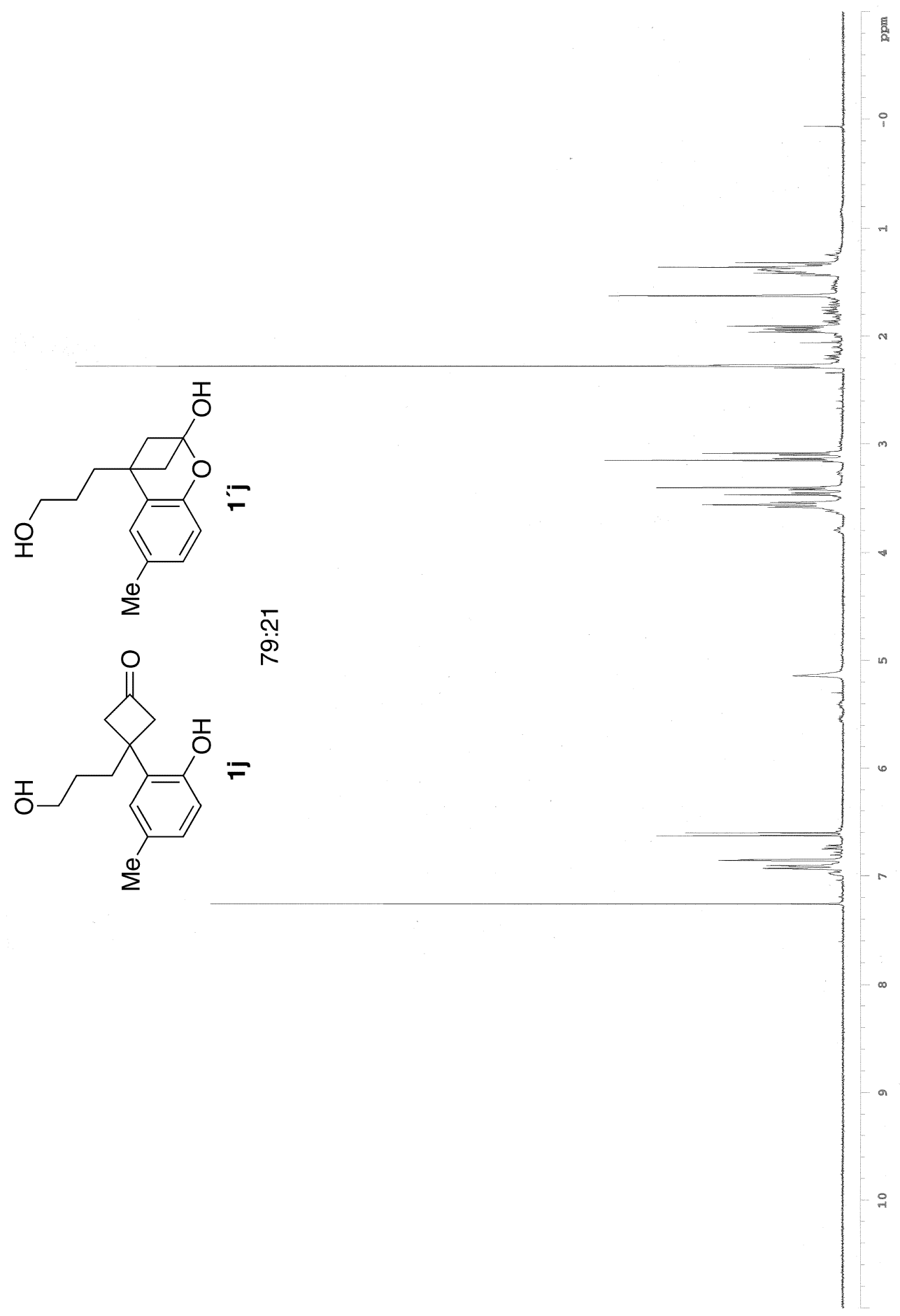




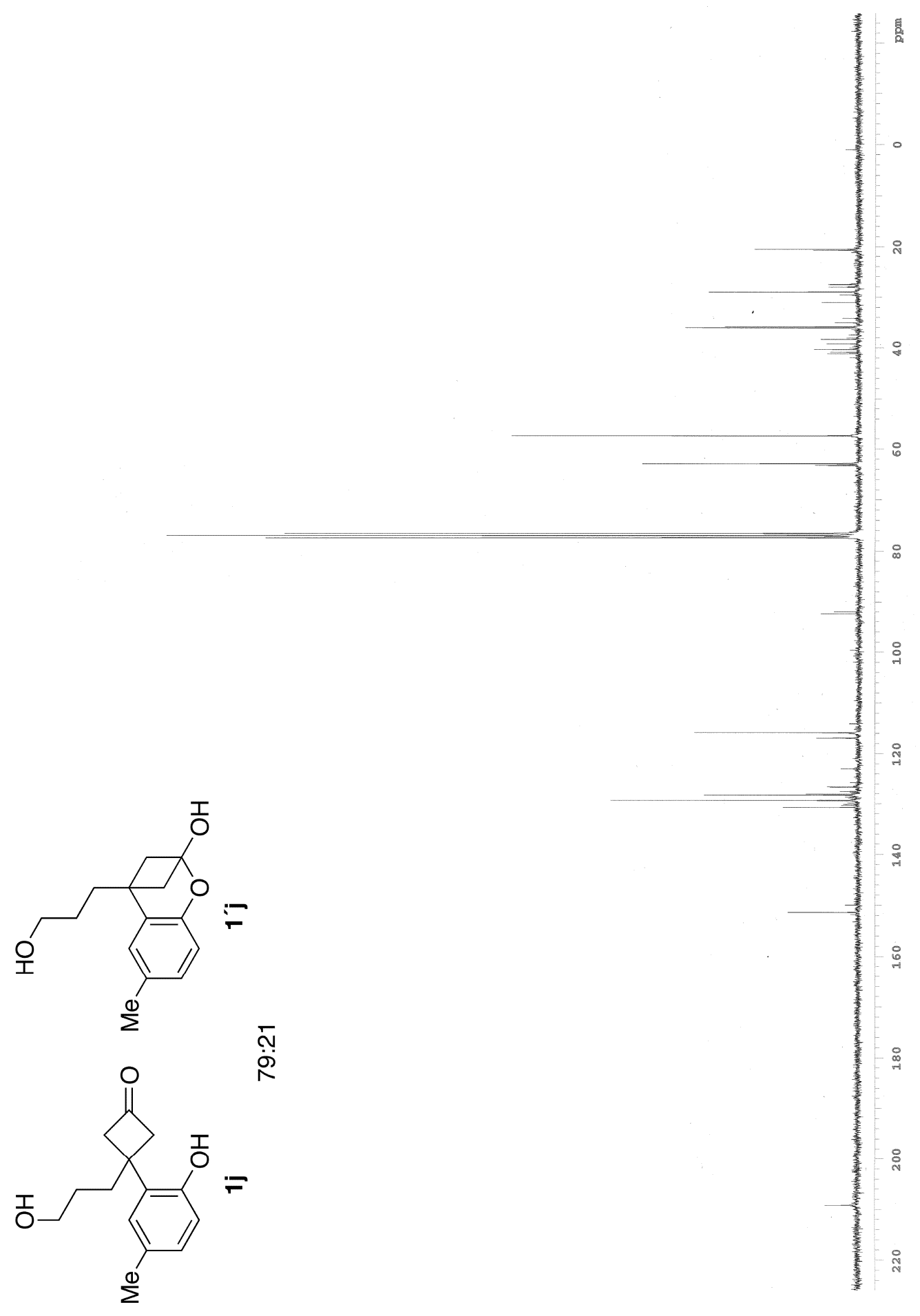




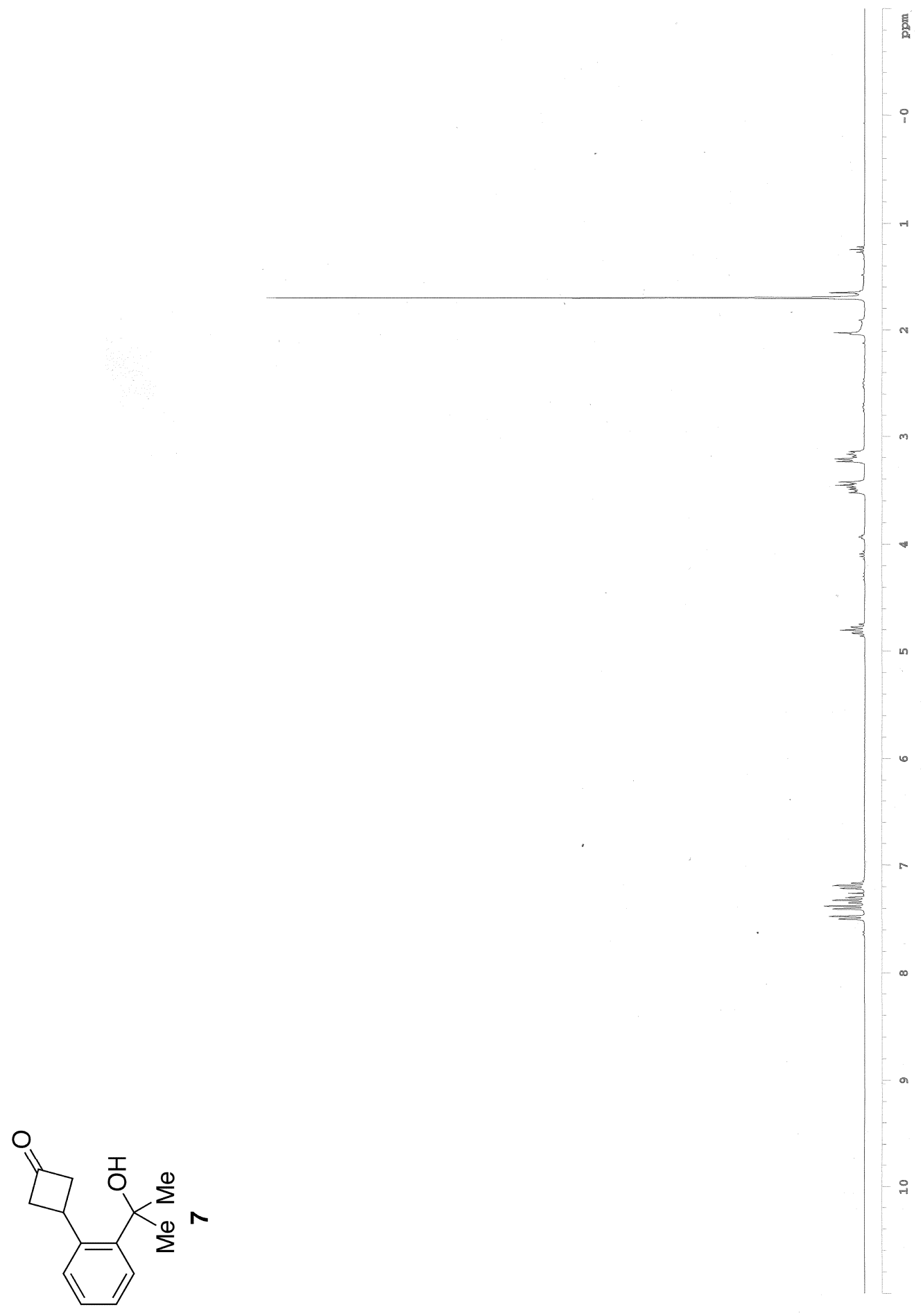




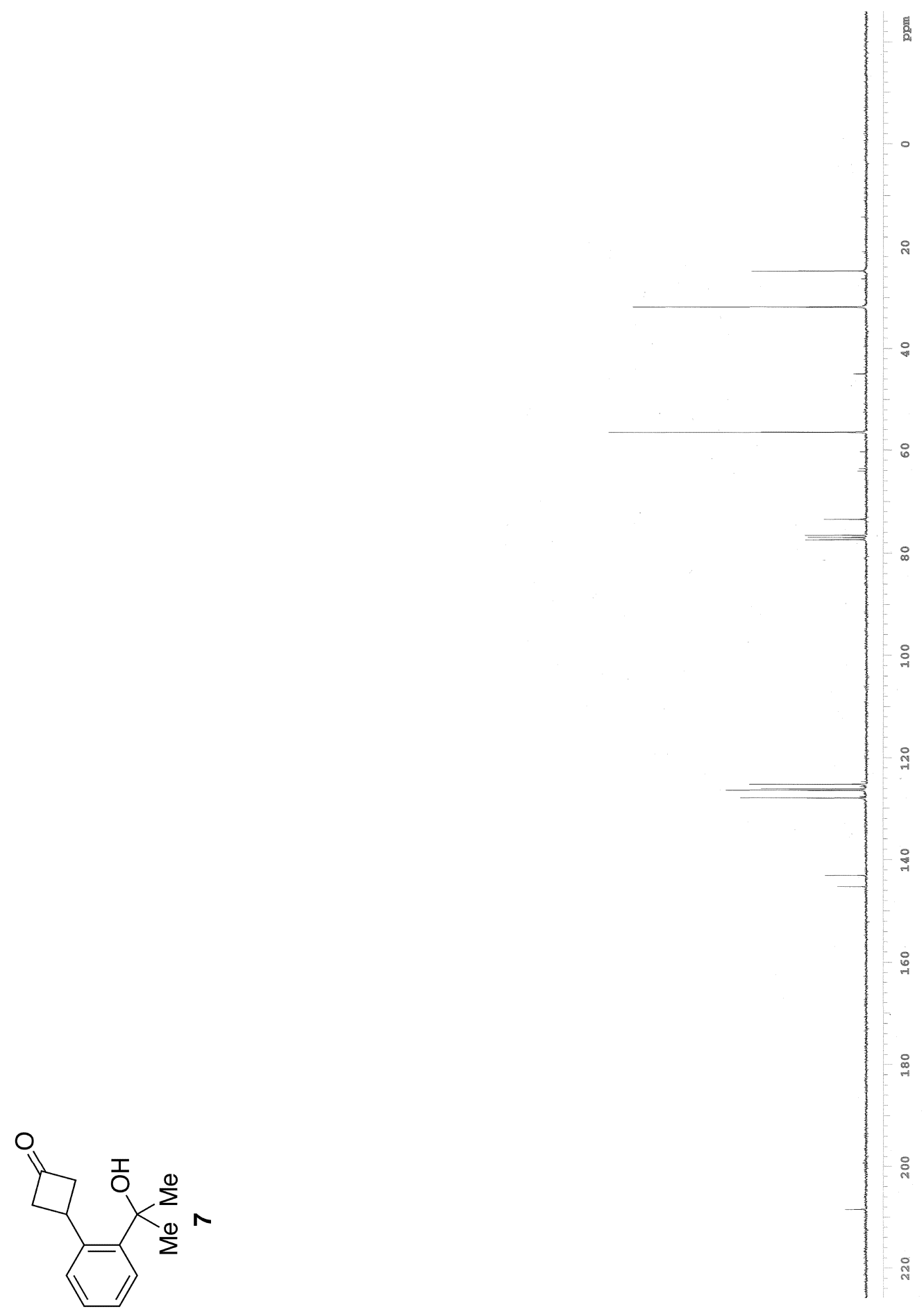




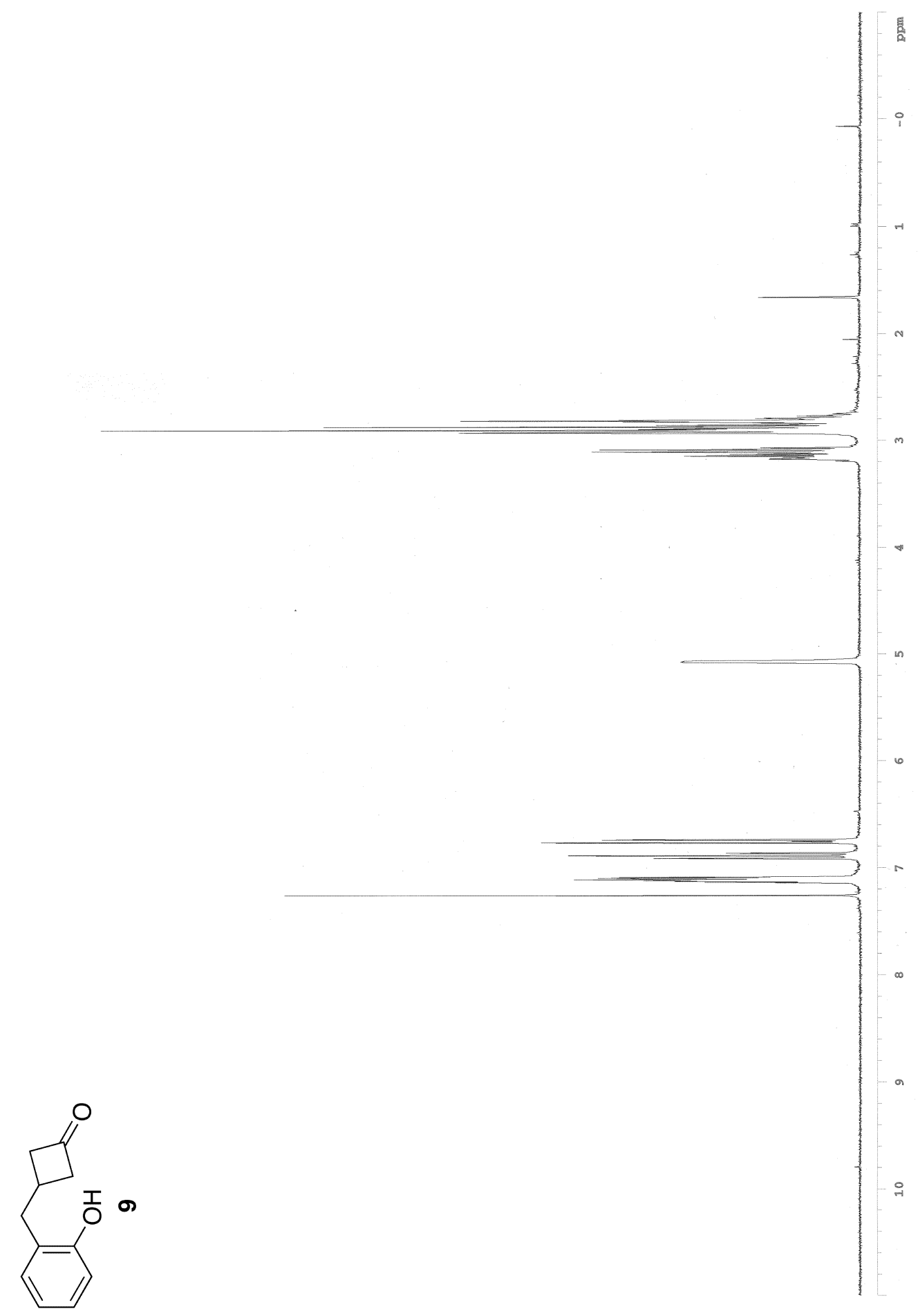




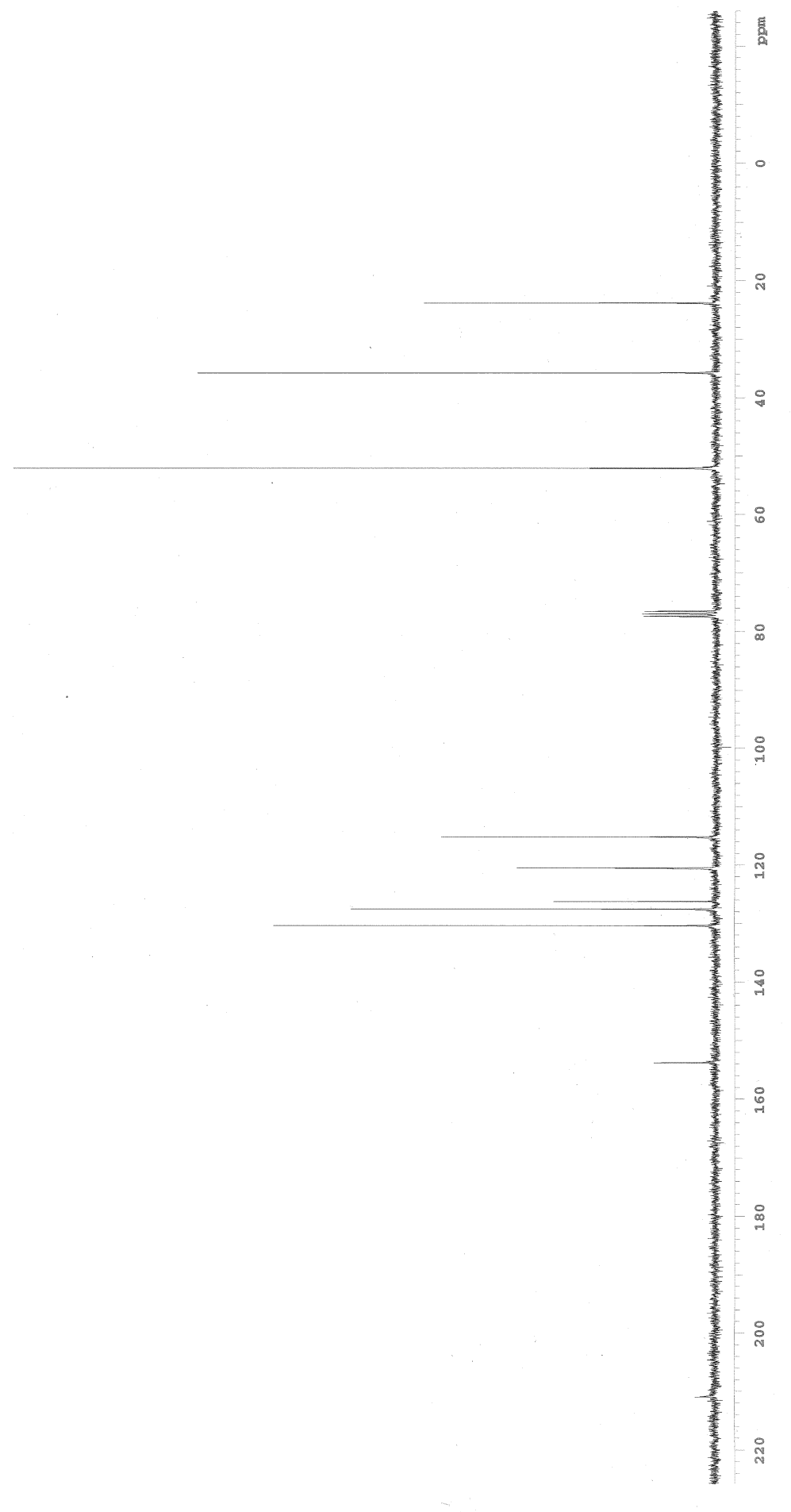

S44 


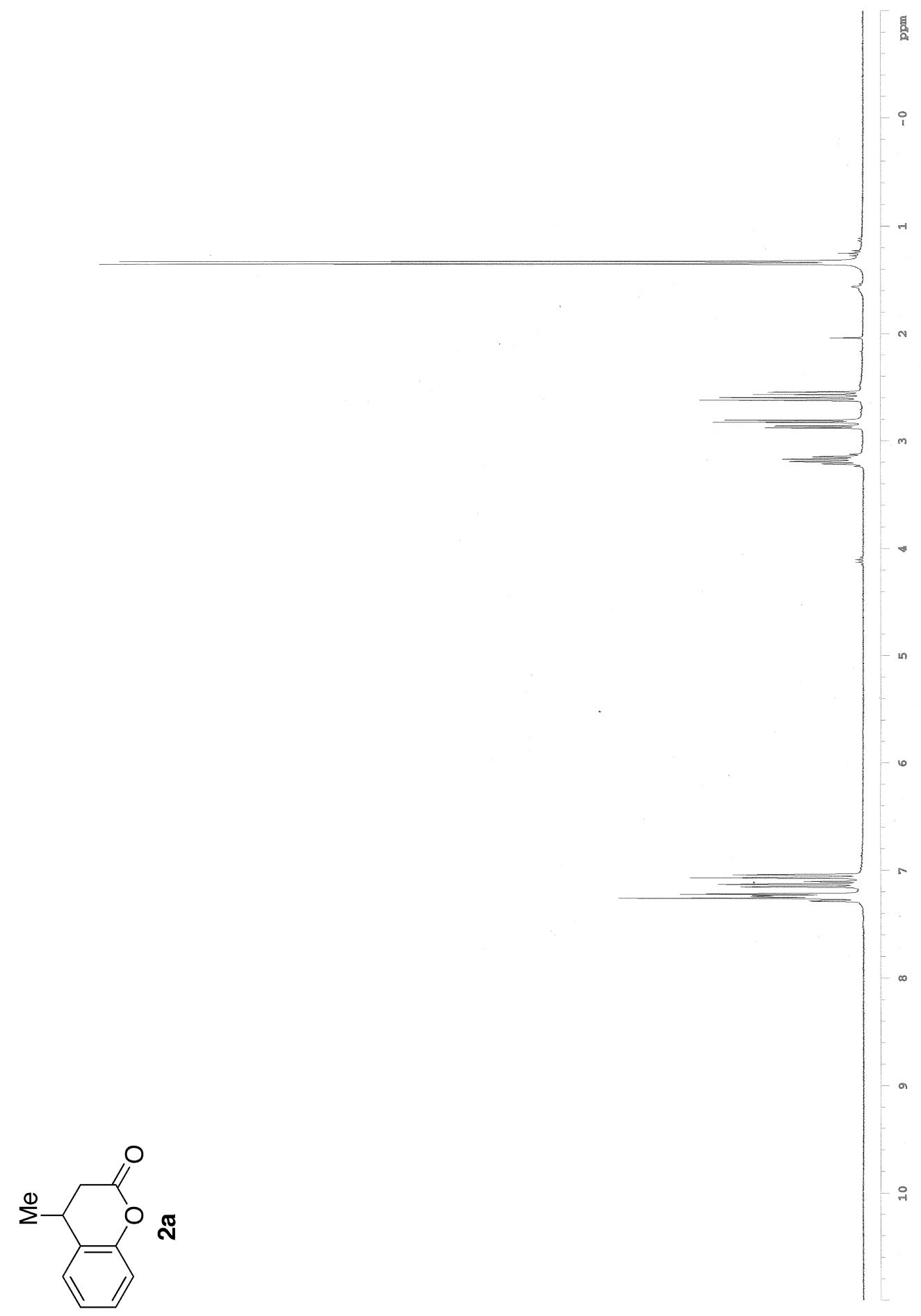




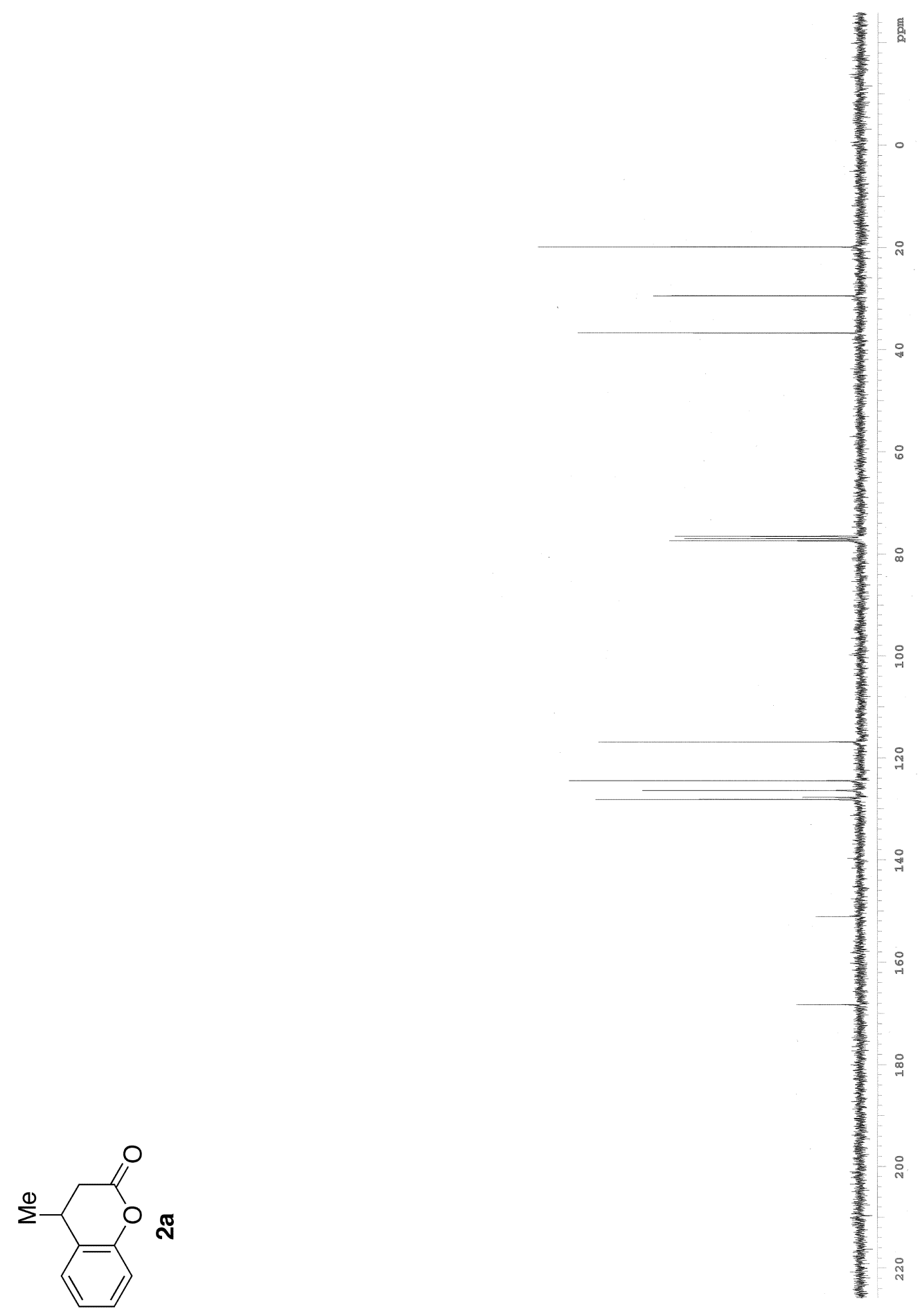




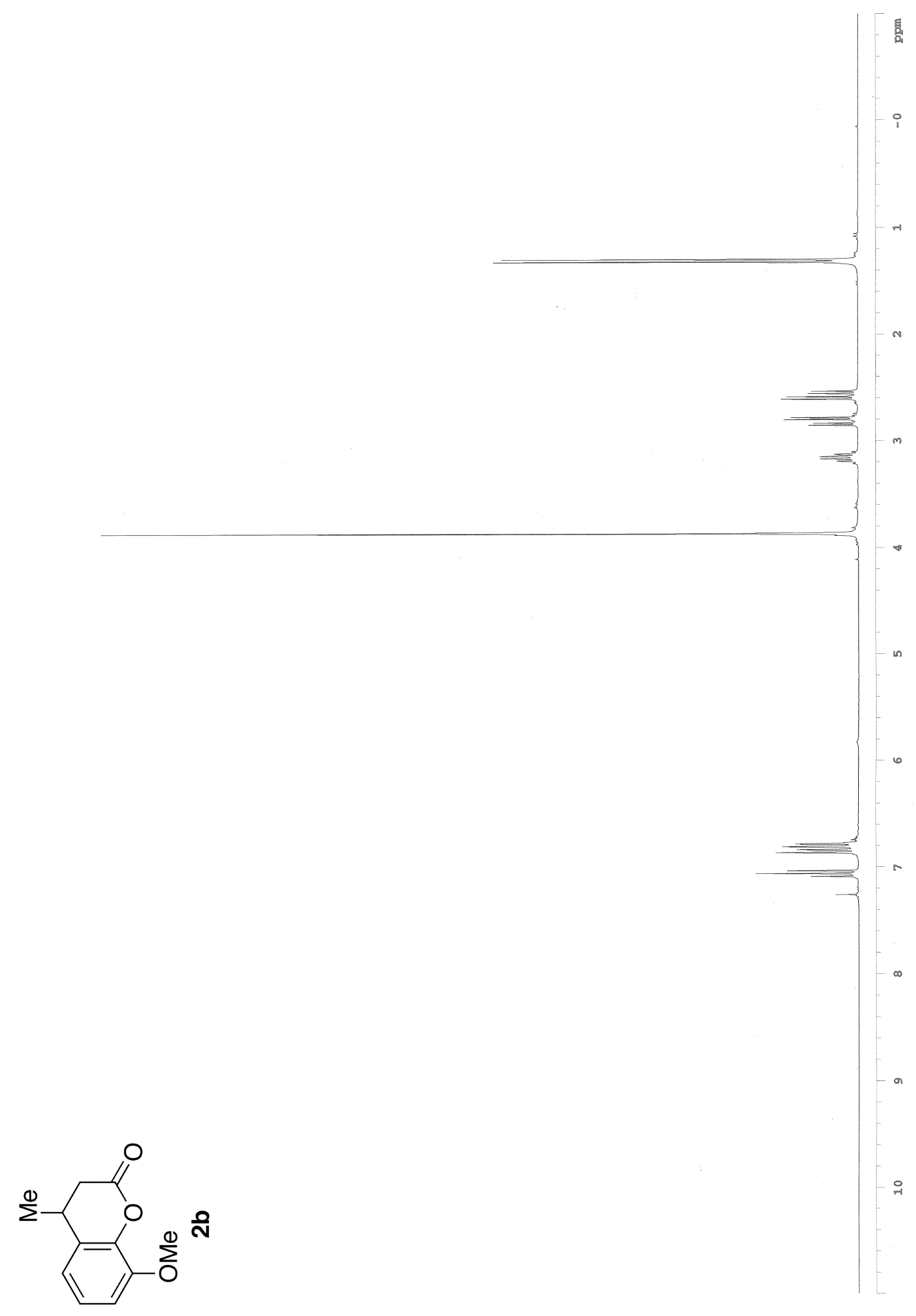




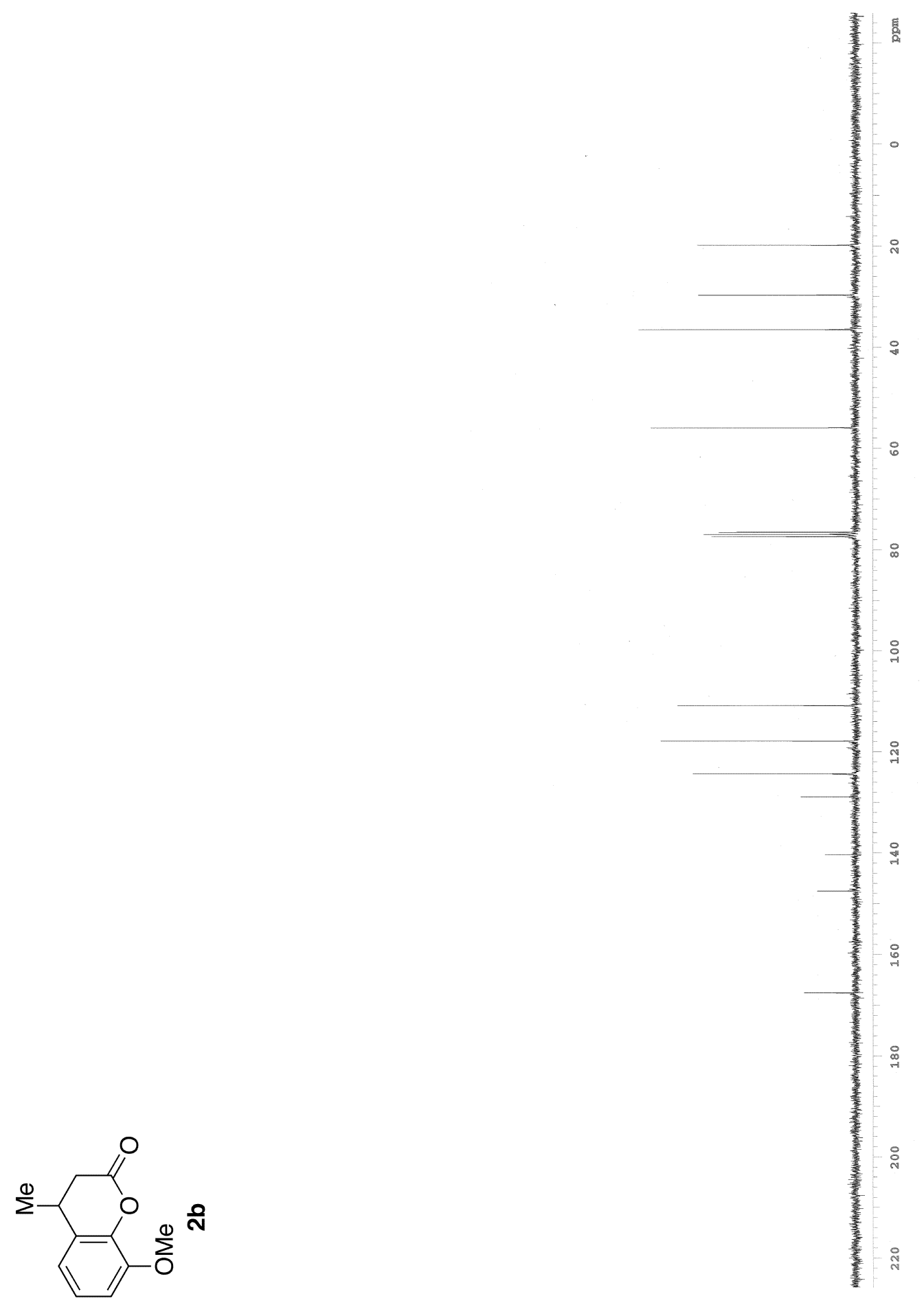




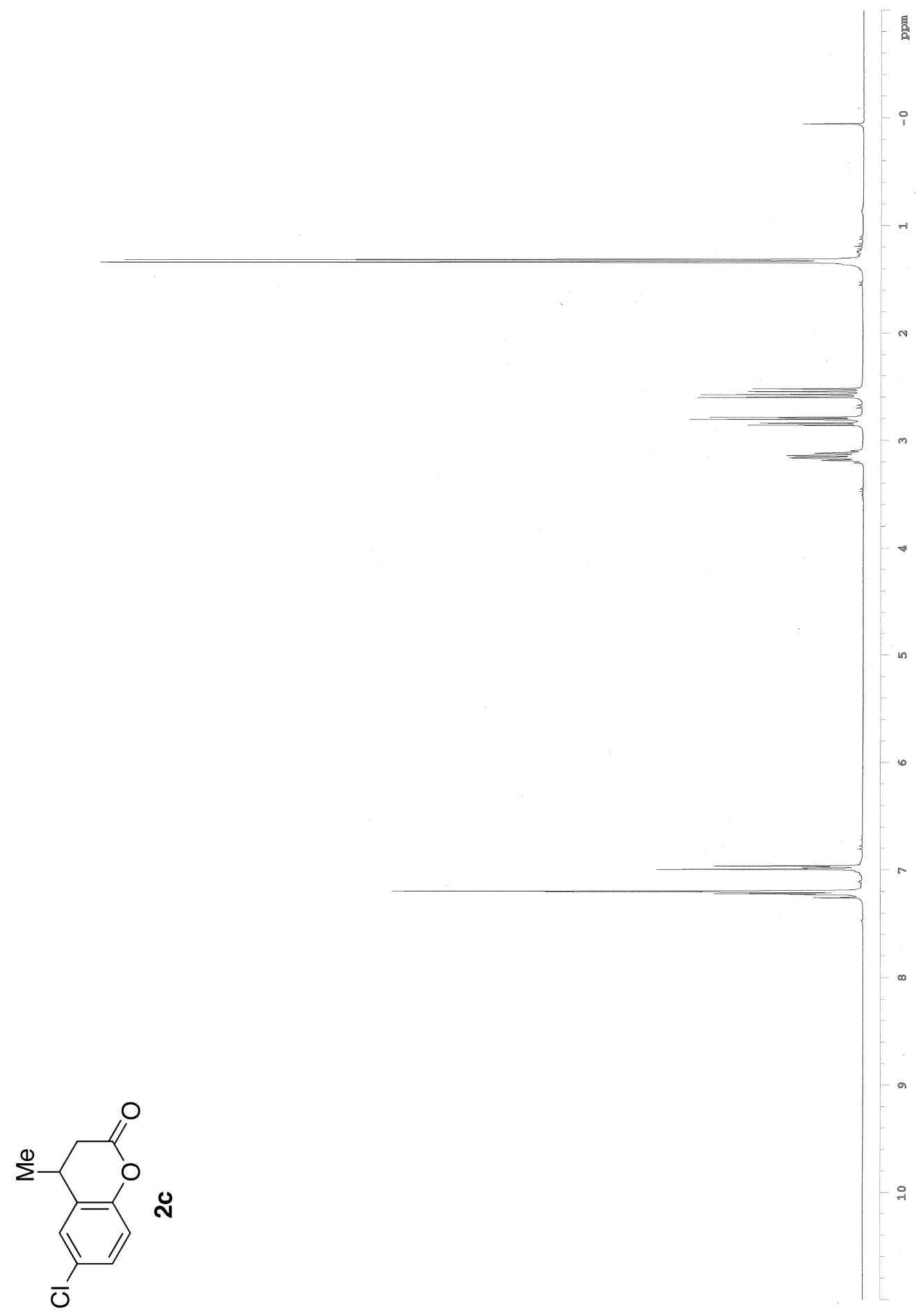




$$
\text { . }
$$




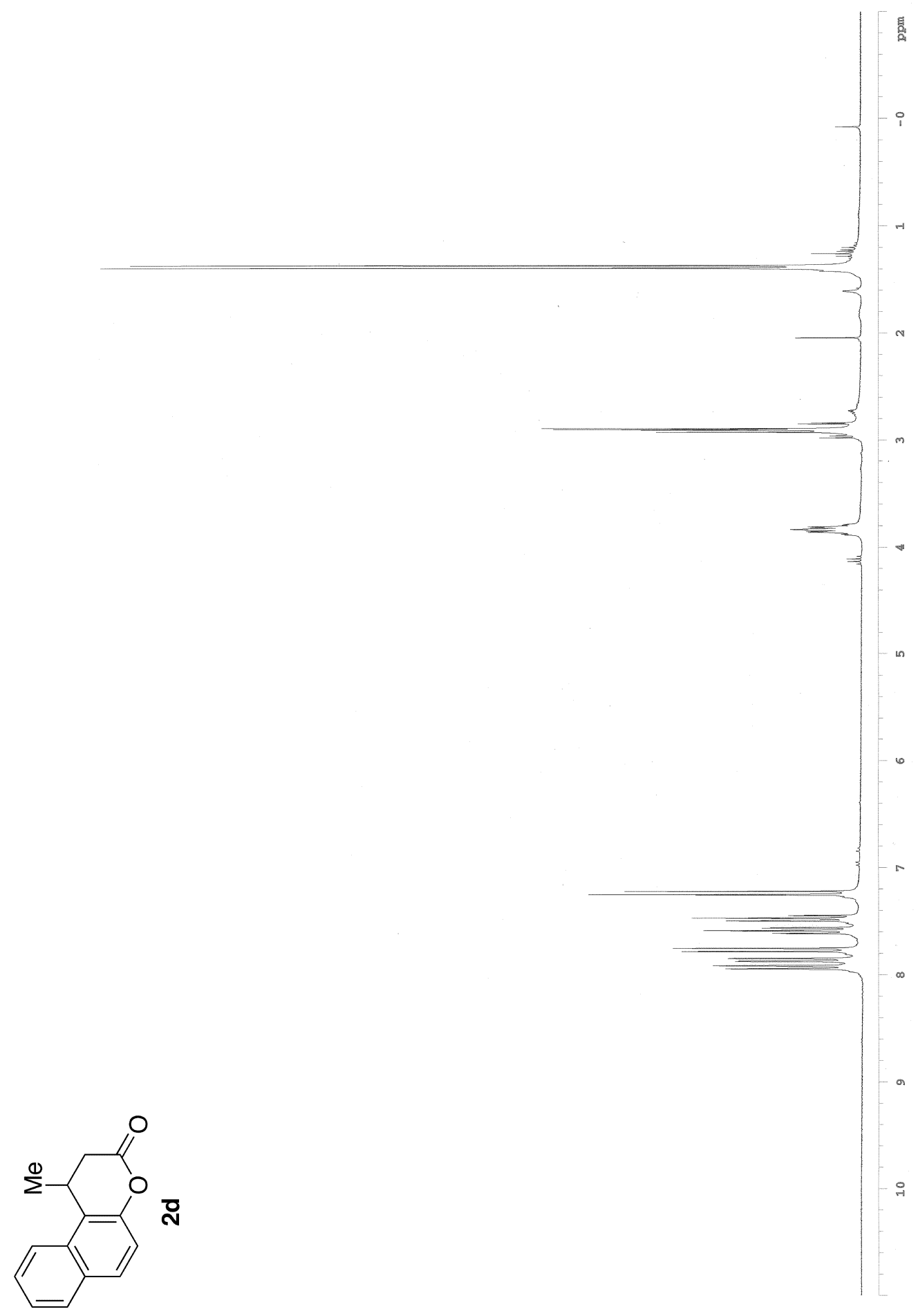




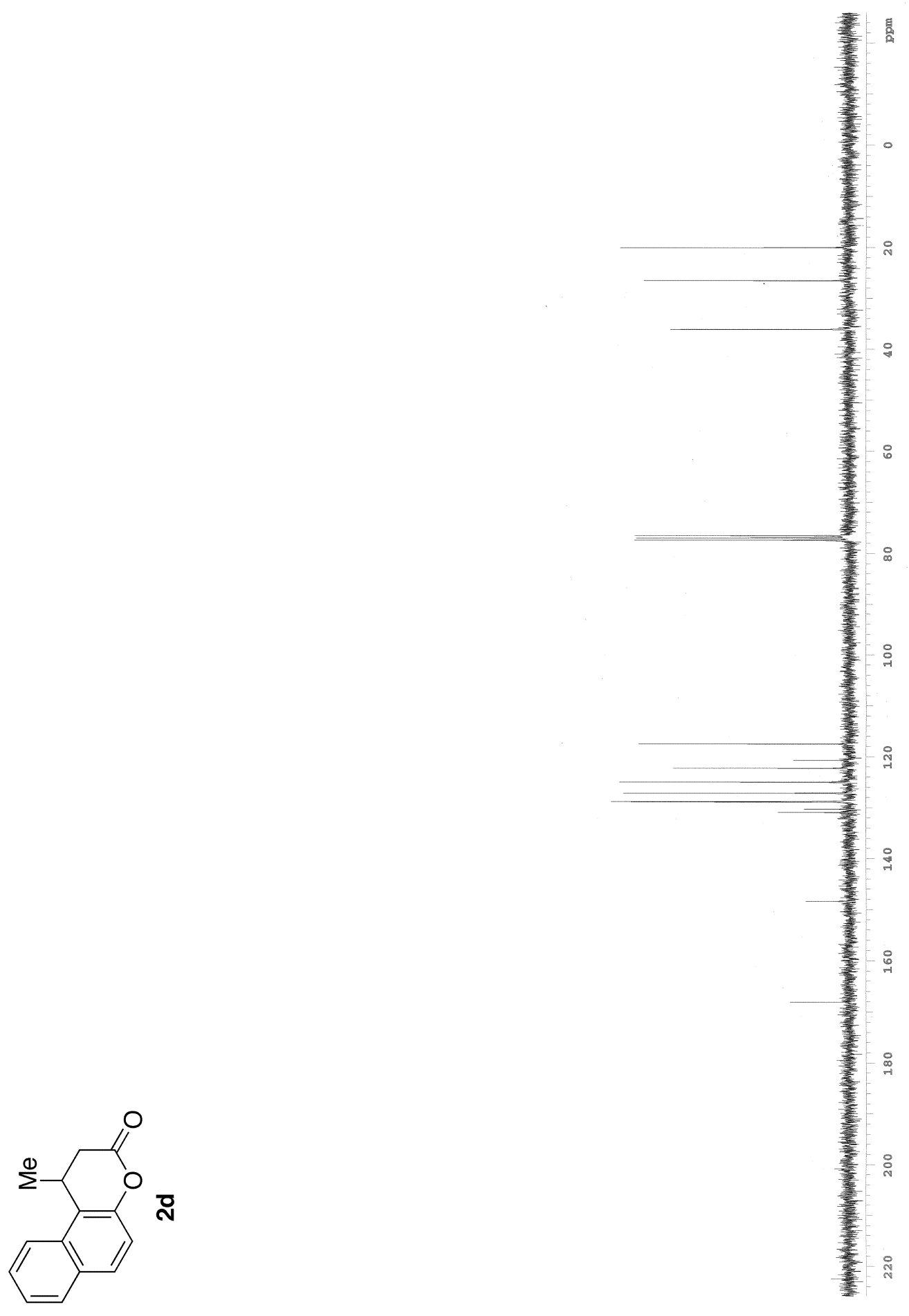




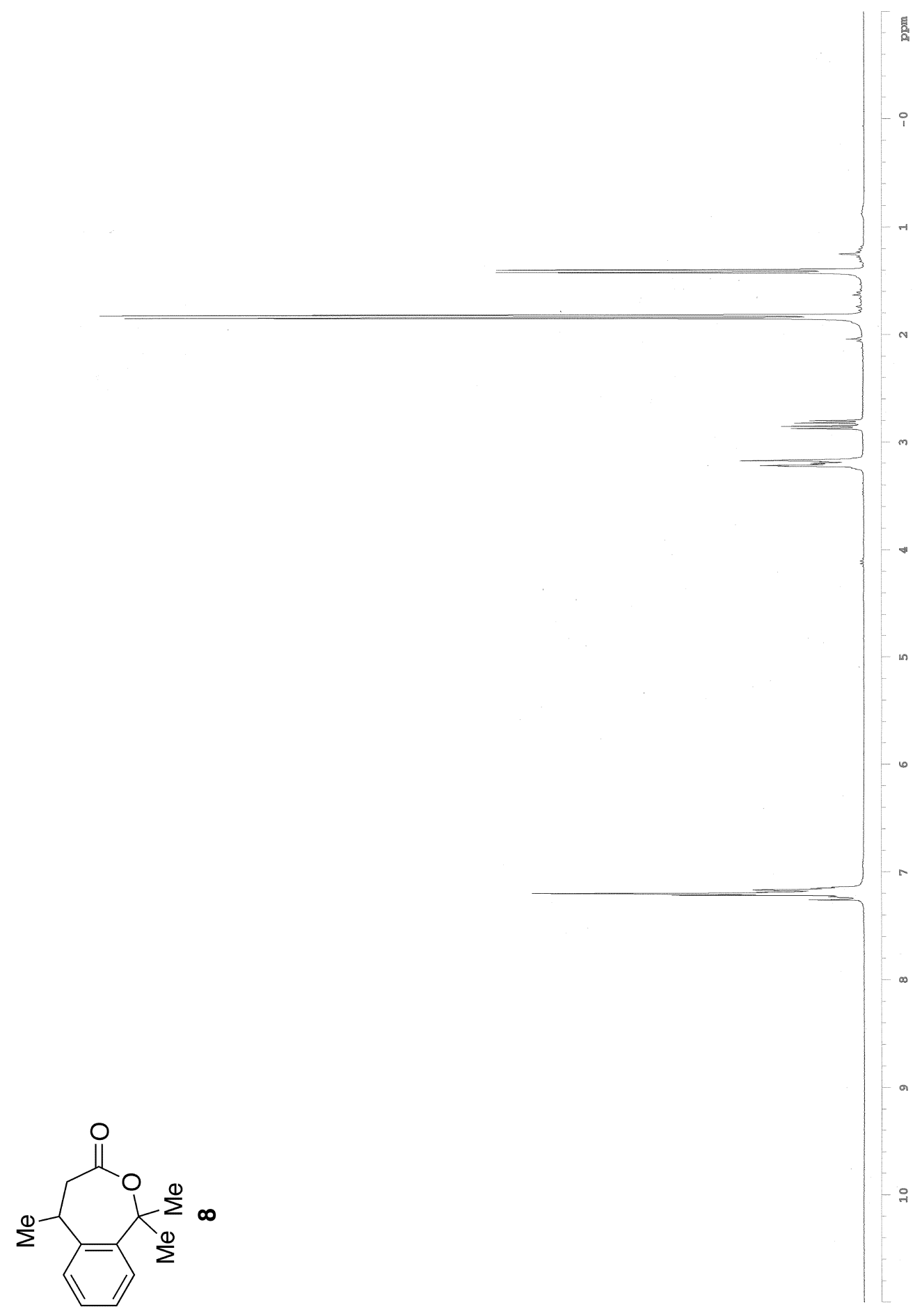



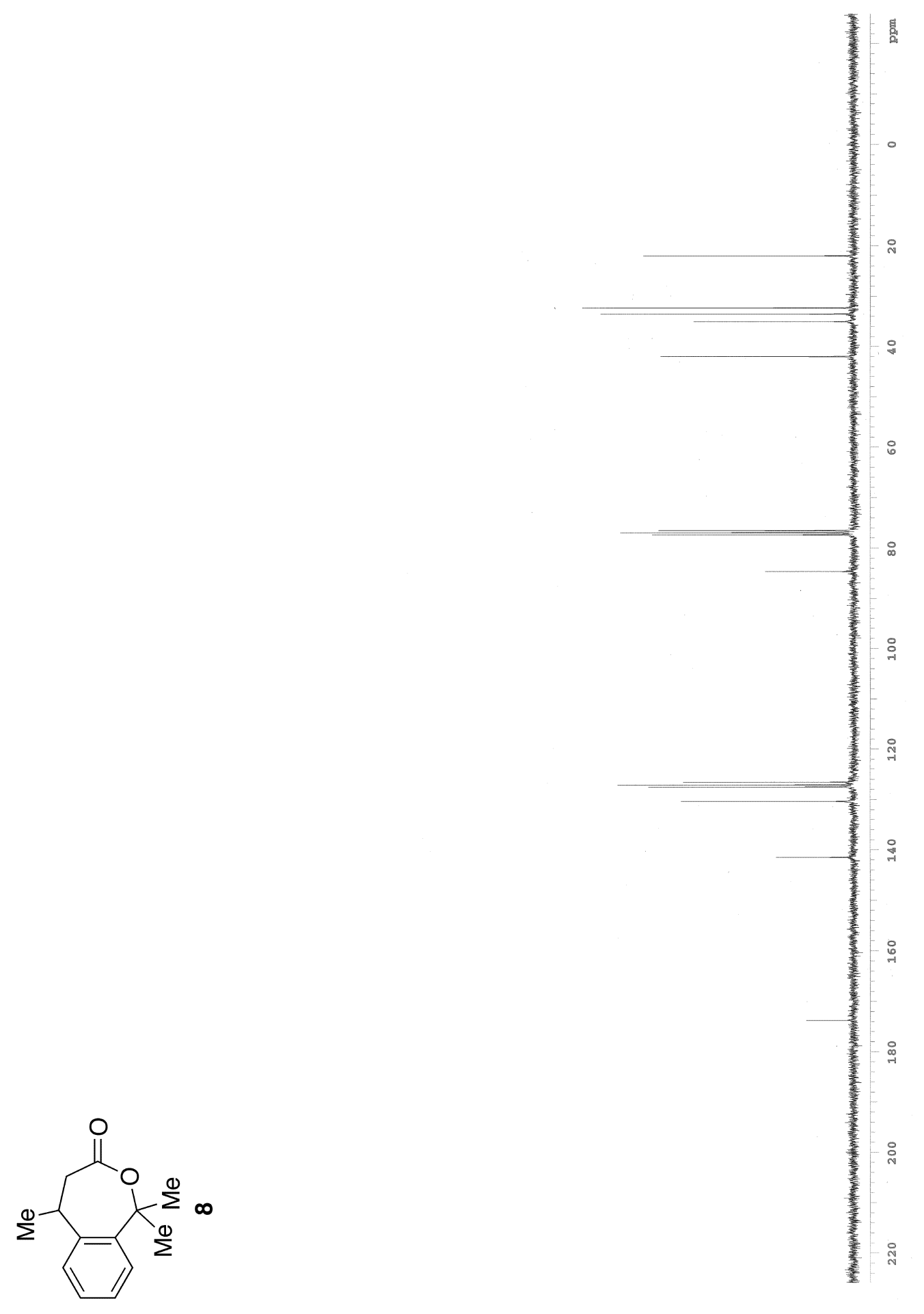


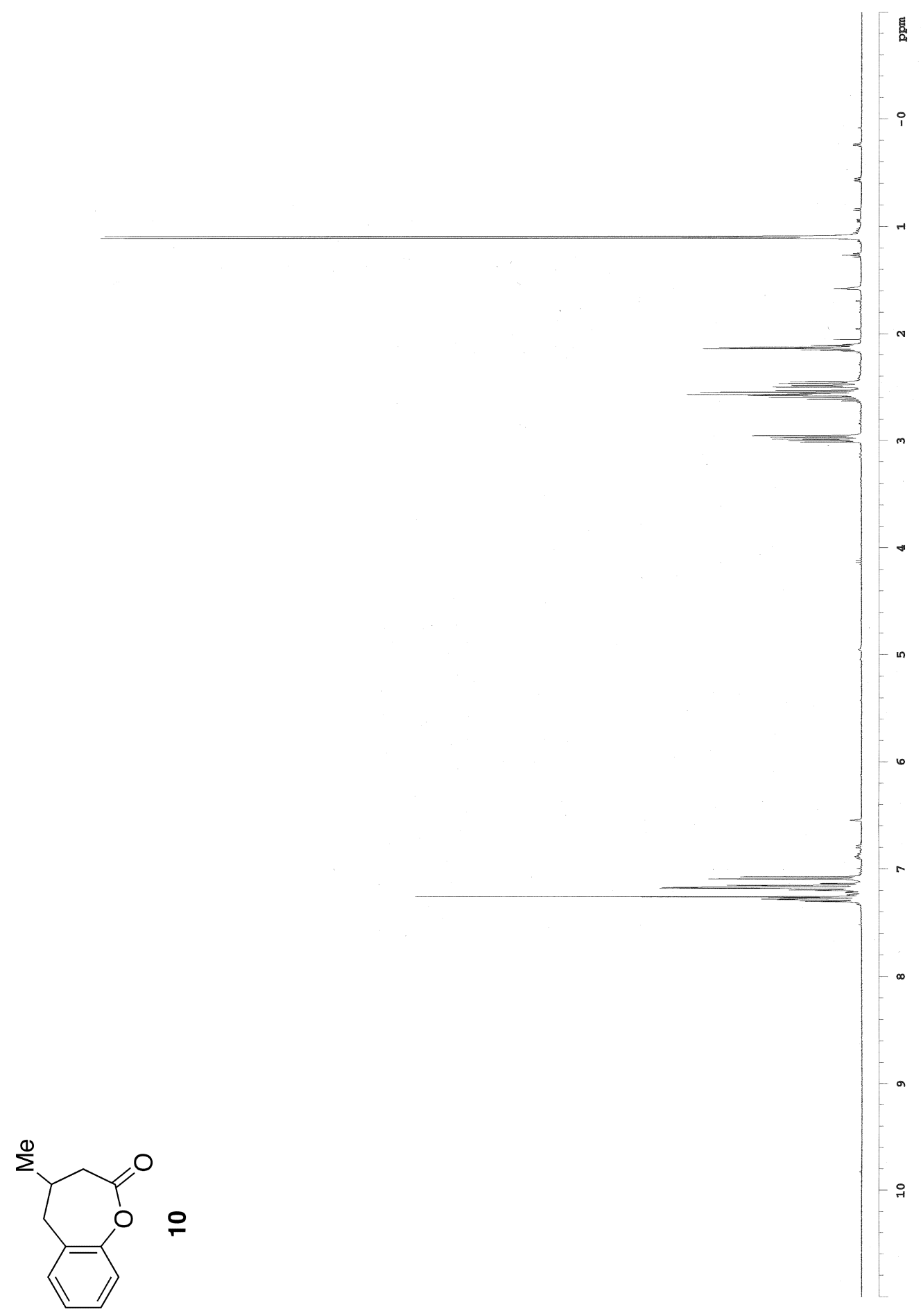




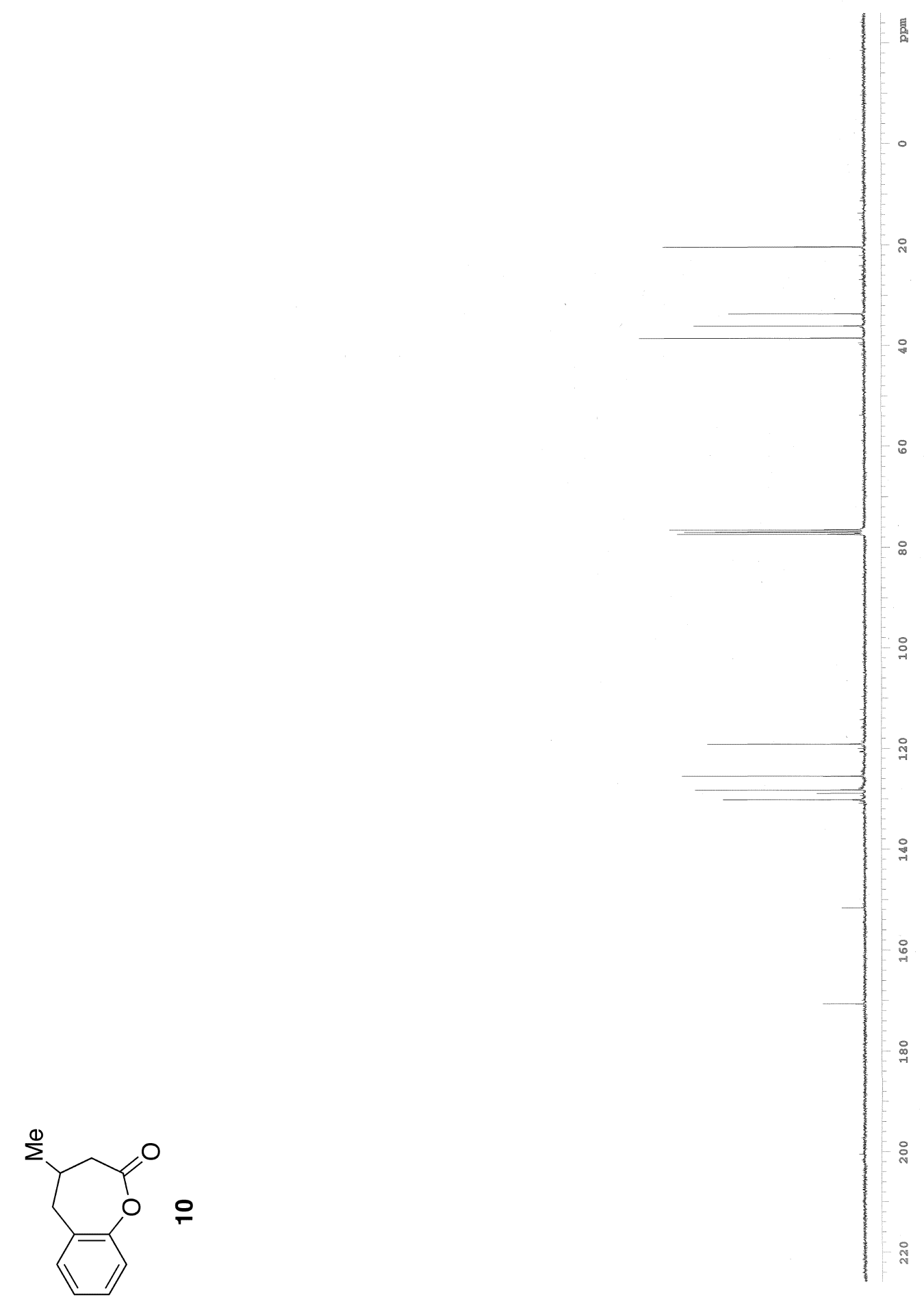




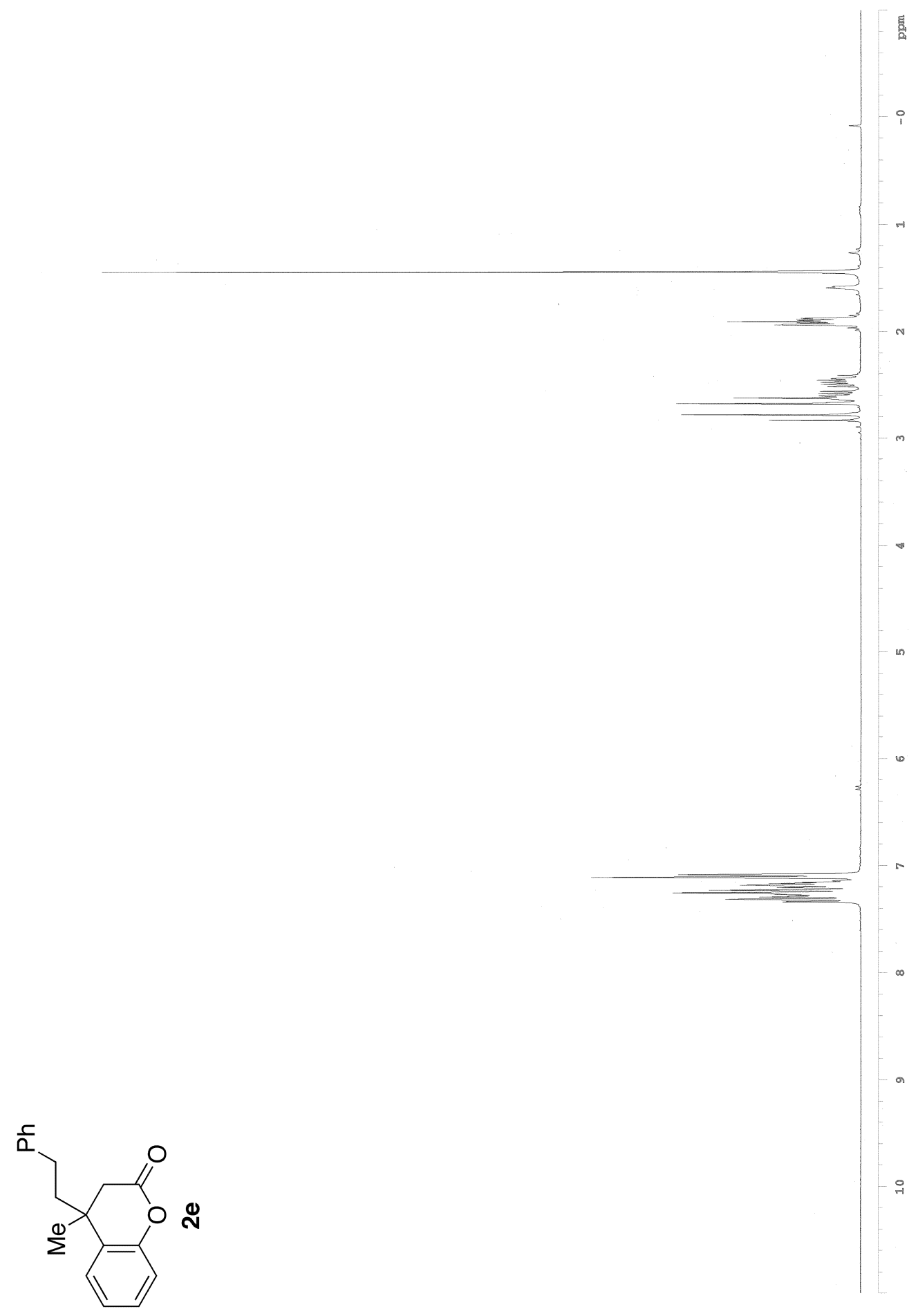




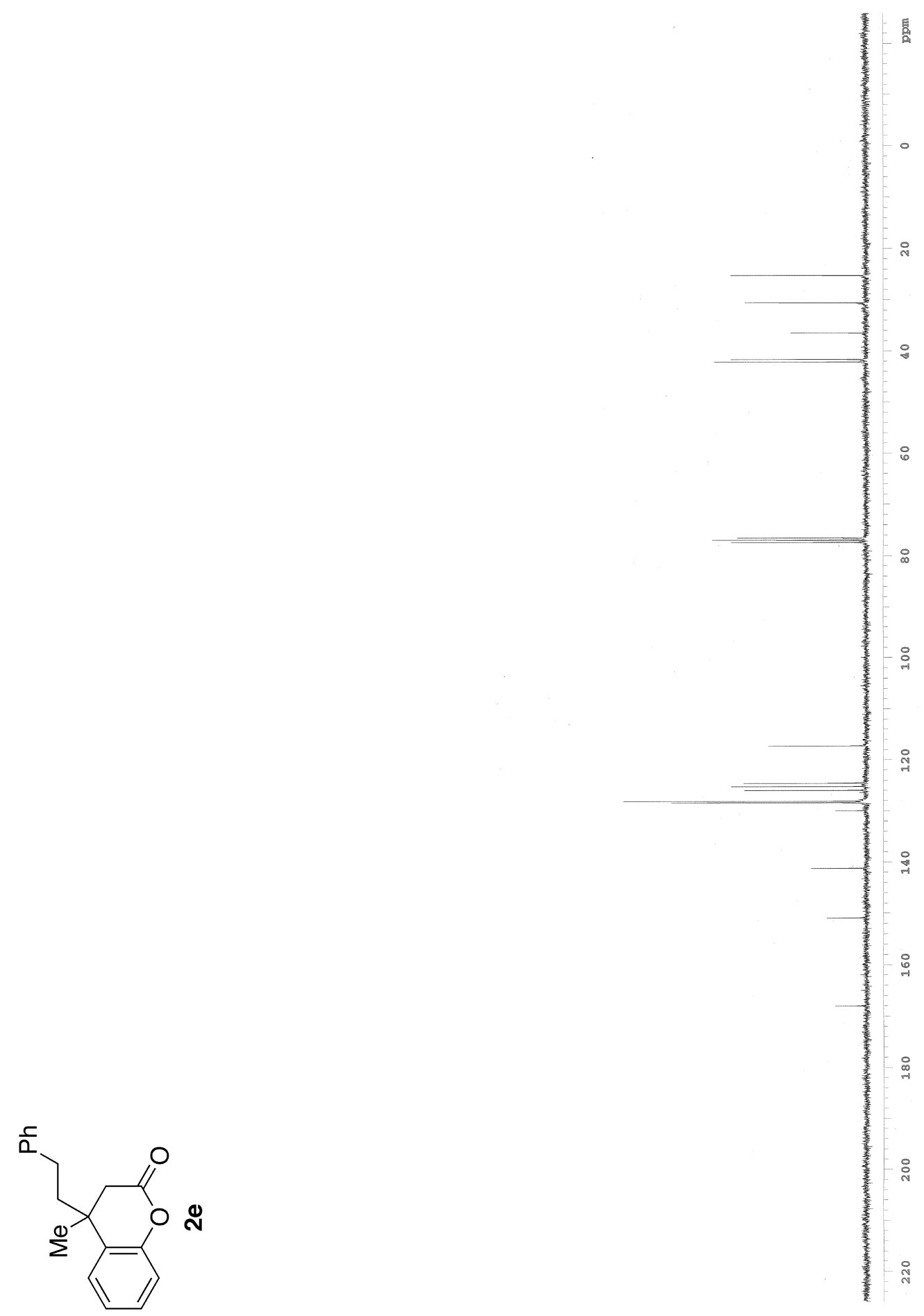




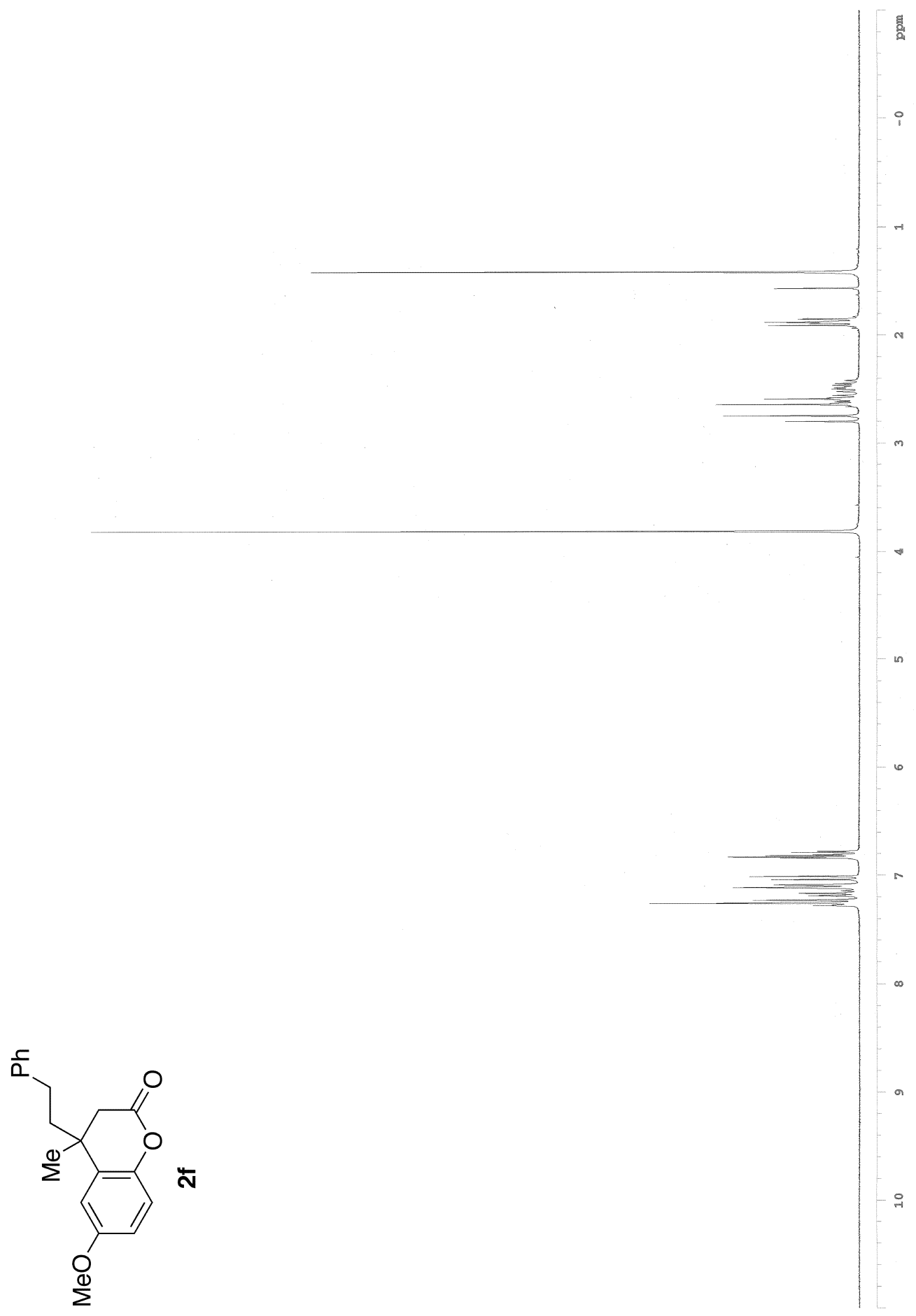




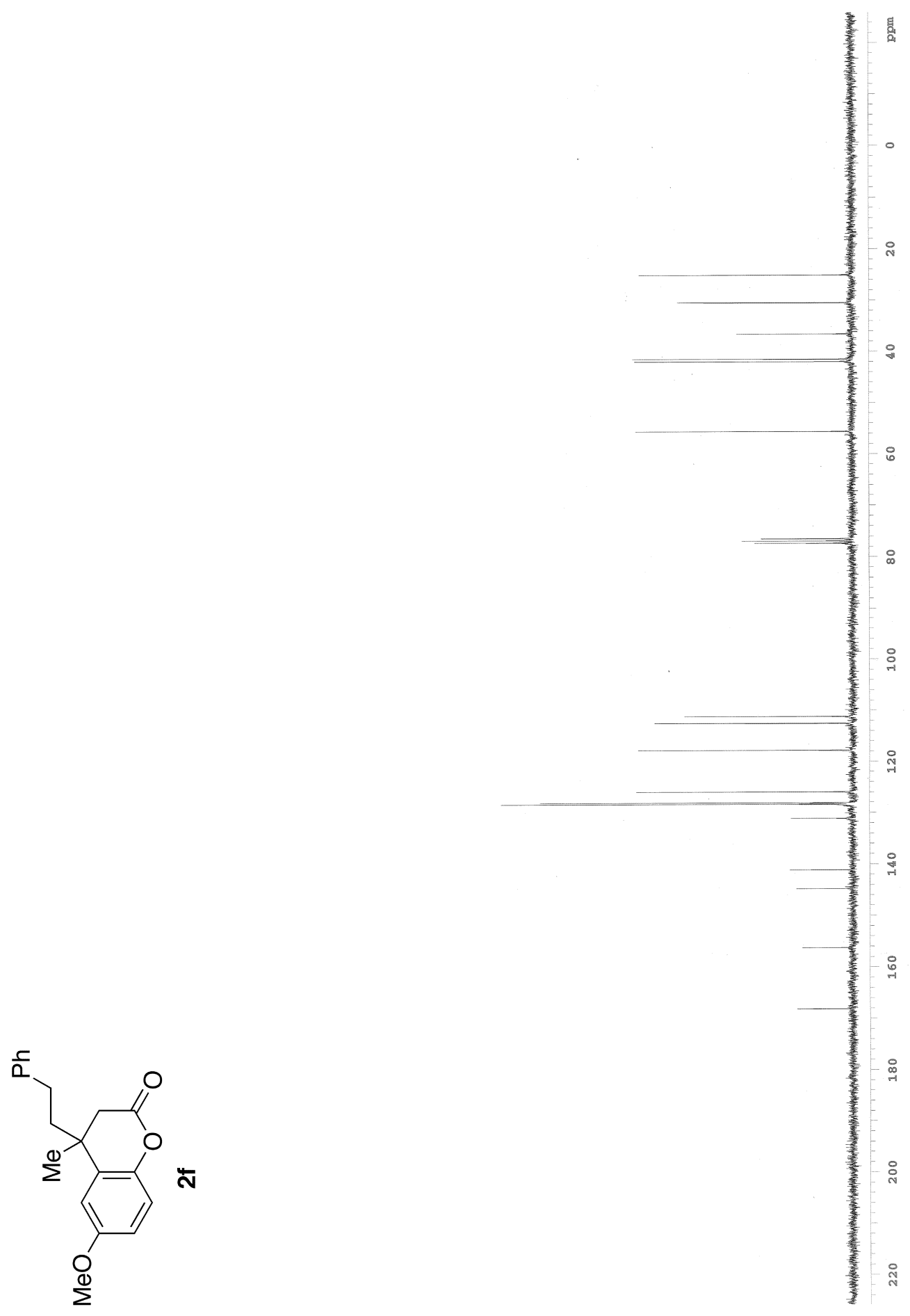




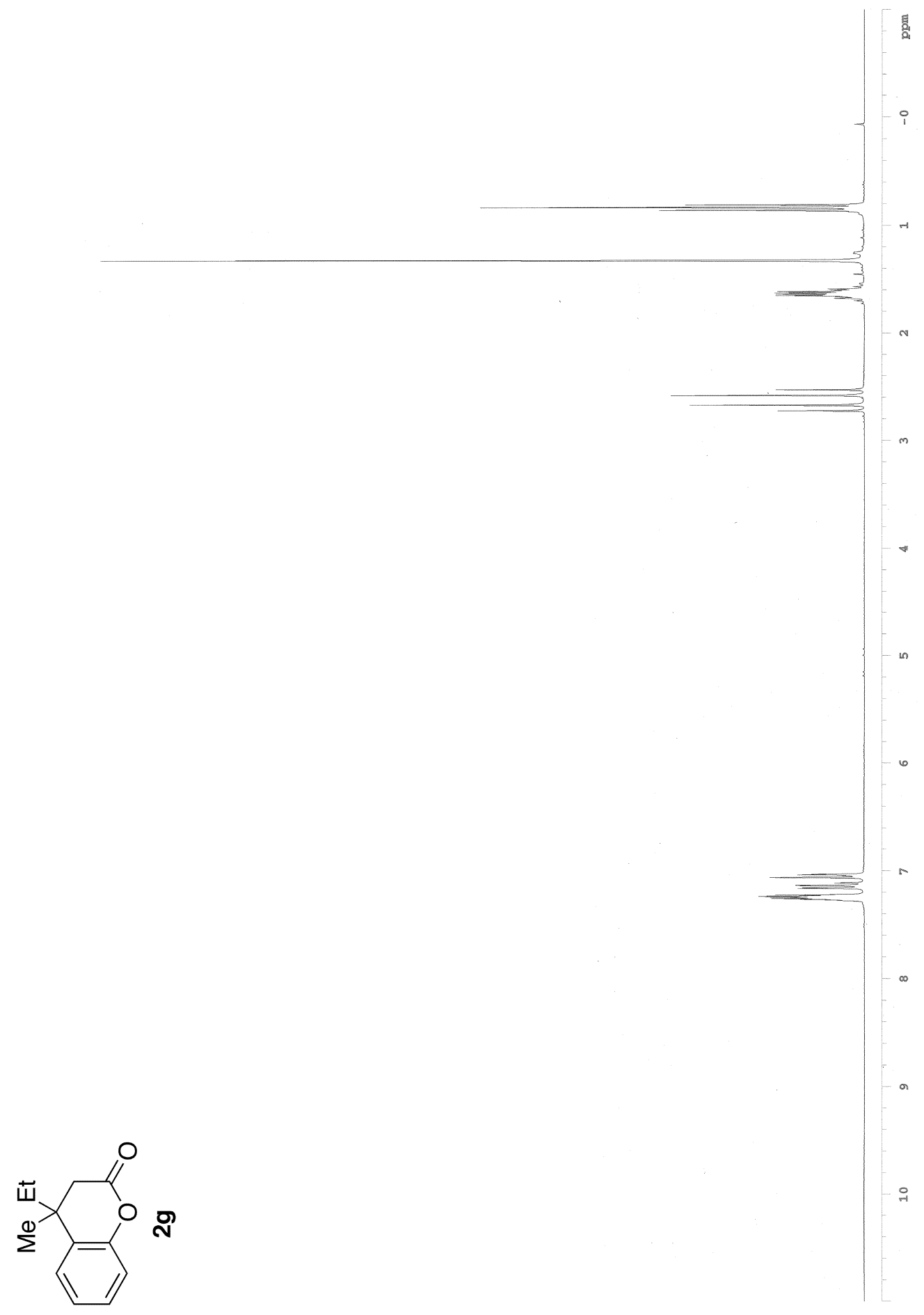




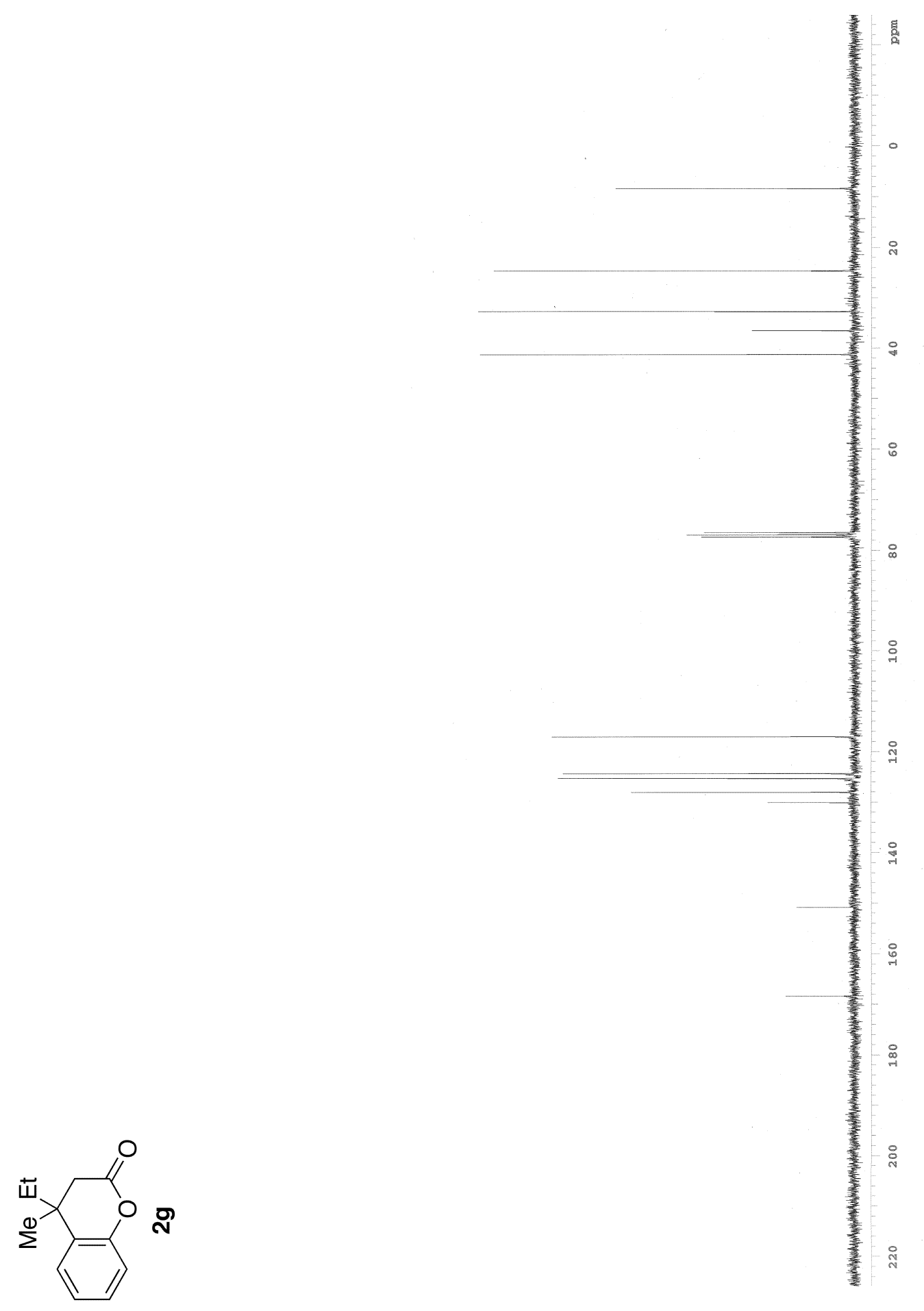




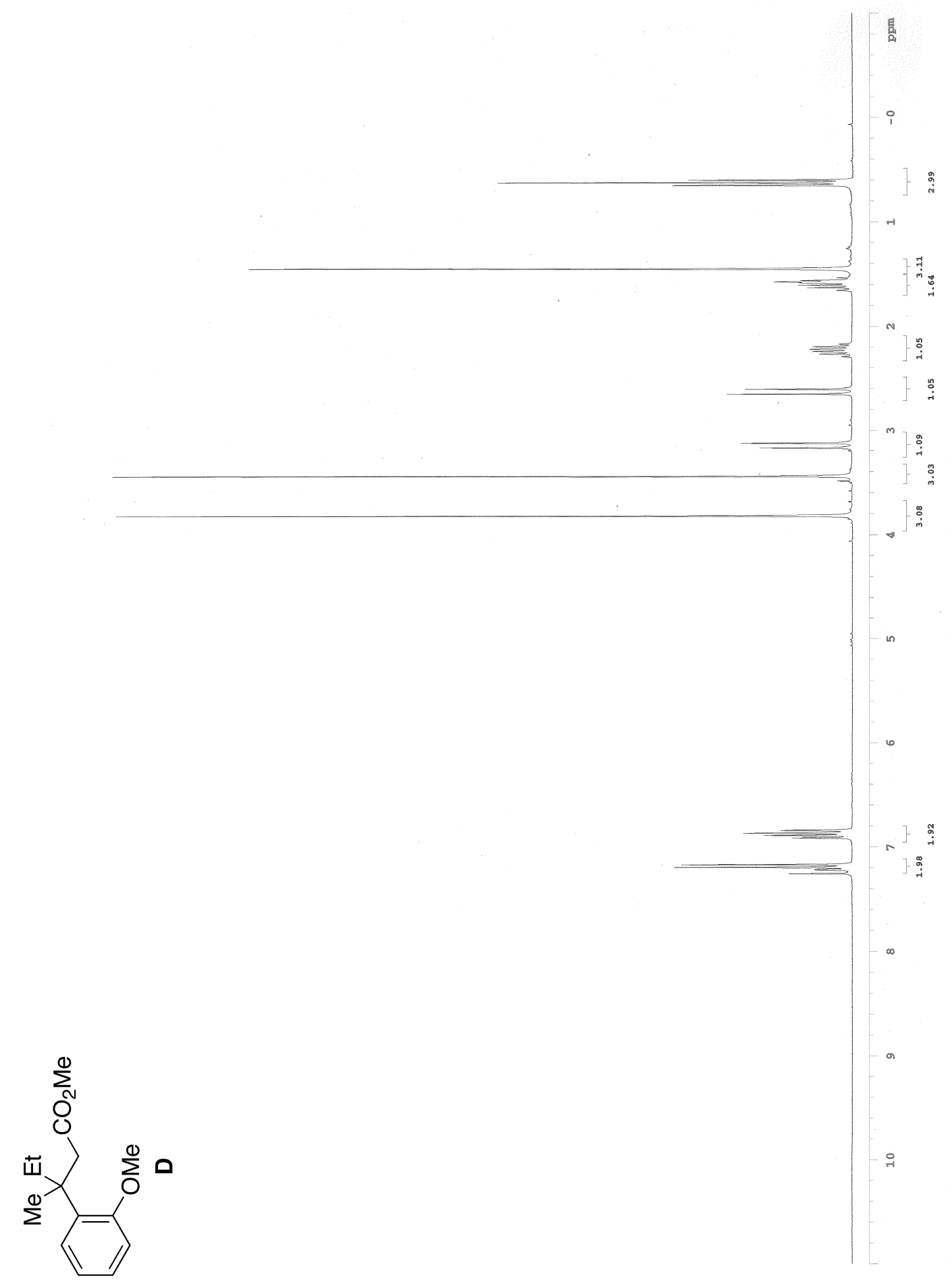




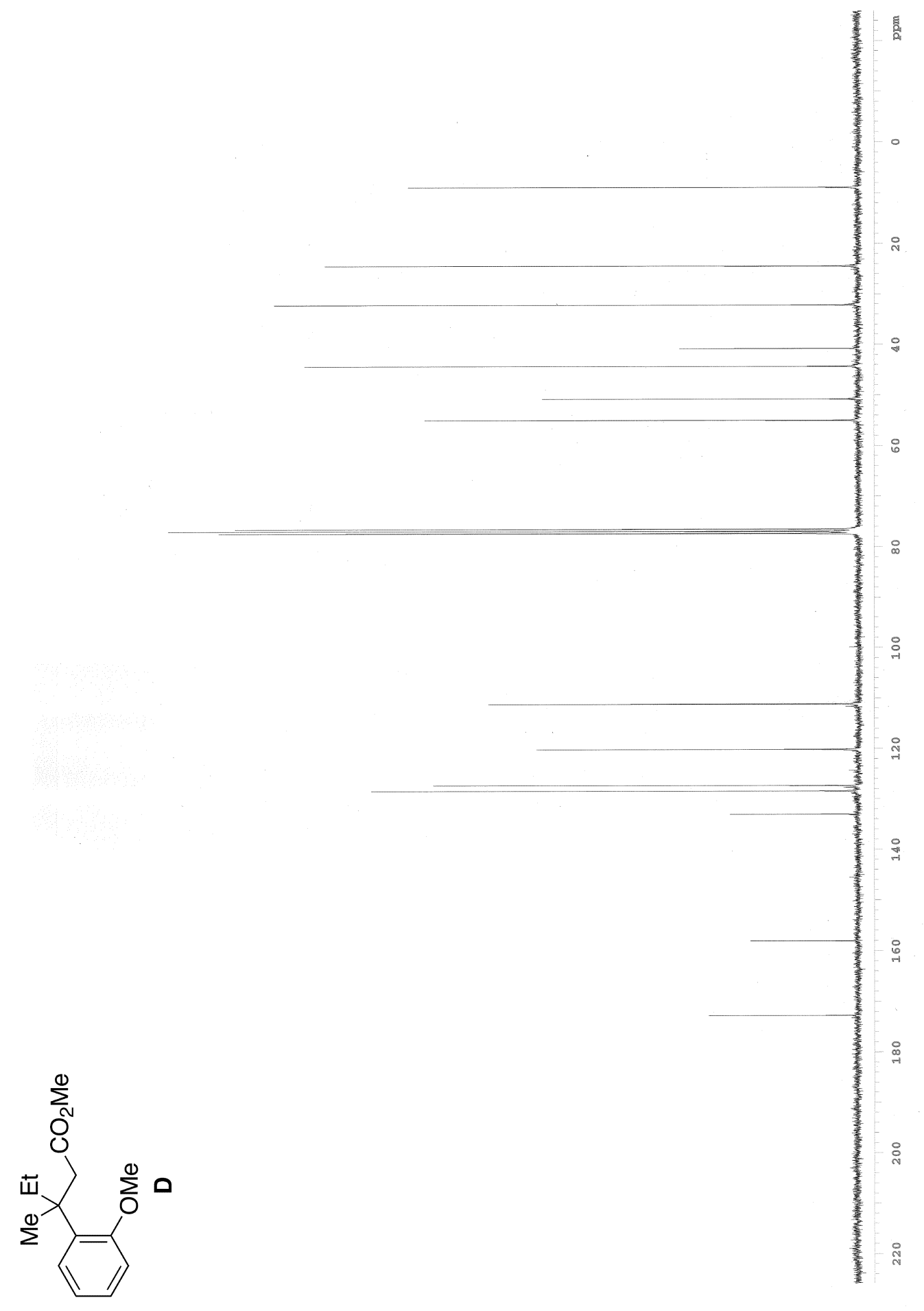




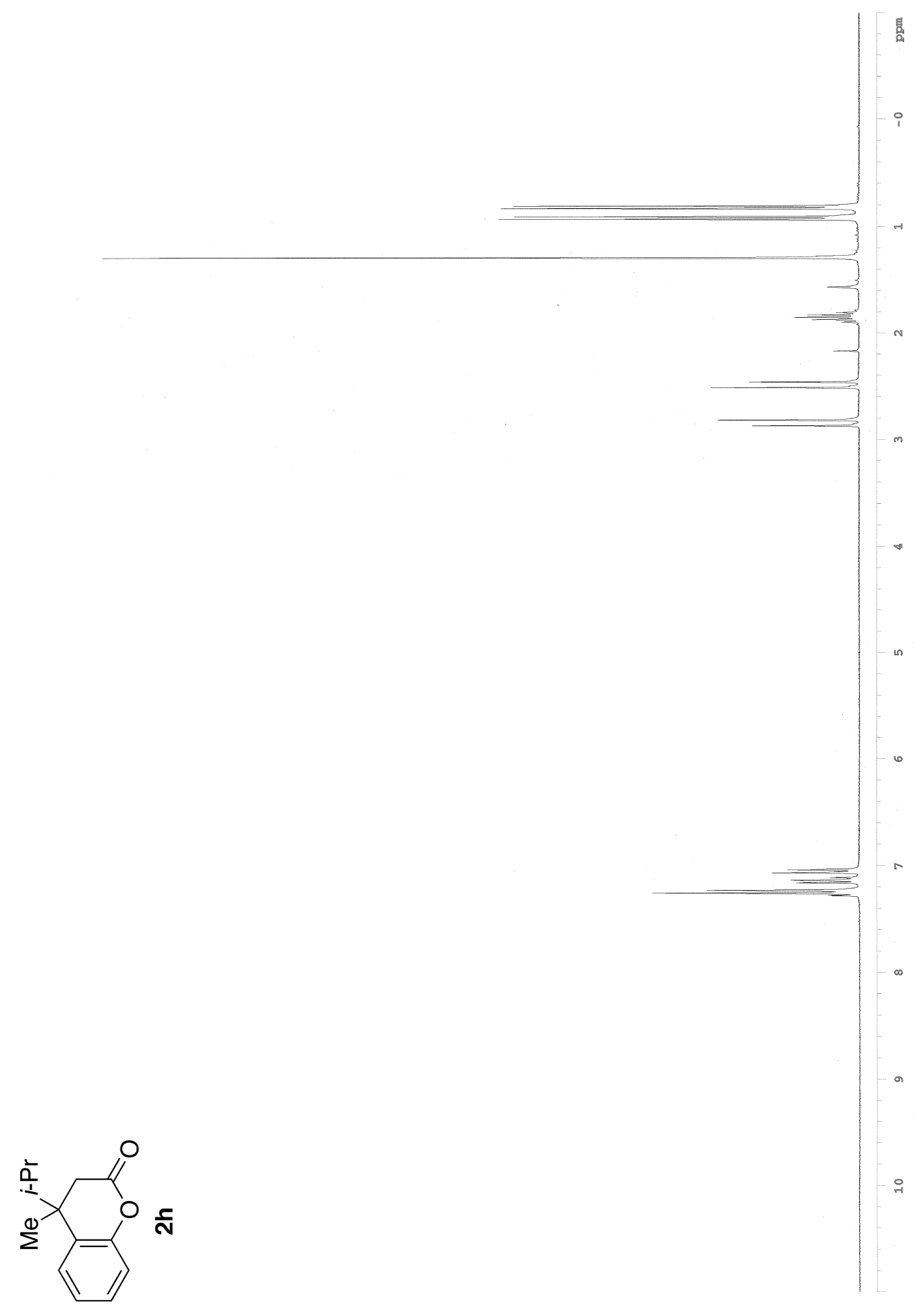




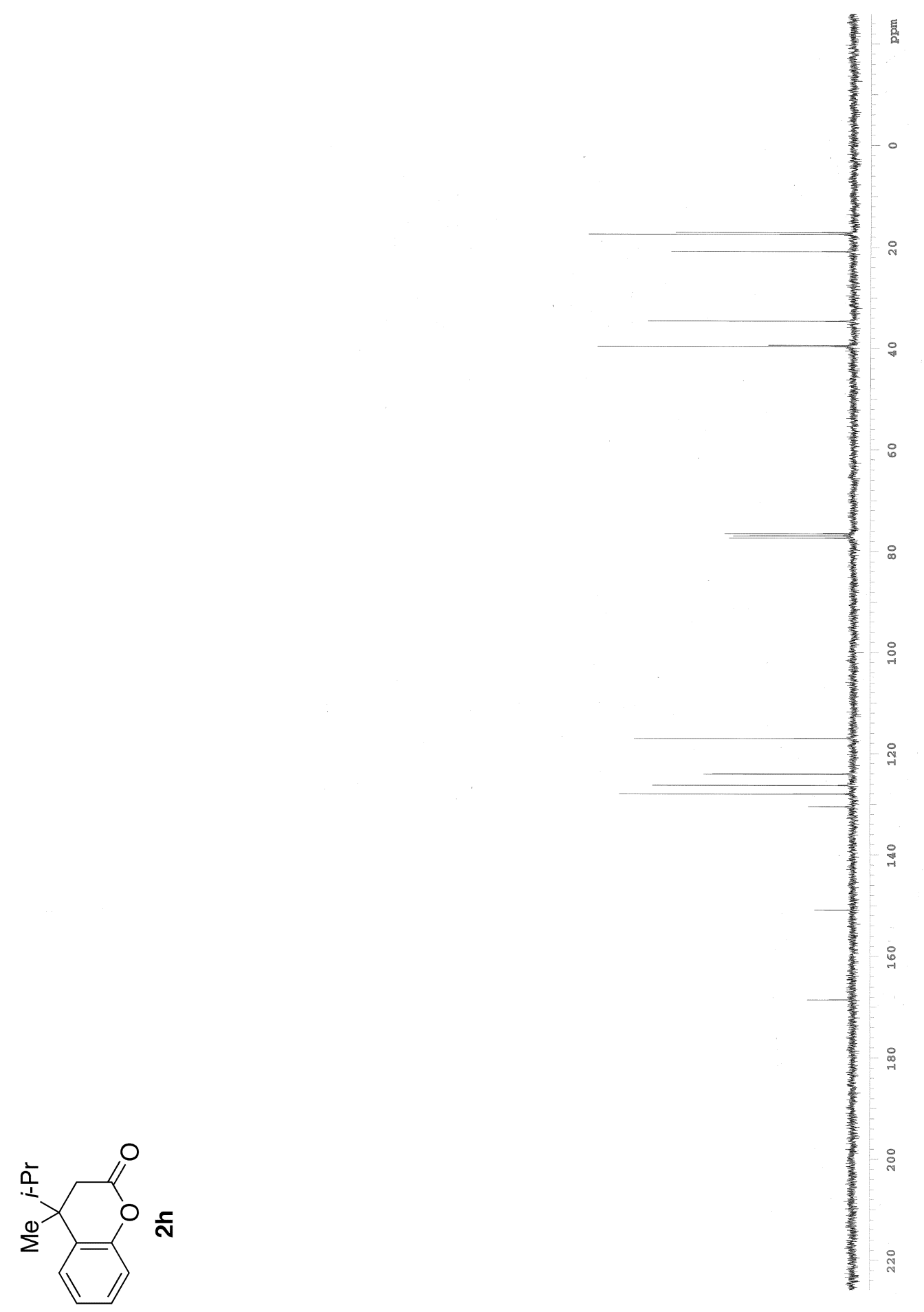




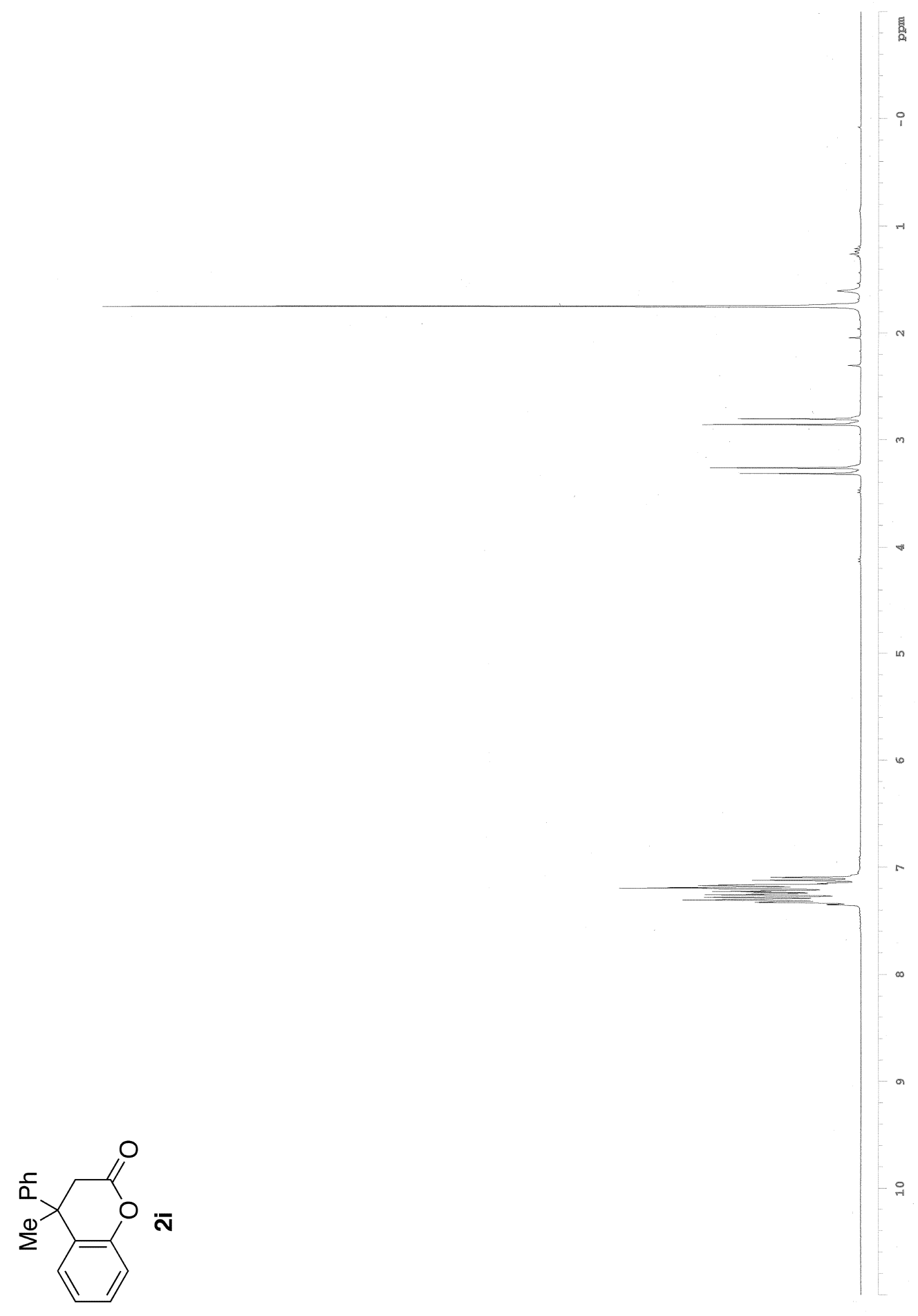




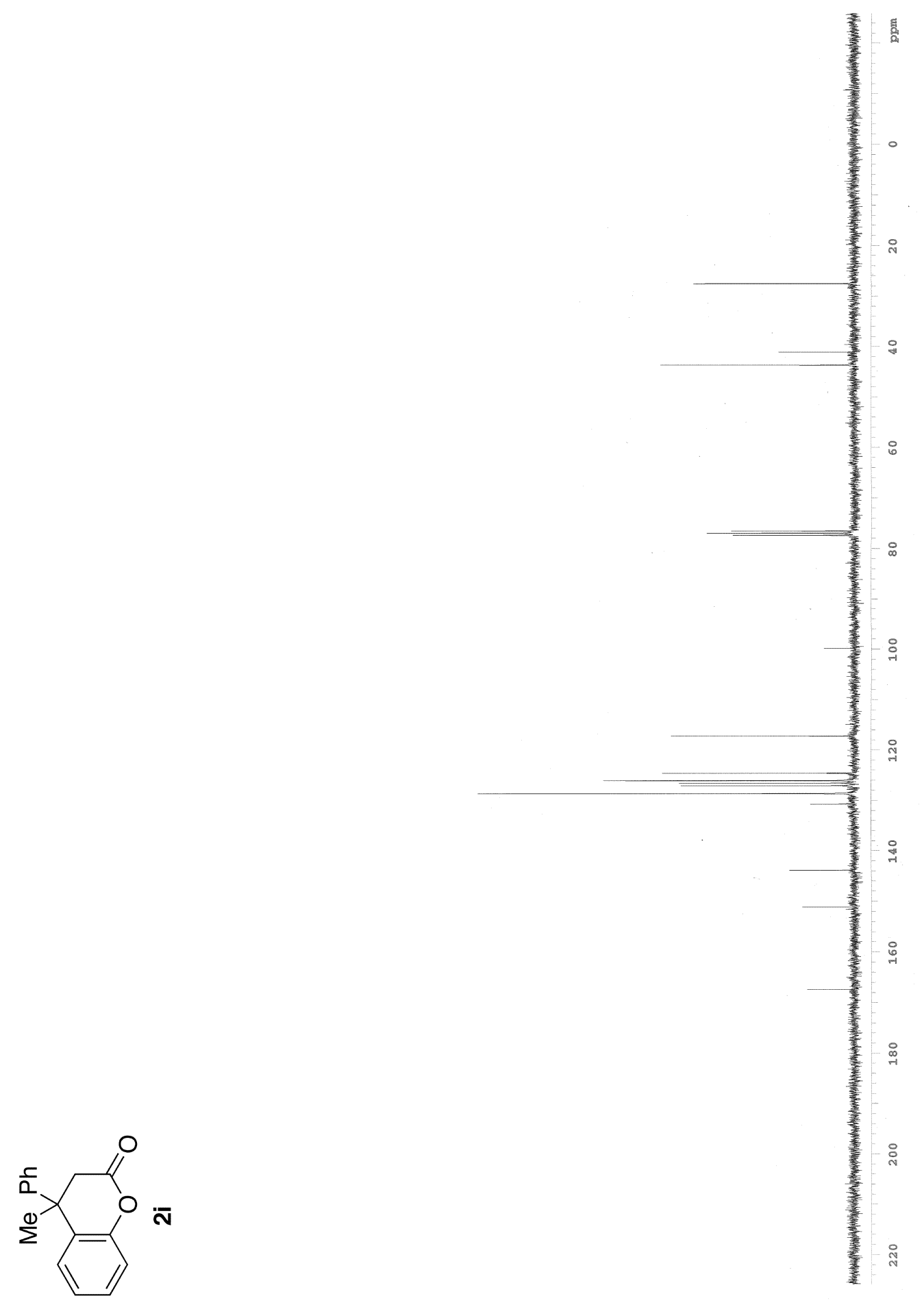




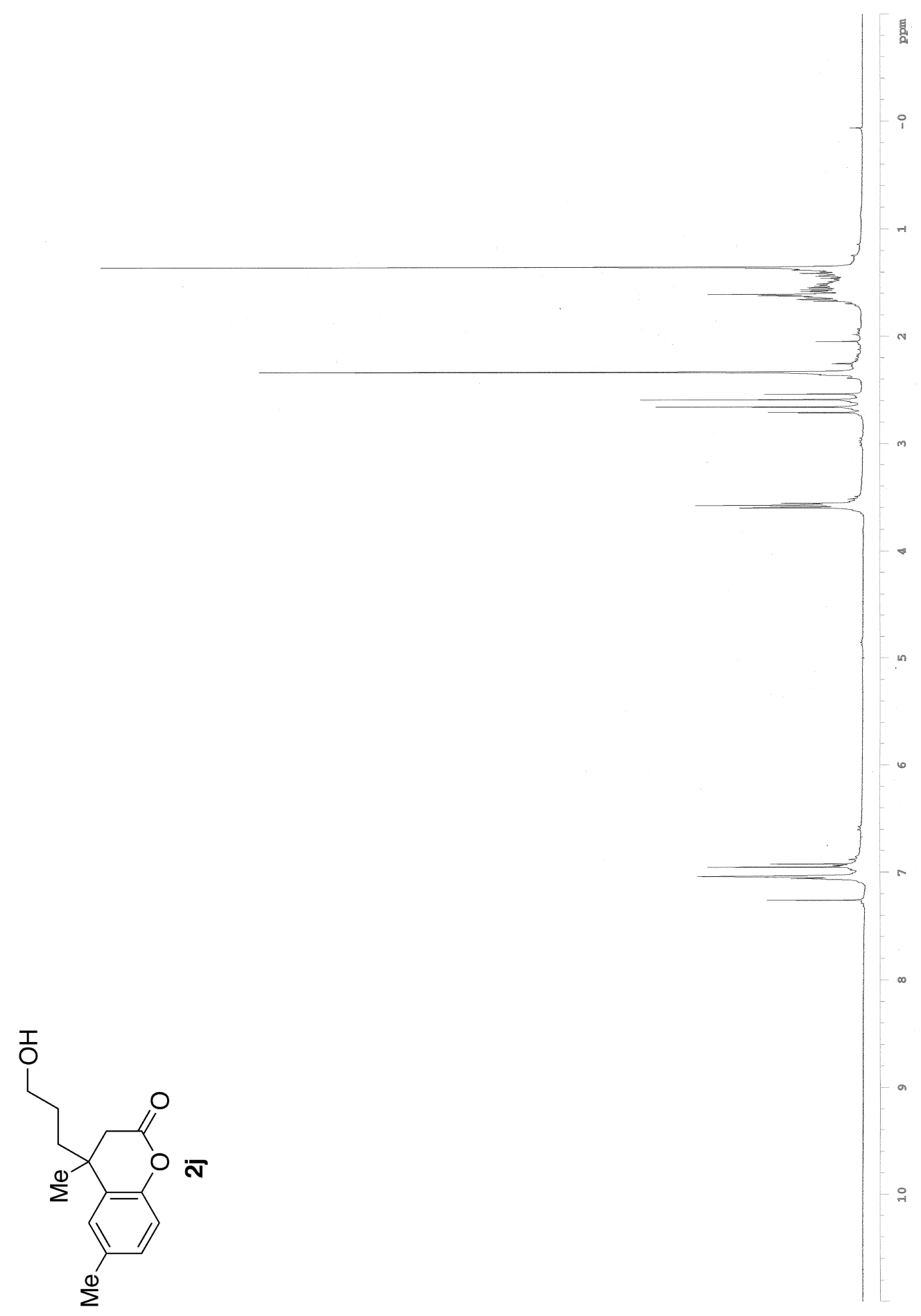



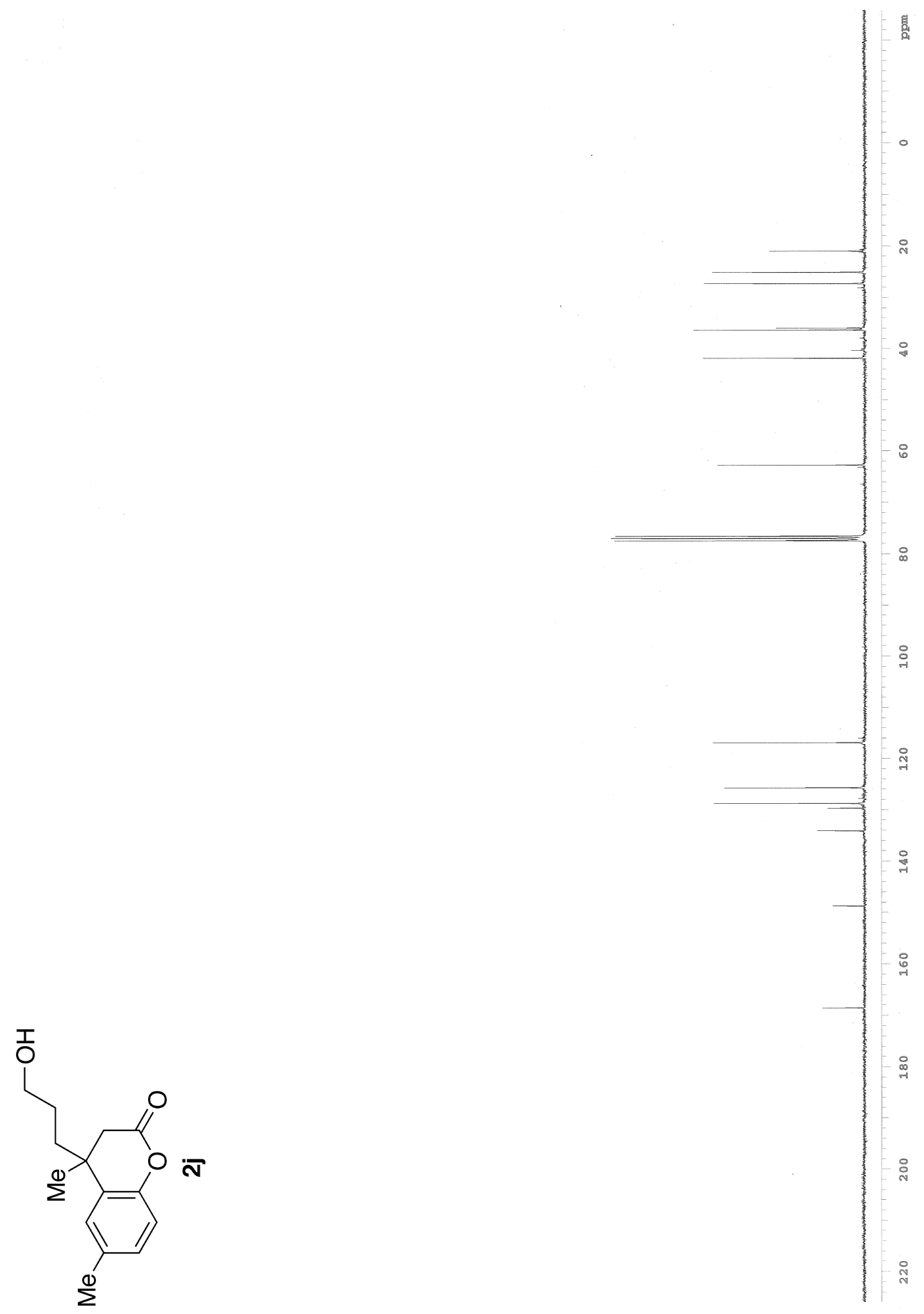


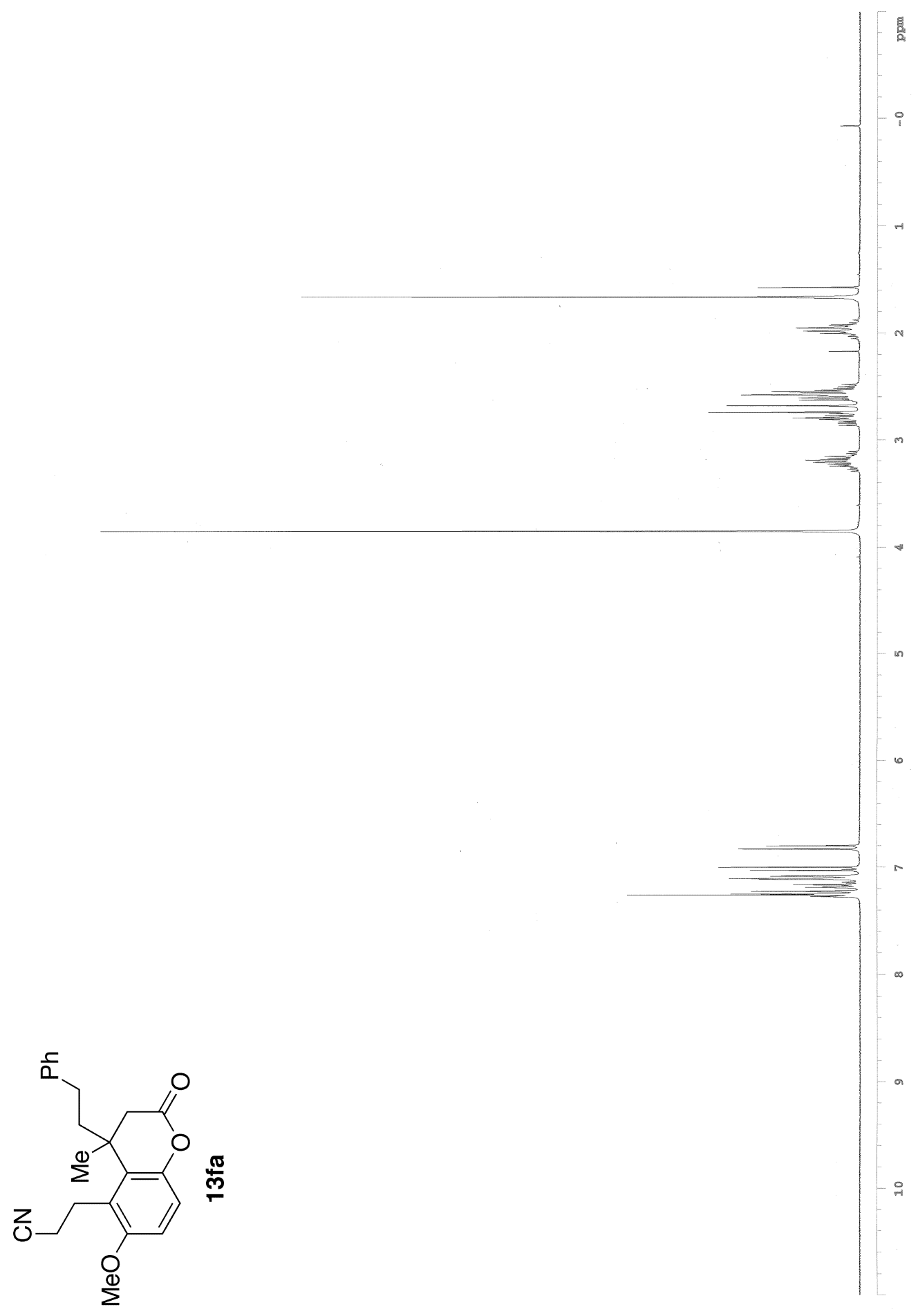




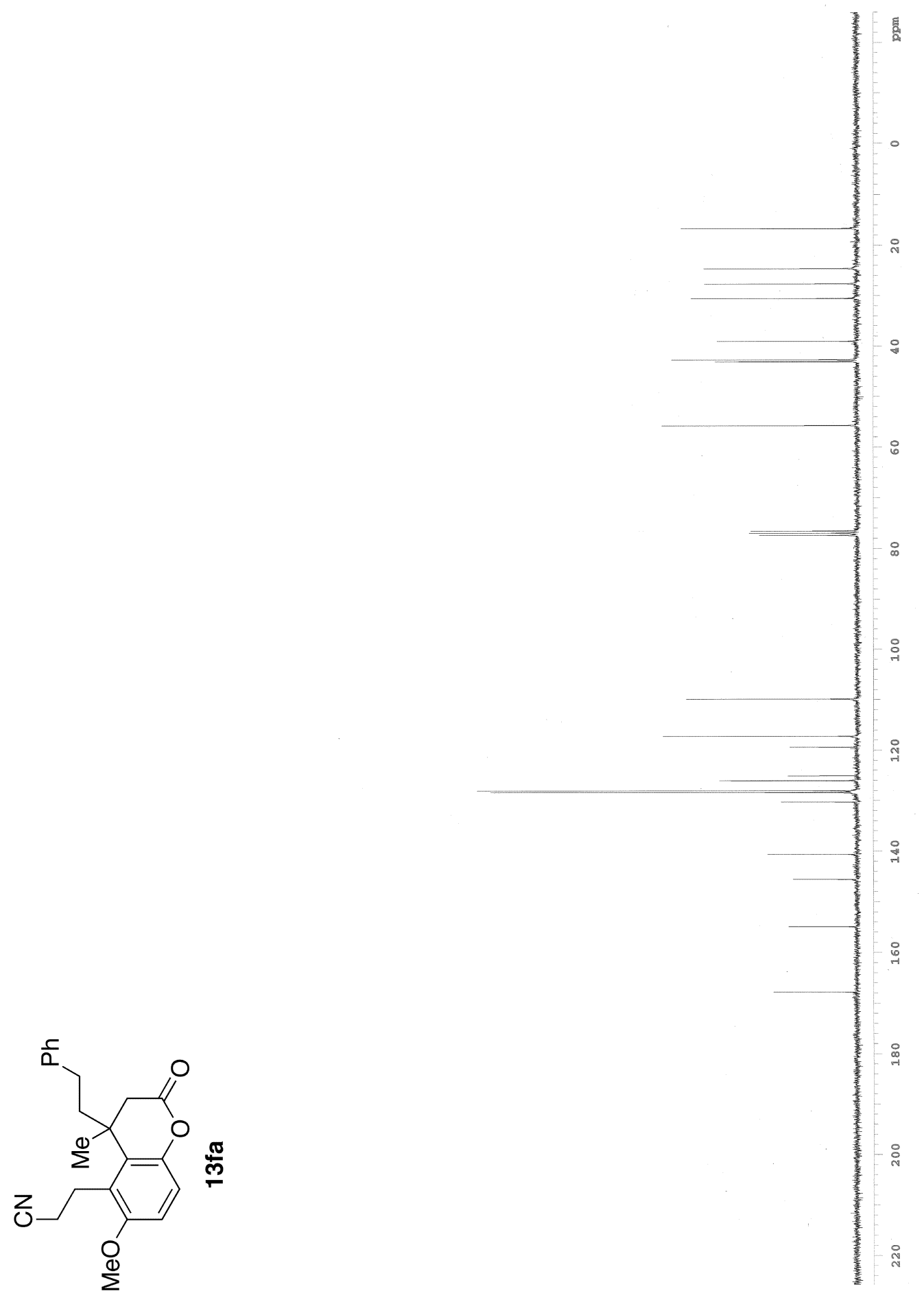




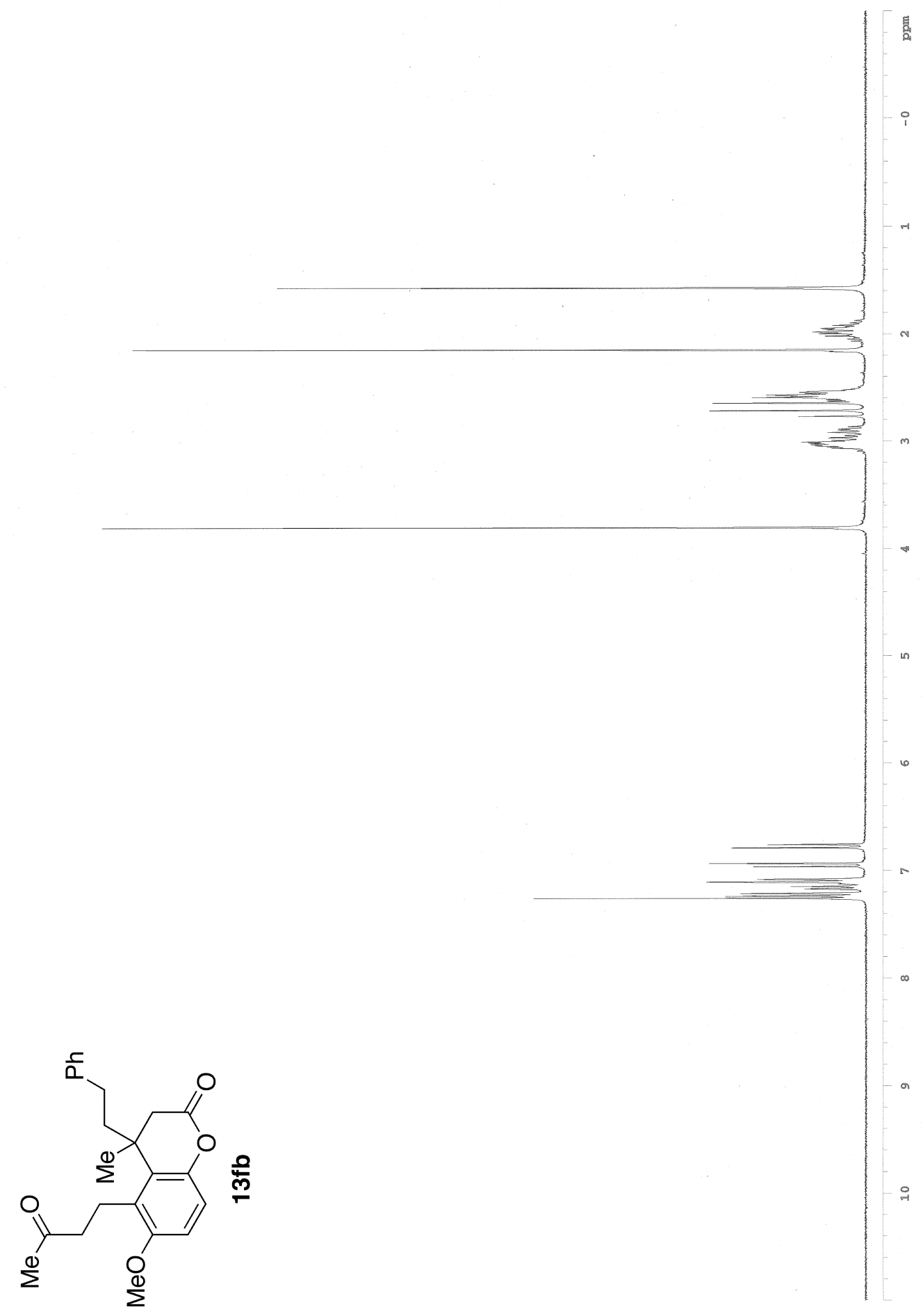




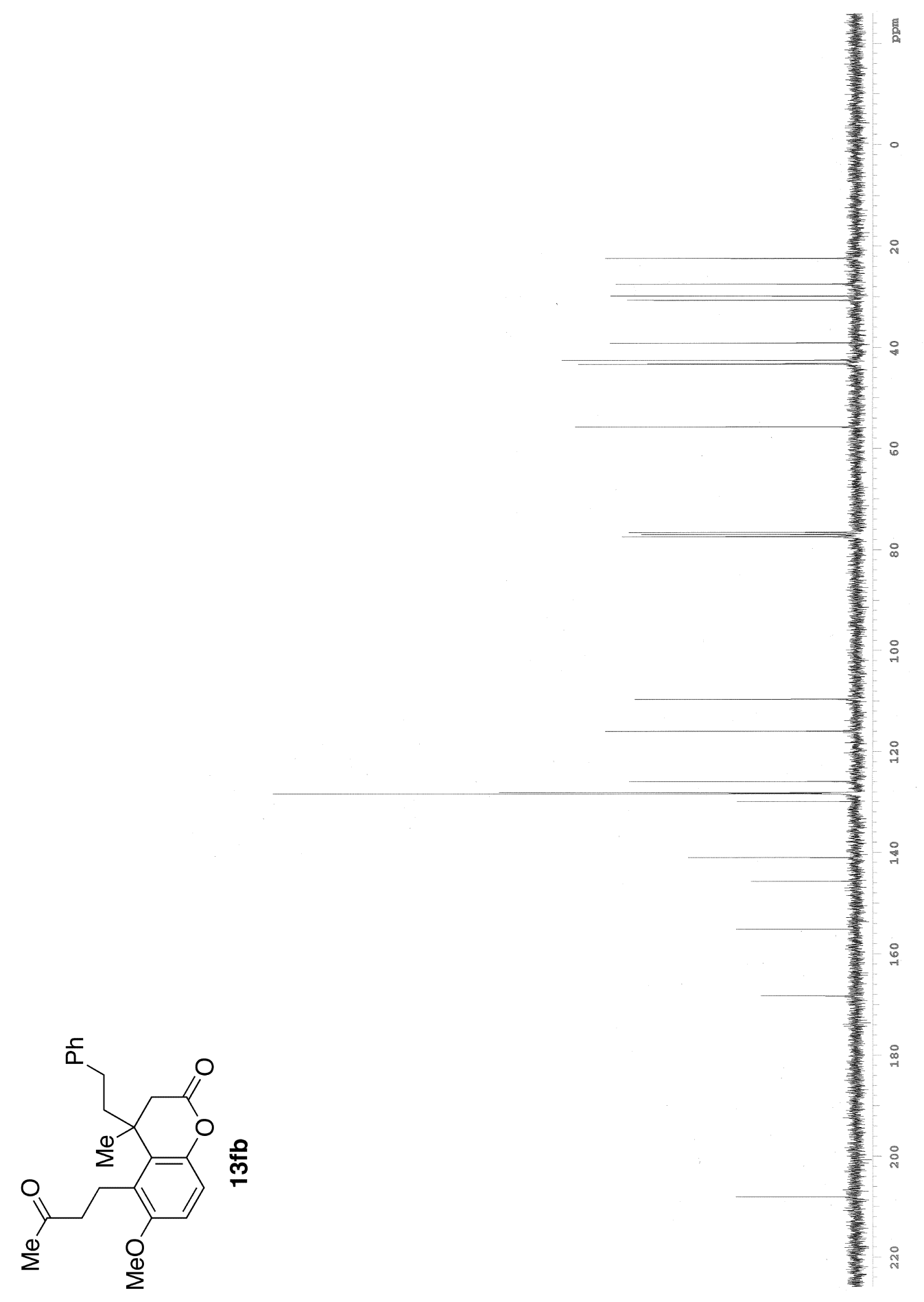




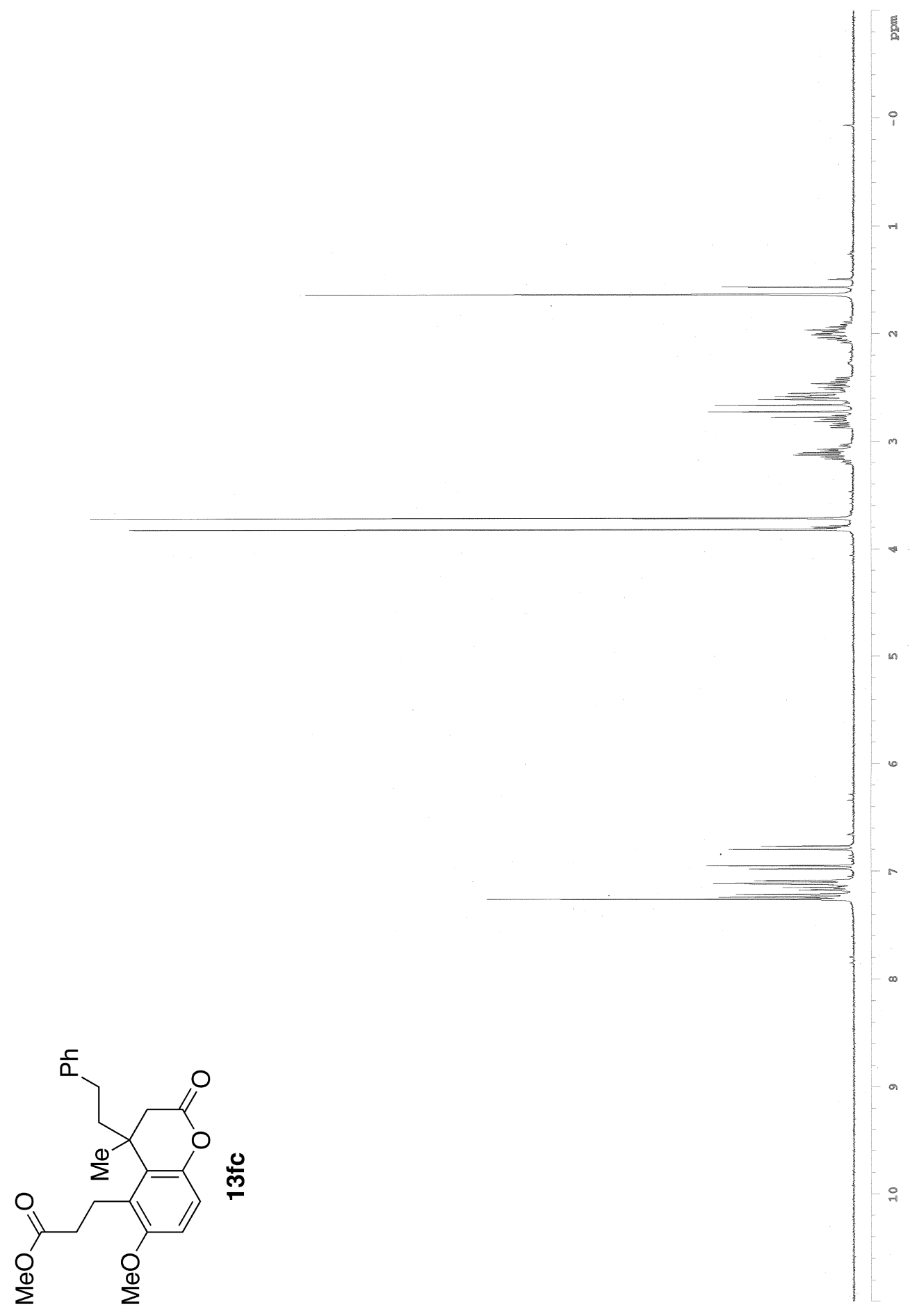




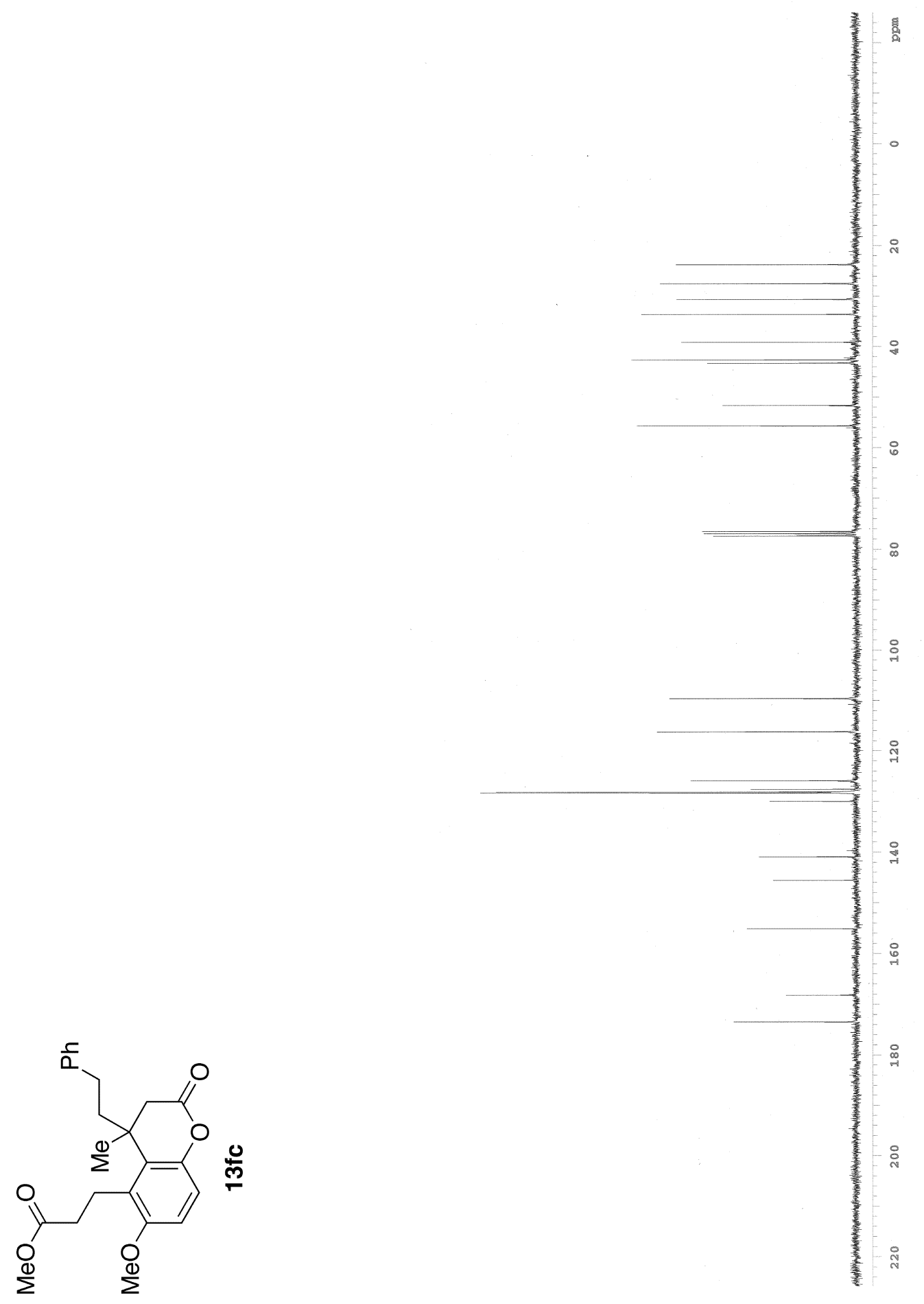




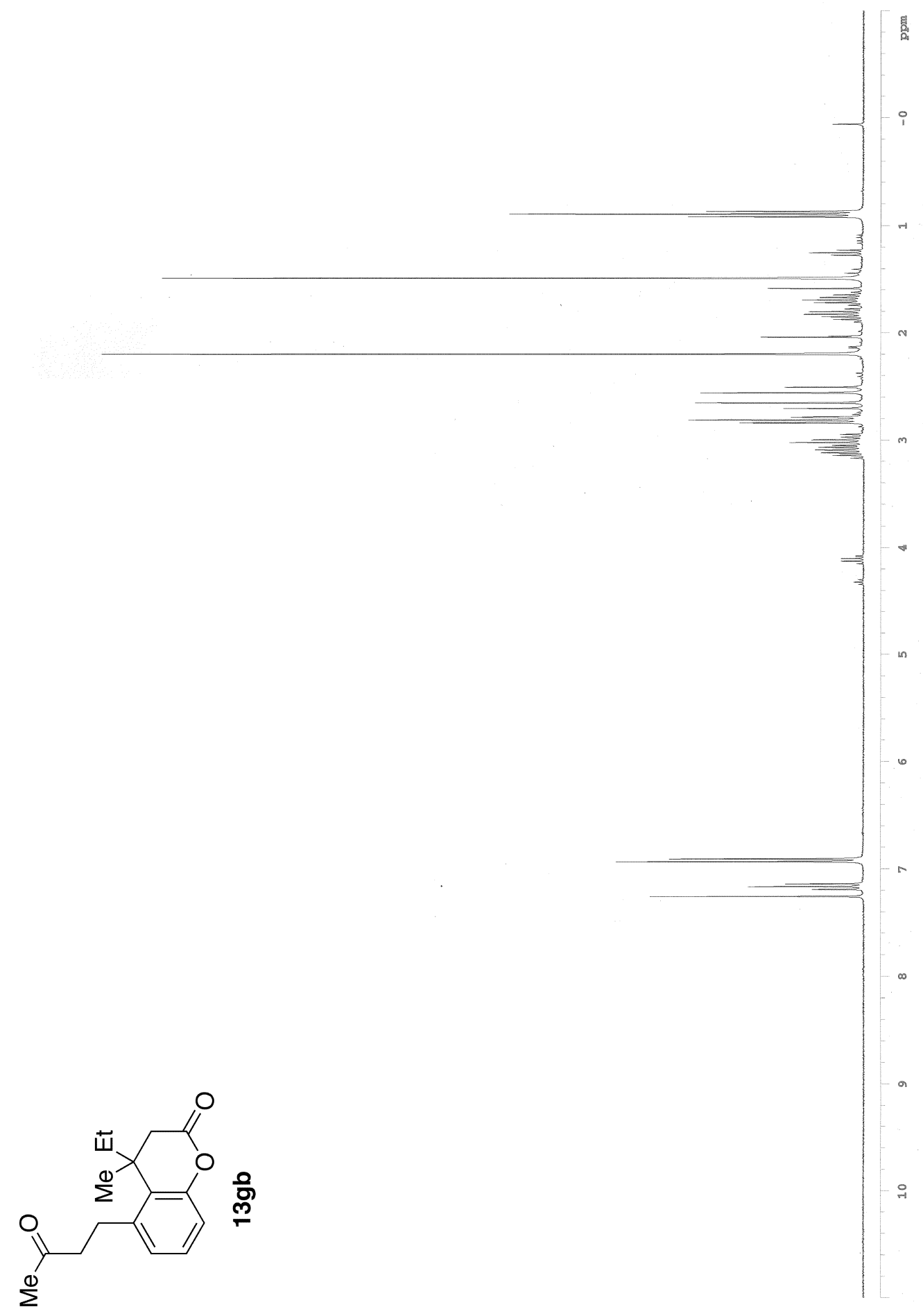



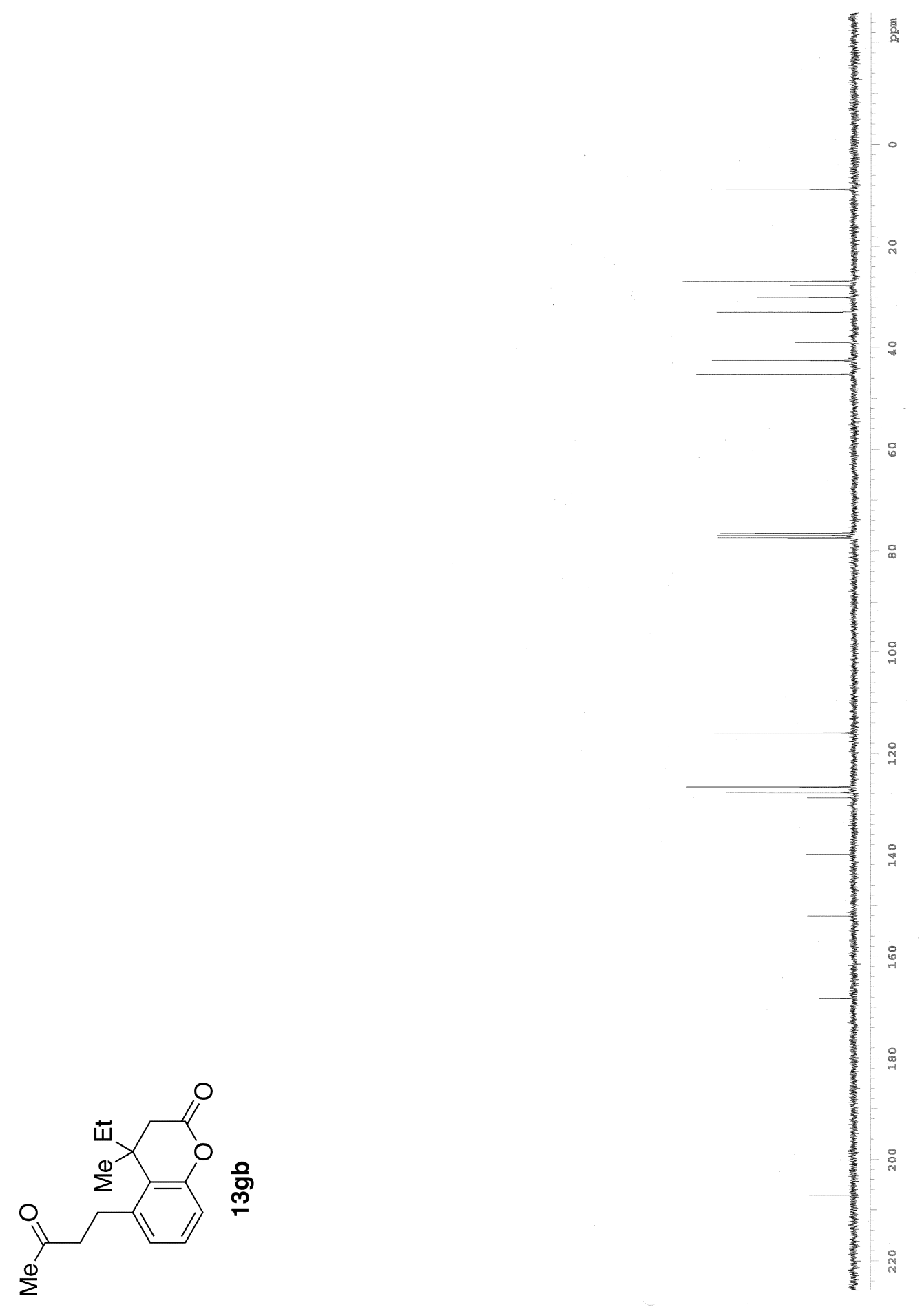


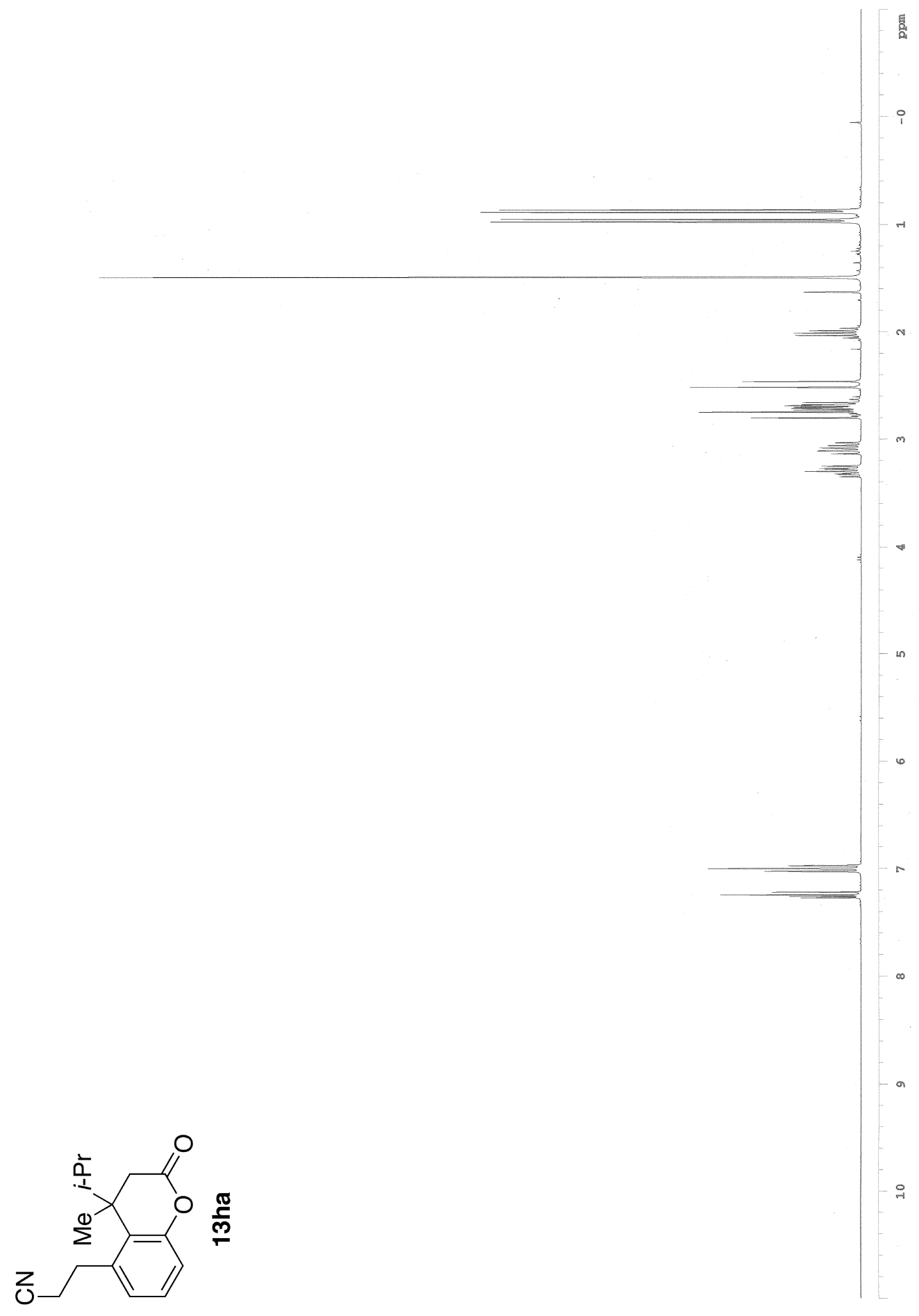




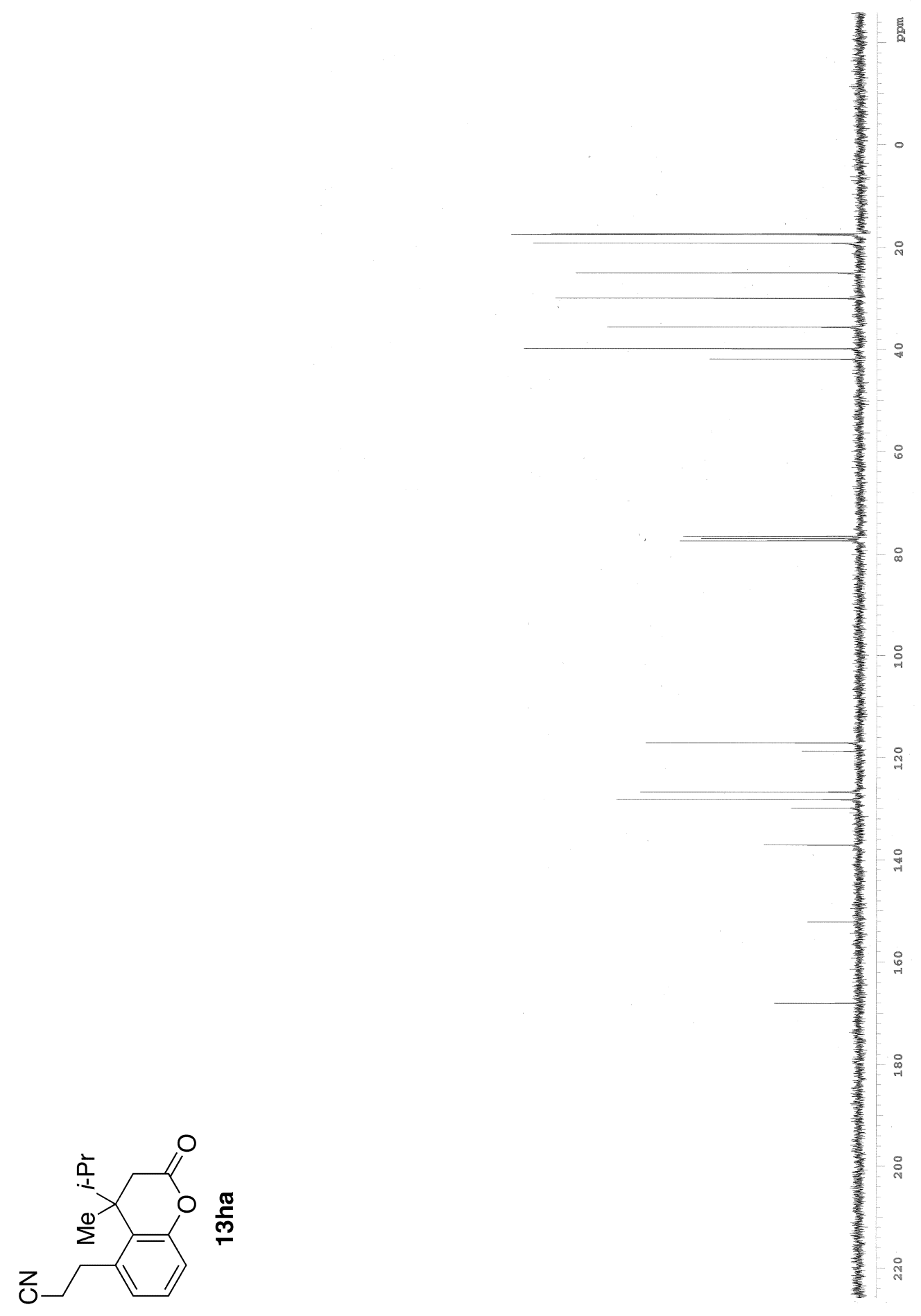




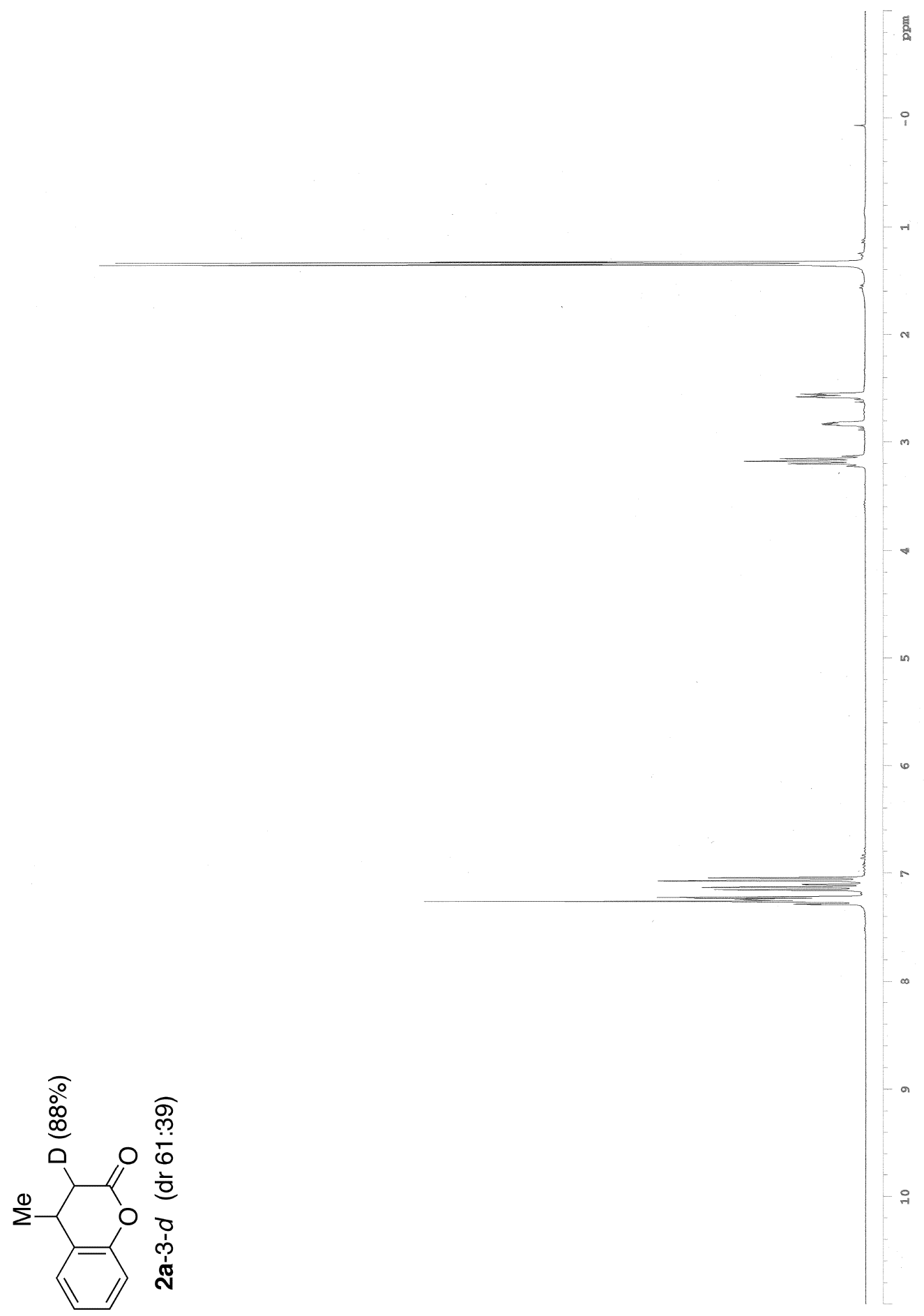




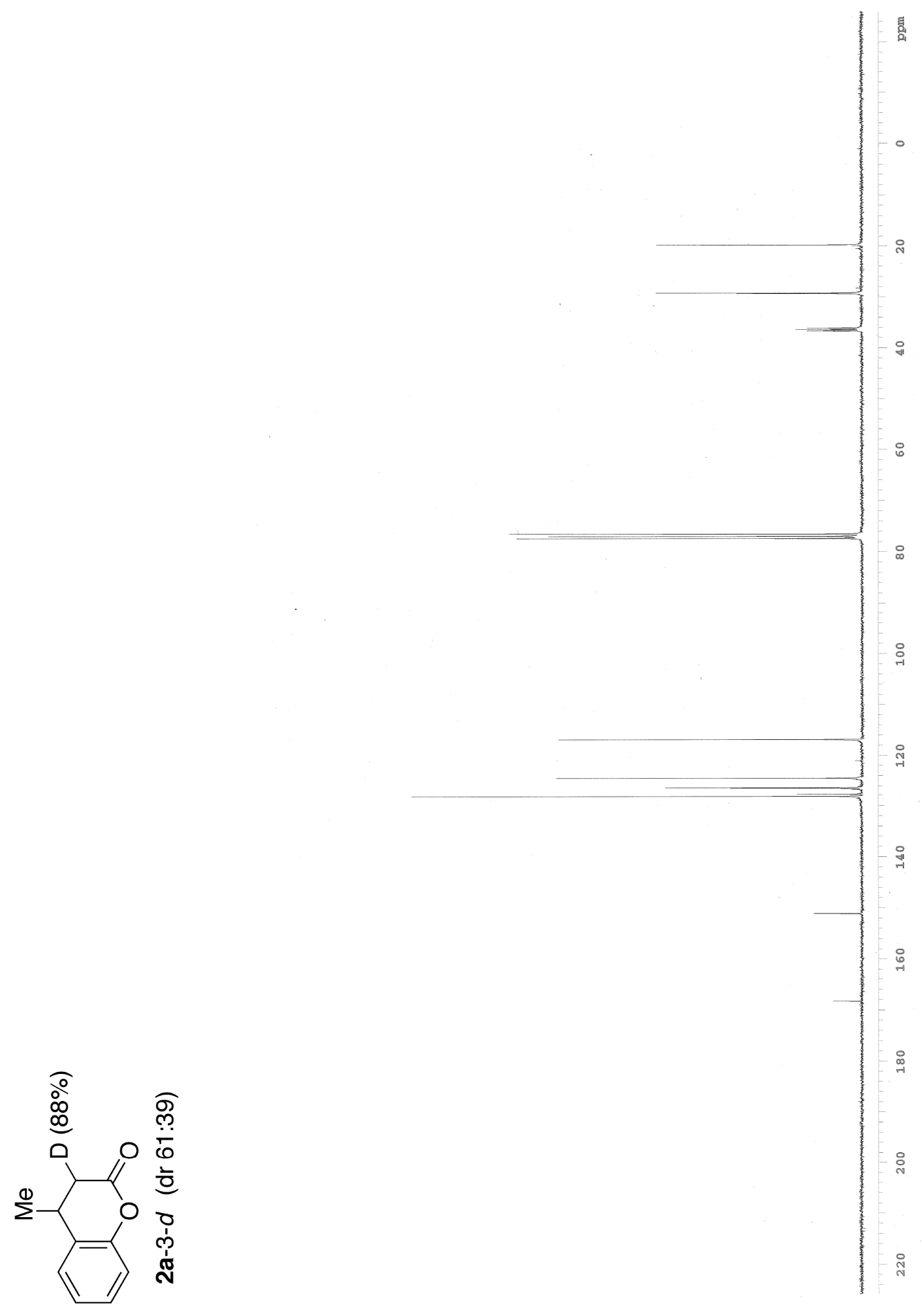




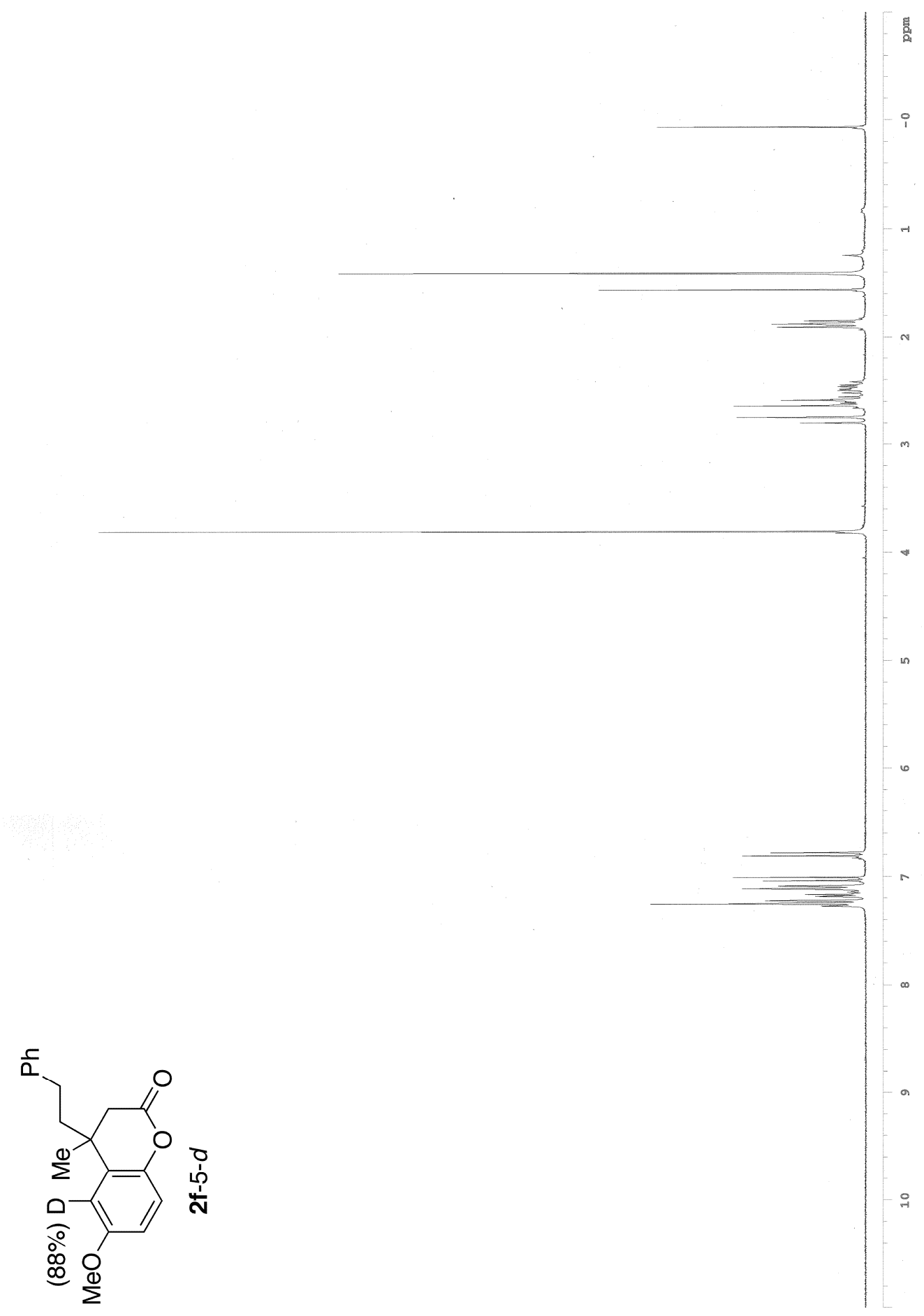




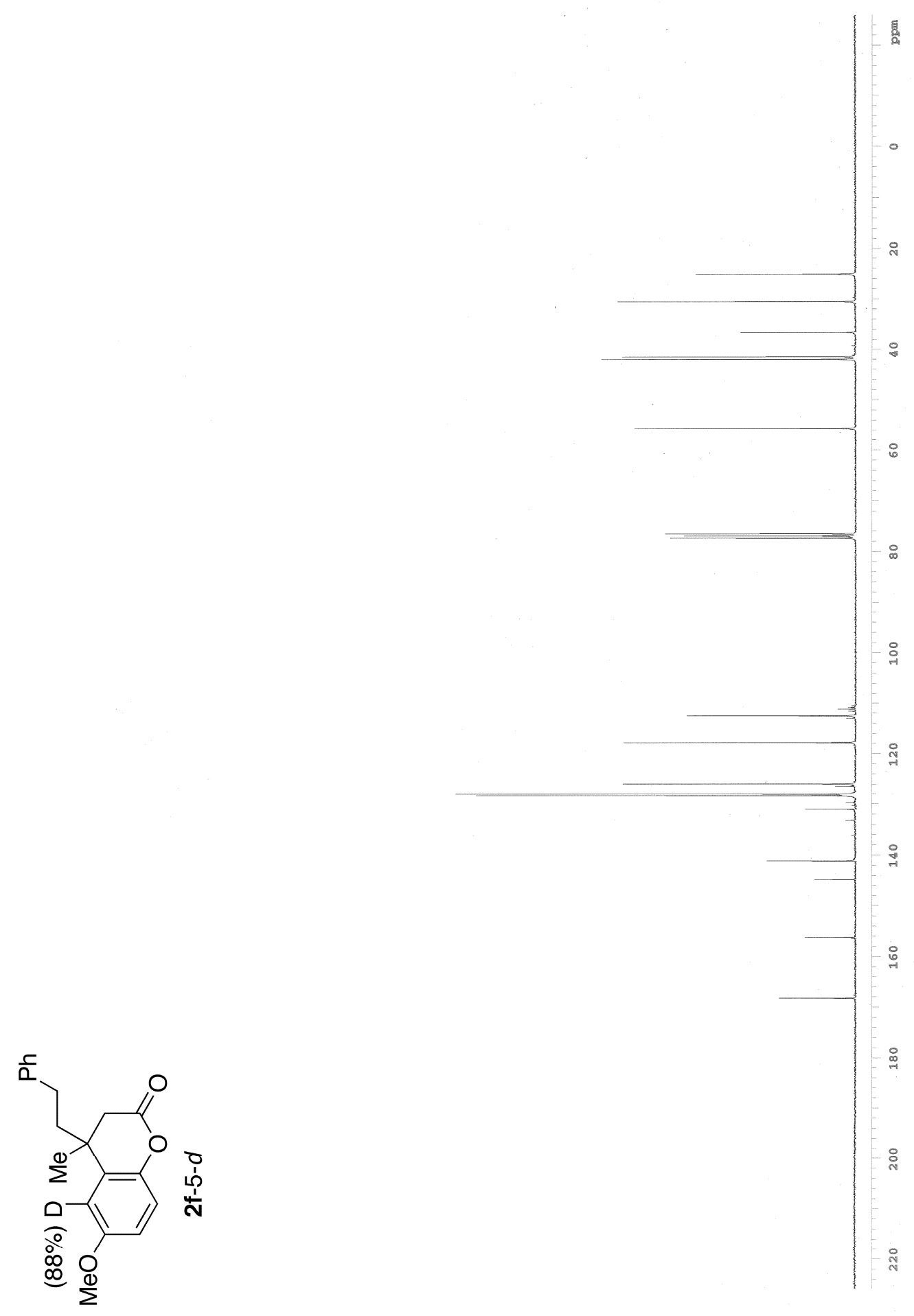

